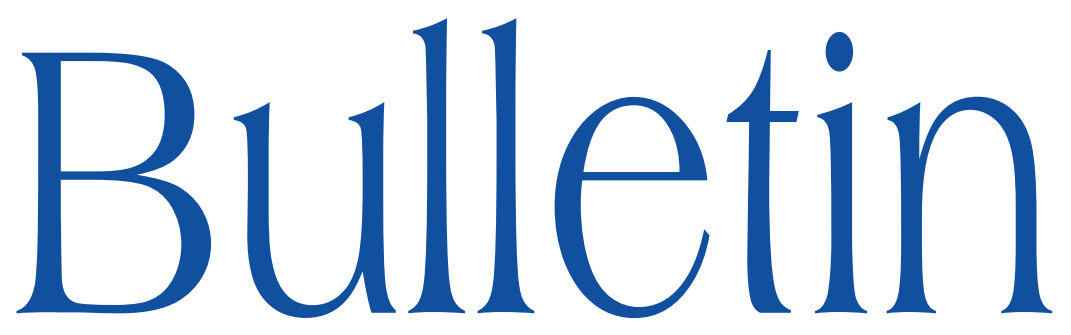

de la SOCIÉTÉ MATHÉMATIQUE DE FRANCE

\title{
SUR LA THÉORIE DE HIDA POUR LE GROUPE GSp $\mathrm{GS}_{2 g}$ \\ Vincent Pilloni
}

\section{Tome 140 Fascicule 3}

2012 


\title{
SUR LA THÉORIE DE HIDA POUR LE GROUPE GSp $\mathrm{GS}_{2 g}$
}

\author{
PAR VINCENT PILLONI
}

\begin{abstract}
RÉsumé. - Nous construisons des familles ordinaires $p$-adiques de formes modulaires pour le groupe $\mathrm{GSp}_{2 g}$. Notre travail généralise et précise des travaux antérieurs de Hida.

Abstract (On Hida theory for the group $\mathrm{GSp}_{2 g}$ ). - We construct ordinary p-adic families of modular forms for the group $\mathrm{GSp}_{2 g}$. Our work generalizes and precises previous work by Hida.
\end{abstract}

\section{Introduction}

Cet article reprend et généralise la théorie des familles $p$-adiques de formes modulaires ordinaires pour le groupe $\mathrm{GSp}_{2 g}$ qui est développée par Haruzo Hida dans [7]. Celle-ci est utilisée par de nombreux auteurs, et nous avons pensé qu'il était utile de jeter une plus grande lumière sur les constructions de loc. cit. afin de préciser certains des énoncés. Ceci nous a permis de généraliser la théorie de Hida pour $\mathrm{GSp}_{2 g}$ et de construire, pour chaque paraboliques standard $\mathrm{P}$ de $\mathrm{GL}_{g}$, une théorie des familles $p$-adiques de formes modulaires de Siegel P-ordinaires. Pour le parabolique de Borel, on retrouve la théorie de [7]. Nous obtenons également des énoncés précis sur la spécialisation des familles de

Texte reçu le 21 septembre 2009, accepté le 28 août 2010.

Vincent Pilloni, Institut Élie Cartan, Université Henri Poincaré, B.P. 239, F-54506 Vandœuvre-les-Nancy Cedex, France

Classification mathématique par sujets (2010). — $11 \mathrm{~F} \mathrm{46,11} \mathrm{G} 18$.

Mots clefs. - Formes modulaires $p$-adiques, variétés de Siegel. 
Hida en des poids dominants. Ces résultats sont utilisés dans [17] pour montrer la classicité de certaines spécialisations en genre 2 .

Nous avons également travaillé avec des structures de niveau plus générales que dans [7], de façon à faciliter les applications arithmétiques de la théorie.

Nous avons de plus inclus un théorème de contrôle horizontal, corollaire facile des techniques développées ici. Nous utilisons ce dernier résultat pour construire des systèmes de Taylor-Wiles dans un autre travail [18].

Enfin, dans l'appendice, nous nous sommes intéressé aux opérateurs $T_{p}$ et $U_{p}$. Nous démontrons de façon géométrique un certains nombre de propriétés fondamentales de ces opérateurs qui sont utilisées dans le texte.

Nous allons maintenant énoncer les résultats principaux pour une structure de niveau principal.

Soit $\mathrm{GL}_{g}$ le groupe algébrique des matrices $g \times g$ inversibles. Soit B le sousgroupe de Borel triangulaire supérieur, $\mathrm{B}^{0}$ le sous-groupe triangulaire inférieur, $\mathrm{T}$ le tore des matrices diagonales et $X(\mathrm{~T})$ son groupe de caractères. Soit $\mathrm{Q}$ un parabolique standard de $\mathrm{GL}_{g}$, triangulaire supérieur par blocs et soit $\mathrm{Q}^{0}$ le parabolique opposé, triangulaire inférieur par bloc. Soit $M_{\mathrm{Q}}$ le sous-groupe de Levi, intersection de $\mathrm{Q}$ et $\mathrm{Q}^{0}$. Notons $X\left(M_{\mathrm{Q}}\right)=X(\mathrm{Q})=X\left(\mathrm{Q}^{0}\right) \subset X(\mathrm{~T})$ le groupe des caractères de $\mathrm{Q}, \mathrm{Q}^{0}$ et $M_{\mathrm{Q}}$. C'est un groupe libre de rang $r$, le nombre de blocs diagonaux de $M_{\mathrm{Q}}$. Fixons un isomorphisme de $\mathbb{Z}^{r}$ avec $X\left(M_{\mathrm{Q}}\right)$ en associant à un $r$-uplet $\left(k_{1}^{\mathrm{Q}}, \ldots, k_{r}^{\mathrm{Q}}\right)$ le caractère $\prod_{i=1}^{r} \operatorname{det}_{i}^{k_{i}^{\mathrm{Q}}}$ où on a posé $\operatorname{det}_{i}$ pour le déterminant du i-ème bloc diagonal en partant du bas. On note $S \mathrm{Q}$ (resp. $S \mathrm{Q}^{0}$ ) le sous-groupe de $\mathrm{Q}$ (resp. $\mathrm{Q}^{0}$ ), intersection des noyaux des caractères de $X(\mathrm{Q})$. Le quotient $\mathrm{Q} / S \mathrm{Q}$ est un tore de caractère $X(\mathrm{Q})$, noté $\mathrm{T}_{\mathrm{Q}}$. On note $X(\mathrm{~T})^{+}$le cône des poids dominants par rapport au sous-groupe de Borel inférieur et $X\left(\mathrm{~T}_{\mathrm{Q}}\right)^{+}=X(\mathrm{~T})^{+} \cap X\left(\mathrm{~T}_{\mathrm{Q}}\right)$ l'ensemble des caractères dominants de $X\left(\mathrm{~T}_{\mathrm{Q}}\right)$. Avec notre identification, $X\left(\mathrm{~T}_{\mathrm{Q}}\right)^{+}$est l'ensemble des $r$-uplets $\left(k_{1}^{\mathrm{Q}}, \ldots, k_{r}^{\mathrm{Q}}\right)$ vérifiant les $r-1$ inégalités $k_{1}^{\mathrm{Q}} \geq \cdots \geq k_{r}^{\mathrm{Q}}$. Un caractère dominant est dit Q-régulier si les inégalités sont strictes : $k_{1}^{\mathrm{Q}}>\cdots>k_{r}^{\mathrm{Q}}$. On dit que le caractère est Q-très régulier si de plus $k_{r}^{\mathrm{Q}} \gg 0$ (Cette dernière terminologie, classique mais un peu ambiguë, est précisée dans la partie 2).

On dispose d'une involution de $X(\mathrm{~T})$,

$$
\kappa=\left(k_{1}, \ldots, k_{g}\right) \mapsto \kappa^{\prime}=\left(-k_{g}, \ldots,-k_{1}\right) .
$$

Cette involution respecte le cône dominant $X(\mathrm{~T})^{+}$. Soit $\omega_{0}$ la matrice antidiagonale de coefficients non nuls égaux à 1 . Notons $\mathrm{P}=\omega_{0}{ }^{t} \mathrm{Q} \omega_{0}$ le parabolique déduit de $\mathrm{Q}$ par conjuguaison par $\omega_{0}$ et transposition. L'involution $\kappa \mapsto \kappa^{\prime}$ échange $X\left(\mathrm{~T}_{\mathrm{P}}\right)$ avec $X\left(\mathrm{~T}_{\mathrm{Q}}\right)$.

Soit $p$ un nombre premier et $\Gamma_{\mathrm{Q}}$ le pro- $p$ sous-groupe maximal de $\mathrm{T}_{\mathrm{Q}}\left(\mathbb{Z}_{p}\right)$. Soit $\mathbb{Z}_{p}\left[\left[\mathrm{~T}_{\mathrm{Q}}\left(\mathbb{Z}_{p}\right)\right]\right]$ et $\Lambda_{\mathrm{Q}}=\mathbb{Z}_{p}\left[\left[\Gamma_{\mathrm{Q}}\right]\right]$ les algèbres de groupe complétées. 
Soit $\bar{X}$ une compactification toroïdale de l'espace de modules sur $\mathbb{Z}_{p}$ des schémas abéliens principalement polarisés, munis d'une structure de niveau $N$ principale avec $N \geq 3$ et $(N, p)=1$ ([3], chap III). Soit $\phi_{\infty}$ le lieu ordinaire de $\bar{X}, \mathcal{J}_{\infty} \rightarrow \phi_{\infty}$ la tour d'Igusa, qui est un pro-revêtement étale galoisien de groupe $\mathrm{GL}_{g}\left(\mathbb{Z}_{p}\right), \mathcal{J}_{\infty, 1}$ le revêtement fini étale de $\phi_{\infty}$, premier cran de la tour d'Igusa et $\mathcal{J}_{\infty, 1} / \mathrm{P}$ le quotient de $\mathcal{J}_{\infty, 1}$ par $\mathrm{P}(\mathbb{Z} / p \mathbb{Z})$. L'algèbre des formes modulaires $p$-adiques pour le parabolique $\mathrm{P}$ est l'algèbre des fonctions régulières sur $\mathcal{T}_{\infty}$, invariantes par le groupe $S \mathrm{P}\left(\mathbb{Z}_{p}\right)$; on la note $V_{\infty}^{S \mathrm{P}}$. On désigne par $V_{\text {cusp }, \infty}^{S \mathrm{P}}$ son idéal cuspidal. Pour tout poids $\kappa \in X\left(\mathrm{~T}_{\mathrm{Q}}\right), V_{\infty}^{S \mathrm{P}}\left[-\kappa^{\prime}\right]$ est le module des formes $p$-adiques pour le parabolique $\mathrm{P}$, de poids $\kappa$ et $V_{\text {cusp }, \infty}^{S \mathrm{P}}\left[-\kappa^{\prime}\right]$ est le sous-module des formes cuspidales. Notons $\omega^{\kappa}$ le faisceau modulaire de poids $\kappa$ sur $\bar{X}$. C'est un faisceau localement libre de $\theta_{\bar{X}}$-modules et ses sections globales forment le module $\mathrm{M}\left(\kappa, \Gamma_{N}, \mathbb{Z}_{p}\right)$ des formes modulaires de Siegel de niveau $N$, poids $\kappa$, à coefficients dans $\mathbb{Z}_{p}$. On note $\mathrm{S}\left(\kappa, \Gamma_{N}, \mathbb{Z}_{p}\right)$ le sous-module des formes cuspidales.

Le théorème suivant est dû à Hida [7] lorsque $\mathrm{P}=\mathrm{Q}$ est le sous-groupe de Borel.

ThÉorème 1.1 (contrôle vertical). - $\quad$ 1. Pour tout poids $\kappa \in X\left(\mathrm{~T}_{\mathrm{Q}}\right)$, il existe un plongement naturel :

$$
\Theta_{\mathrm{P}}^{\star}: \mathrm{M}\left(\kappa, \Gamma_{N}, \mathbb{Z}_{p}\right) \hookrightarrow V_{\infty}^{S \mathrm{P}}\left[-\kappa^{\prime}\right]
$$

2. Il existe un projecteur de $\mathrm{P}$-ordinarité $e_{\mathrm{P}}$ (voir def. 5.1), agissant sur

$$
V_{\infty}^{S \mathrm{P}} \text { et } \mathrm{H}^{0}\left(\mathcal{T}_{\infty, 1} / \mathrm{P}, \omega^{\kappa}\right) \text {. }
$$

3. Pour tout caractère $\kappa \in X\left(\mathrm{~T}_{\mathrm{Q}}\right)$, le $\mathbb{Z}_{p}$-module $e_{\mathrm{P}} V_{\mathrm{cusp}, \infty}^{S \mathrm{P}}\left[-\kappa^{\prime}\right]$ est libre de rang fini.

4. Pour tout poids dominant $\kappa \in X\left(\mathrm{~T}_{\mathrm{Q}}\right)$, on a un isomorphisme :

$$
e_{\mathrm{P}} V_{\infty}^{S \mathrm{P}}\left[-\kappa^{\prime}\right] \simeq e_{\mathrm{P}} \mathrm{H}^{0}\left(\mathcal{J}_{\infty, 1} / \mathrm{P}, \omega^{\kappa}\right)
$$

5. Si le poids $\kappa$ est Q-régulier,

$$
e_{\mathrm{P}} \mathrm{H}^{0}\left(\mathcal{J}_{\infty, 1} / \mathrm{P}, \omega^{\kappa}\right)=e_{\mathrm{P}} \mathrm{H}^{0}\left(\phi_{\infty}, \omega^{\kappa}\right)
$$

6. Si le poids $\kappa$ est $\mathrm{Q}$-très régulier, l'application $\Theta_{\mathrm{P}}^{\star}$ induit un isomorphisme,

$$
e_{\mathrm{P}} \mathrm{S}\left(\kappa, \Gamma_{N}, \mathbb{Z}_{p}\right) \simeq e_{\mathrm{P}} V_{\text {cusp }, \infty}^{S \mathrm{P}}\left[-\kappa^{\prime}\right]
$$

7. Il existe un $\mathbb{Z}_{p}\left[\left[\mathrm{~T}_{\mathrm{Q}}\left(\mathbb{Z}_{p}\right)\right]\right]$-module $V_{\text {cusp }}^{\text {ord } \mathrm{P}, \star}$ qui est libre de rang fini comme $\Lambda_{\mathrm{Q}}$-module et tel que pour tout poids $\kappa \in X\left(\mathrm{~T}_{\mathrm{Q}}\right)$,

$$
V_{\text {cusp }}^{\text {ord-P }, \star} \otimes_{\mathbb{Z}_{p}}\left[\left[\mathrm{~T}_{\mathrm{Q}}\left(\mathbb{Z}_{p}\right)\right]\right], \kappa \mathbb{Z}_{p}=\operatorname{Hom}\left(e_{\mathrm{P}} V_{\text {cusp }, \infty}^{S \mathrm{P}}\left[-\kappa^{\prime}\right], \mathbb{Z}_{p}\right)
$$

8. Lorsque $\mathrm{P}=\mathrm{Q}=\mathrm{GL}_{g}$, les points 6 et 7 valent aussi sans supposer les formes cuspidales. 
Remarques 1.1. - $\quad$ 1. Pour tout sous-groupe parabolique standard P de $\mathrm{GL}_{g}$, de sous-groupe de Levi constitué de $r$ blocs diagonaux, le projecteur $e_{\mathrm{P}}$ est en fait construit à l'aide de $r$ projecteurs associés à $r$ opérateurs de Hecke différents. Cet entier $r$ est la dimension relative sur Spec $\mathbb{Z}_{p}$ de l'espace des poids Spec $\Lambda_{\mathrm{Q}}$. Il y a donc une coïncidence entre le nombre de conditions de contrôle définissant le module des formes modulaires $p$-adiques $\mathrm{P}$-ordinaires et la dimension relative de l'espace des poids.

2. Le projecteur tient compte de la complexité des représentations algébriques du groupe $\mathrm{GL}_{g}$, induites des caractères de $X(\mathrm{P})$, qui fabriquent les faisceaux modulaires $\omega^{\kappa}$ dont les formes modulaires sont les sections. Les caractères de $\mathrm{GL}_{g}$, c'est-à-dire les puissances du déterminant, sont les représentations les plus simples du groupe $\mathrm{GL}_{g}$. Il suffit, dans ce cas des poids « parallèles », d'un seul opérateur de Hecke pour construire le projecteur de $\mathrm{GL}_{g}$-ordinarité. Il y a donc une seule condition de contrôle définissant le module des formes modulaires $p$-adiques $\mathrm{GL}_{g}$-ordinaires.

3. Certains revêtements du lieu ordinaire $\phi_{\infty}$ jouent des rôles particuliers. Sans l'hypothèse de Q-régularité, les formes modulaires $p$-adiques de poids dominant ne proviennent pas de sections du faisceau modulaire $\omega^{\kappa}$ sur le lieu ordinaire $\phi_{\infty}$, mais plutôt de sections définies sur un revêtement $\mathcal{J}_{\infty, 1} / \mathrm{P}$ de celui-ci (voir le point 4 du théorème). Ceci doit donc amener les travaux à venir, notamment sur la théorie des formes modulaires surconvergentes pour le groupe $\mathrm{GSp}_{2 g}$, à considérer ces revêtements plutôt qu'à se restreindre à travailler au niveau de $\phi_{\infty}$. Dans l'appendice, nous démontrons (thm. A.3) que les éléments du module $e_{\mathrm{P}} \mathrm{H}_{\text {cusp }}^{0}\left(\mathcal{J}_{\infty, 1} / \mathrm{P}, \omega^{\kappa}\right)$ surconvergent dans la variété de Siegel de niveau parahorique $\mathrm{P}$.

4. Il est difficile de préciser le point $6 \mathrm{du}$ théorème et de déterminer exactement à quelle condition sur le poids on a un isomorphisme $e_{\mathrm{P}} \mathrm{S}\left(\kappa, \Gamma_{N}, \mathbb{Z}_{p}\right) \simeq e_{\mathrm{P}} V_{\text {cusp }, \infty}^{S \mathrm{P}}\left[-\kappa^{\prime}\right]$. Lorsque $g=1$, cette question est résolue dans [6]. Lorsque $g \geq 2$, le travail de Mokrane et Tilouine [14] répond à cette question sous certaines hypothèses et dans certains cas particuliers. Lorsque $g=2$, nous donnons un critère général dans [17].

Le théorème de contrôle permet de construire des familles de Hida de formes modulaires $p$-adiques, P-ordinaires, cuspidales. Soit $\mathcal{H}^{N p}$ l'algèbre de Hecke sphérique de niveau premier à $N p$. On montre qu'elle agit $\mathbb{Z}_{p}\left[\left[\mathrm{~T}_{\mathrm{Q}}\left(\mathbb{Z}_{p}\right)\right]\right]$-linéairement sur le module $V_{\text {cusp }}^{\text {ord- }, \star}$. La $\mathbb{Z}_{p}\left[\left[\mathrm{~T}_{\mathrm{Q}}\left(\mathbb{Z}_{p}\right)\right]\right]$-sous-algèbre de $\operatorname{End}_{\mathbb{Z}_{p}\left[\left[\mathrm{~T}_{Q}\left(\mathbb{Z}_{p}\right)\right]\right]}\left(\mathcal{V}_{\text {cusp }}^{\text {ord-P }, \star}\right)$ engendrée par l'image de $\mathcal{H}^{N p}$ est notée $\mathbb{T}^{\text {ord-P }}$.

ThÉORÈme 1.2. - Pour toute composante irréductible $C$ de Spec $\mathbb{T}^{\text {ord-P }}$, la projection $f:$ Spec $\mathbb{T}^{\text {ord-P }} \rightarrow$ Spec $\mathbb{Z}_{p}\left[\left[\mathrm{~T}_{\mathrm{Q}}\left(\mathbb{Z}_{p}\right)\right]\right]$ induit un morphisme fini et surjectif $C \rightarrow \operatorname{Spec} \Lambda_{\mathrm{Q}}$.

TOME $140-2012-\mathrm{N}^{\circ} 3$ 
Le morphisme $C \rightarrow$ Spec $\Lambda_{\mathrm{Q}}$ est une famille de Hida. Ses points à valeur dans les extensions finies de $\mathbb{Q}_{p}$ correspondent à des systèmes de valeurs propres de formes modulaires $p$-adiques, P-ordinaires, cuspidales.

Nous allons maintenant donner les grandes lignes du travail.

Après avoir fixé quelques notations, nous étudions dans la partie 3 les représentations du groupe $\mathrm{GL}_{g}$.

Soit $A$ une $\mathbb{Z}_{p}$-algèbre plate, complète pour la toplogie $p$-adique et $K=$ $A[1 / p]$. Pour tout parabolique standard $\mathrm{P}$ de $\mathrm{GL}_{g}$ et tout caractère $\kappa \in X\left(\mathrm{~T}_{\mathrm{P}}\right)^{+}$ vu comme un caractère de $\mathrm{P}^{0}$, nous définissons l'induite algébrique $R_{A}[\kappa]$ de $\mathrm{P}^{0}$ à $\mathrm{GL}_{g}$ (3.1.1); de même, pour tout caractère continu $\kappa$ du groupe de Lie $p$-adique $\mathrm{T}_{\mathrm{P}}\left(\mathbb{Z}_{p}\right)$ étendu à $\mathrm{P}\left(\mathbb{Z}_{p}\right)$, nous définissons des induites toplogiques $\mathcal{F}_{A}^{\mathrm{P}}[\kappa]$ de $\mathrm{P}\left(\mathbb{Z}_{p}\right)$ à $\mathrm{GL}_{g}\left(\mathbb{Z}_{p}\right)$, notées $\mathcal{F}_{A}^{\mathrm{P}}[\kappa]$ (3.1.2). Le paramètre d'induction des induites topologiques est un caractère continu du groupe de Lie $p$-adique $\mathrm{T}_{\mathrm{P}}\left(\mathbb{Z}_{p}\right)$, tandis que le paramètre d'induction des induites algébriques est un caractère algébrique de $T_{P}$. L'application naturelle tordue par l'automorphisme extérieur de $\mathrm{GL}_{g}$,

$$
\mathrm{GL}_{g}\left(\mathbb{Z}_{p}\right) \stackrel{t}{\stackrel{(}{(})^{-1}} \mathrm{GL}_{g} / \mathbb{Z}_{p}
$$

permet de plonger les induites algébriques dans la famille continue des induites topologiques. Nous tentons ensuite de comparer $R_{A}[\kappa]$ et $\mathcal{F}_{A}^{\mathrm{P}}[-\kappa]$ pour un caractère algébrique $\kappa$. Lorsque le parabolique est trivial et vaut $\mathrm{GL}_{g}$, ces deux espaces sont isomorphes : toute fonction homogène de poids $\kappa \in \mathbb{Z}$ sur $\mathbb{Z}_{p}^{\times}$ est algébrique! En générale, l'induite topologique est beaucoup plus grosse que l'induite algébrique. Nous introduisons alors des projecteurs $e_{\mathrm{P}}^{\text {top }}$ et $e_{\mathrm{P}}^{\text {alg }}$ sur les induites (3.2). Ces projecteurs ne commutent pas à l'action du groupe, ils commutent seulement à l'action des tores $\mathrm{T}_{\mathrm{P}}$ et $\mathrm{T}_{\mathrm{P}}\left(\mathbb{Z}_{p}\right)$ et ils ont pour effet de ramener l'étude des fonctions sur $\mathrm{GL}_{g}$ ou $\mathrm{GL}_{g}\left(\mathbb{Z}_{p}\right)$, induites d'un caractère de $\mathrm{P}^{0}$ ou $\mathrm{P}\left(\mathbb{Z}_{p}\right)$, à celle des fonctions sur les tores $\mathrm{T}_{\mathrm{P}}$ et $\mathrm{T}_{\mathrm{P}}\left(\mathbb{Z}_{p}\right)$. Lorsque le poids est dominant, $e_{\mathrm{P}}^{\text {alg }}$ projette l'induite algébrique sur un espace de dimension 1. De même, lorsque le poids est P-régulier, $e_{\mathrm{P}}^{\text {top }}$ projette l'induite topologique sur un espace de dimension 1.

Après ces préliminaires sur les représentations du groupe $\mathrm{GL}_{g}$, nous introduisons, dans la partie 4, le module des formes modulaires et le module des formes modulaires $p$-adiques. On peut comparer ces deux espaces. La comparaison repose sur le fait suivant : si $\theta$ est l'anneau des entiers d'une extension finie de $\mathbb{Q}_{p}$ et $\mathscr{G} \rightarrow$ Spec $\Theta$ est un schéma abélien ordinaire de section unité $e$, une trivialisation de la partie connexe $\mathscr{C}\left[p^{\infty}\right]^{0}$ du groupe de Barsotti-Tate associé à $\mathscr{C}$ induit une trivialisation du faisceau conormal $e^{\star} \Omega_{\mathscr{Q} / \vartheta}^{1}$ via l'application de Hodge-Tate. Ceci permet de plonger, sur le lieu ordinaire, les fonctions sur le $\mathrm{GL}_{g}$-torseur des trivialisations du faisceau conormal dans l'ensemble des 
fonctions sur le $\mathrm{GL}_{g}\left(\mathbb{Z}_{p}\right)$-torseur d'Igusa. On obtient alors naturellement, pour tout poids $\kappa \in X\left(\mathrm{~T}_{\mathrm{Q}}\right)$, une suite de morphismes (4.2.1):

$$
\mathrm{H}^{0}\left(\bar{X}, \omega^{\kappa}\right) \stackrel{i_{1}}{\hookrightarrow} \mathrm{H}^{0}\left(\phi_{\infty}, \omega^{\kappa}\right) \stackrel{i_{2}}{\hookrightarrow} V_{\infty}^{S \mathrm{P}}\left[-\kappa^{\prime}\right]
$$

L'application $i_{1}$ est simplement une restriction, l'application $i_{2}$ est localement le plongement de l'induite algébrique dans l'induite topologique.

L'avantage du module $V_{\infty}^{S P}$ est de «mettre ensemble » des formes de poids variables et de permettre l'étude des congruences entre formes de poids différents. Mais c'est un espace gigantesque. Nous allons le projeter, suivant l'idée désormais classique de Hida, sur un sous-module sur lequel, pour un ensemble Zariski-dense de poids, l'application $i_{2} \circ i_{1}$ est un isomorphisme.

Nous définissons donc, au numéro 5 , le projecteur de P-ordinarité. Il joue un double rôle. Localement sur $\phi_{\infty}$, il agit sur $V_{\infty}^{S \mathrm{P}}\left[-\kappa^{\prime}\right]$ et $\operatorname{sur} \mathrm{H}^{0}\left(\phi_{\infty}, \omega^{\kappa}\right)$ comme les projecteurs $e_{\mathrm{P}}^{\text {alg }}$ et $e_{\mathrm{P}}^{\text {top }}$ étudiés dans la partie 3. Il sélectionne des vecteurs algébriques dans l'induite topologique $V_{\infty}^{S \mathrm{P}}\left[-\kappa^{\prime}\right]$. Précisément, lorsque le poids est Q-régulier, on montre que $i_{2}$ devient un isomorphisme après la projection. D'autre part, le projecteur contrôle l'obstruction au prolongement des formes définies sur le lieu ordinaire en des formes définies partout (il contrôle l'ordre des pôles sur le complémentaire du lieu ordinaire). Lorsque le poids est Q-très régulier (et les formes sont de plus cuspidales lorsque $\mathrm{Q} \neq \mathrm{GL}_{g}$ ), $i_{1}$ devient un isomorphisme après la projection. La vérification des propriétés $d u$ projecteur (5.2) est assez délicate. Pour montrer que $i_{1}$ réalise un isomorphisme sur la partie $\mathrm{P}$-ordinaire lorsque le poids est Q-très régulier, nous utilisons les résultats de l'appendice (thm. A.1).

Le numéro 6 résout un certain nombre de difficultés techniques liées au problème du relèvement des formes modulaires et des formes $p$-adiques, de la caractéristique $p$ vers la caractéristique zéro (6.1). La condition de cuspidalité est cruciale dans le cas des poids non parallèles. Il existe une obstruction à l'existence d'une bonne théorie pour des poids non parallèles et des formes non cuspidales.

Dans la partie 7, nous établissons le théorème de contrôle vertical (thm. 7.1 et 7.2). On en déduit ensuite l'existence des familles de Hida (thm. 8.1).

La dernière partie est dédiée au théorème de contrôle horizontal (thm. 9.1) qui est utilisé dans [18].

Dans l'appendice, on démontre des théorèmes de finitude pour la partie $U_{p}$-ordinaire des modules $\mathrm{H}_{\text {cusp }}^{0}\left(\phi_{\infty}, \omega^{\kappa}\right)$. On montre la surconvergence des éléments $U_{p}$-ordinaires cuspidaux et on établit que des section $U_{p}$-ordinaires, cuspidales, pour un poids $\kappa=\left(k_{1}, \ldots, k_{g}\right)$ avec $k_{g} \gg 0$ sont des formes modulaires de Siegel classiques (thm. A.1, thm. A.3).

TOME $140-2012-\mathrm{N}^{\mathrm{O}} 3$ 
Remerciements. - Je tiens à remercier Jacques Tilouine pour ses conseils et ses encouragements tout au long de l'élaboration de ce travail.

\section{Notations}

Soit $g$ un entier supérieur ou égal à 2. Soit $\mathrm{GL}_{g}$ le groupe linéaire de dimension $g$ sur $\mathbb{Z}$ qu'on réalise comme le groupe des matrices $g \times g$ inversibles, $\mathrm{SL}_{g} \subset \mathrm{GL}_{g}$ le groupe spécial, $\mathrm{B} \subset \mathrm{GL}_{g}$ le sous-groupe de Borel triangulaire supérieur et $\mathrm{B}^{0}$ le Borel opposé des matrices triangulaires inférieures. On notera ${ }^{t}: \mathrm{GL}_{g} \rightarrow \mathrm{GL}_{g}$ la transposition qui échange $\mathrm{B}$ et $\mathrm{B}^{0}$.

Soit $\mathrm{T}=\mathrm{B} \cap \mathrm{B}^{0}$ le tore maximal associé et $\mathrm{U}$ (resp. $\mathrm{U}^{0}$ ) le radical unipotent de $\mathrm{B}\left(\right.$ resp. $\left.\mathrm{B}^{0}\right)$. On note $X(\mathrm{~T})$ le groupe des caractères de $\mathrm{T}$ et $X(\mathrm{~T})^{+}$le cône des poids dominants par rapport à $\mathrm{B}^{0}$. On identifie $X(\mathrm{~T})$ à $\mathbb{Z}^{g}$ en associant à tout $g$-uplets $\left(k_{1}, k_{2}, \ldots, k_{g}\right)$ le caractère $\operatorname{diag}\left(t_{g}, t_{g-1}, \ldots, t_{1}\right) \mapsto \prod_{i} t_{i}^{k_{i}}$. Avec cette convention $X(\mathrm{~T})^{+}$est l'ensemble des $g$-uplets $\left(k_{1}, k_{2}, \ldots, k_{g}\right)$ tels que $k_{1} \geq k_{2} \geq \cdots \geq k_{g}$. On a une involution $\kappa \mapsto \kappa^{\prime}$ qui à $\left(k_{1}, k_{2}, \ldots, k_{g}\right)$ associe $\left(-k_{g},-k_{g-1}, \ldots,-k_{1}\right)$ et qui respecte le cône dominant.

Soit $\left(n_{1}, \ldots, n_{r}\right)$ des entiers strictements positifs tels que $\sum_{i=1}^{r} n_{i}=g$. On pose, pour $1 \leq l \leq r, N_{l}=\sum_{i=1}^{l} n_{i}$. Soit $\mathrm{P}$ le parabolique standard de $\mathrm{GL}_{g}$ des matrices triangulaires supérieures par blocs associé aux entiers $\left(n_{i}\right)_{1 \leq i \leq r}$,

$$
\mathrm{P}=\left\{M \in \mathrm{GL}_{g}, \quad M=\left(\begin{array}{cccc}
M_{r} & & \star & \star \\
\ddots & & \\
0 & M_{i} & \star \\
& & \ddots & \\
0 & 0 & M_{1}
\end{array}\right), M_{i} \in \mathrm{GL}_{n_{i}}\right\}
$$

et aussi $S \mathrm{P}$ le sous-groupe spécial de $\mathrm{P}$ défini par

$$
S \mathrm{P}=\left\{M \in \mathrm{GL}_{g}, \quad M=\left(\begin{array}{cccc}
M_{r} & & \star & \star \\
\ddots & & \\
0 & M_{i} & \star \\
0 & 0 & M_{1}
\end{array}\right), M_{i} \in \mathrm{SL}_{n_{i}}\right\} .
$$

Le quotient $\mathrm{T}_{\mathrm{P}}=\mathrm{P} / S \mathrm{P}$ est un tore de dimension $r$ appelé parfois cocentre de $\mathrm{P}$. On notera aussi $S \mathrm{P}^{0} \subset \mathrm{P}^{0}$ le parabolique opposé et son sous-groupe spécial.

Soit $X\left(\mathrm{P}^{0}\right)=X(\mathrm{P})=X\left(\mathrm{~T}_{\mathrm{P}}\right)$ le groupe des caractères de $\mathrm{P}^{0}, \mathrm{P}$ et $\mathrm{T}_{\mathrm{P}}$. C'est un groupe libre de rang $r$ qu'on identifie à $\mathbb{Z}^{r}$ en associant au $r$-uplet $\left(k_{1}^{\mathrm{P}}, \ldots, k_{r}^{\mathrm{P}}\right)$ le caractère $M \mapsto \prod_{i=1}^{r}\left(\operatorname{det} M_{i}\right)^{k_{i}^{\mathrm{P}}}$.

Le plongement naturel $X\left(\mathrm{~T}_{\mathrm{P}}\right) \hookrightarrow X(\mathrm{~T})$ est donné, avec nos identifications, par l'application $\mathbb{Z}^{r} \hookrightarrow \mathbb{Z}^{g}$ qui au $r$-uplet $\left(k_{1}^{\mathrm{P}}, \ldots, k_{r}^{\mathrm{P}}\right)$ associe le $g$-uplets $\left(k_{1}, \ldots, k_{g}\right)$ défini par $k_{1}=\cdots=k_{n_{1}}=k_{1}^{\mathrm{P}}, k_{n_{1}+1}=\cdots=k_{n_{1}+n_{2}}=k_{2}^{\mathrm{P}}, \ldots$. Soit $X\left(\mathrm{~T}_{\mathrm{P}}\right)^{+}=X\left(\mathrm{~T}_{\mathrm{P}}\right) \cap X(\mathrm{~T})^{+}$le cône des caractères dominants. 
Pour tout parabolique standard $\mathrm{P}$, on dit qu'un poids $\kappa=\left(k_{1}^{\mathrm{P}}, \ldots, k_{r}^{\mathrm{P}}\right) \in$ $X\left(\mathrm{~T}_{\mathrm{P}}\right)$ est P-régulier si $k_{1}^{\mathrm{P}}>k_{2}^{\mathrm{P}}>\cdots>k_{r}^{\mathrm{P}}$. Un tel poids est donc dominant. On note $X\left(\mathrm{~T}_{\mathrm{P}}\right)^{++}$le cône des caractères P-réguliers. Pour tout entier $t \in \mathbb{Z}$, on note $\underline{t} \in \mathbb{Z}^{g}$ le $g$-uplet parallèle de valeur $t$. Pour tout poids P-régulier $\kappa$, soit la droite $D_{\kappa}=\{\kappa+\underline{t}, t \in \mathbb{Z}\} \subset X\left(\mathrm{~T}_{\mathrm{P}}\right)^{++}$. On suppose donné, pour chaque droite $D_{\kappa}$, un entier $N\left(D_{\kappa}\right)$ induisant une demi-droite $D_{\kappa}^{+}=\left\{\kappa+\underline{t}, t \geq N\left(D_{\kappa}\right)\right\}$. On dit alors qu'un poids P-régulier $\kappa$ est P-très régulier si $\kappa \in D_{\kappa}^{+}$. Lorsque nous utiliserons cette notion, les entiers $N\left(D_{\kappa}\right)$ seront non explicites et sousentendus. On abrègera fréquemment en disant qu'un poids $\kappa$ est $\mathrm{P}$-très régulier si il est régulier et de plus $k_{r}^{\mathrm{P}} \gg 0$.

Soit $\omega_{0} \in \mathrm{GL}_{g}(\mathbb{Z})$ la matrice antidiagonale de coefficients non nuls égaux à 1. Si $\mathrm{P}$ est un parabolique standard de $\mathrm{GL}_{g}$ associé aux entiers $\left(n_{i}\right)_{1 \leq i \leq r}$, $\omega_{0} \cdot{ }^{t} \mathrm{P} \cdot \omega_{0}=\mathrm{Q}$ est le parabolique standard de $\mathrm{GL}_{g}$ associé aux entiers $\left(n_{r+1-i}\right)_{1 \leq i \leq r}$. L'involution ' $: X(\mathrm{~T}) \rightarrow X(\mathrm{~T})$ échange $X\left(\mathrm{~T}_{\mathrm{P}}\right)$ et $X\left(\mathrm{~T}_{\mathrm{Q}}\right)$, $X\left(\mathrm{~T}_{\mathrm{P}}\right)^{+}$et $X\left(\mathrm{~T}_{\mathrm{Q}}\right)^{+}$ainsi que $X\left(\mathrm{~T}_{\mathrm{P}}\right)^{++}$et $X\left(\mathrm{~T}_{\mathrm{Q}}\right)^{++}$.

On note $W$ le groupe de Weyl de $\mathrm{GL}_{g}$ et pour tout parabolique standard $\mathrm{P}$ de sous-groupe de Levi standard $\mathrm{M}$, soit $W_{\mathrm{M}} \subset W$ le groupe de Weyl de $\mathrm{M}$. $\nu \in \mathbb{G}_{m}$.

On note $\mathrm{G}=\mathrm{GSp}_{2 \mathrm{~g}}=\left\{X \in \mathrm{GL}_{2 g},{ }^{t} X J X=\nu . J\right\}$ avec $J=\left(\begin{array}{cc}0 & \omega_{0} \\ -\omega_{0} & 0\end{array}\right)$ et

Soit $\mathrm{B}_{\mathrm{G}}$ le sous-groupe de Borel des matrices triangulaires supérieures, $\mathrm{T}_{\mathrm{G}}$ le tore maximal standard contenu dans $\mathrm{B}_{\mathrm{G}}$ et $\mathrm{U}_{\mathrm{G}}$ le radical unipotent.

On dispose d'un plongement de $\mathrm{GL}_{g} \hookrightarrow \mathrm{G}$ qui envoie $A \in \mathrm{GL}_{g}$ sur $\left(\begin{array}{cc}A & 0 \\ 0 & \omega_{0}{ }^{t} A^{-1} \omega_{0}\end{array}\right)$.

On introduit le parabolique de Siegel, $\mathrm{S}=\left\{\left(\begin{array}{cc}A & \star \\ 0 & \nu . \omega_{0}{ }^{t} A^{-1} \omega_{0}\end{array}\right) \in \mathrm{G} ; A \in\right.$ $\left.\mathrm{GL}_{g}, \nu \in \mathbb{G}_{m}\right\}$.

Pour tout parabolique standard $\mathrm{P}$ de $\mathrm{GL}_{g}$ on peut également considérer le parabolique

$$
\mathrm{S}_{\mathrm{P}}=\left\{\left(\begin{array}{lc}
A & \star \\
0 & \nu \cdot \omega_{0}{ }^{t} A^{-1} \omega_{0}
\end{array}\right) \in \mathrm{G} ; A \in \mathrm{P}, \nu \in \mathbb{G}_{m}\right\}
$$

et son sous-groupe $\mathrm{S}_{S \mathrm{P}}=\left\{\left(\begin{array}{cc}A & \star \\ 0 & \nu \cdot \omega_{0}{ }^{t} A^{-1} \omega_{0}\end{array}\right) \in \mathrm{G} ; A \in S \mathrm{P}, \nu \in \mathbb{G}_{m}\right\}$.

TOME $140-2012-\mathrm{N}^{\mathrm{O}} 3$ 
On introduit aussi le parabolique de Klingen $\mathrm{K}=\left\{\left(\begin{array}{cc}\star & \star \\ 0 & A \star \\ 0 & 0 \star\end{array}\right) \in \mathrm{G} ; A \in\right.$ $\left.\mathrm{GSp}_{2(g-1)}\right\}$ et son sous-groupe, dit parabolique de Klingen strict :

$$
\mathrm{K}^{+}=\left\{\left(\begin{array}{cc}
1 & \star \star \\
0 & A \star \\
0 & 0
\end{array}\right) \in \mathrm{G} ; A \in \mathrm{GSp}_{2(g-1)}\right\}
$$

2.0.0.1. Convention. - Pour tout groupe $H$ et tout module $M$ muni d'une action de $H$, nous notons $M^{H}$ le sous-module des invariants sous $H$. Si $\kappa$ est un caractère de $H$, nous notons $M[\kappa]$ le sous-module de $M$ des éléments $\kappa$-variants sous l'action de $H$.

Si $H$ est un groupe algébrique et $A$ un anneau tel que $H(A)$ agit sur $M$, on notera $M^{H}=M^{H(A)}$ si cela ne prête pas à confusion. De même si $Z$ est un schéma équipé d'une action de $H(A)$ et si le quotient géométrique $Z /(H(A))$ existe, on le notera parfois $Z / H$.

\section{Représentations de $\mathrm{GL}_{g}$}

\subsection{Induction}

3.1.1. Induction algébrique. - Soit $A$ un anneau commutatif. On définit le A-module :

$$
R_{A}=\left\{f: \mathrm{GL}_{g} /{ }_{A} \rightarrow \mathbb{A}^{1} / A, f(g u)=f(g) \forall u \in \mathrm{U}^{0}\right\} .
$$

Le groupe $\mathrm{T}$ agit par translation à droite sur $\mathrm{GL}_{g}$ et induit une action sur $R_{A}$. On a la décomposition :

$$
R_{A}=\oplus_{\kappa \in X(\mathrm{~T})}+R_{A}[\kappa]
$$

où $R_{A}[\kappa]=\left\{f: \mathrm{GL}_{g} /_{A} \rightarrow \mathbb{A}^{1} / A, f(g u)=f(g) \forall u \in \mathrm{U}^{0}\right.$ et $f(g t)=$ $\kappa(t) f(g) \forall t \in T\}$. Ces modules sont libres de rang fini sur $A$.

$\mathrm{Si}$ on fait agir $\mathrm{GL}_{g}$ à gauche sur $R_{A}[\kappa]$ par $g . f=f\left(g^{-1}.\right)$ on trouve que $R_{A}[\kappa]^{U}$ est la droite de poids $\kappa^{-1}$ et que $R_{A}[\kappa]^{U^{0}}$ est la droite de poids $\kappa^{\prime}$ qui est un élément de $X(\mathrm{~T})^{+}$.

Pour tout $\kappa$, on dispose de formes linéaires $\mathrm{B}^{0}$-équivariante :

$$
\begin{aligned}
\ell_{c a n}: R_{A}[\kappa] & \rightarrow A\left(\kappa^{-1}\right) \\
f & \mapsto f(1)
\end{aligned}
$$

où $\mathrm{B}^{0}$ agit sur $R_{A}$ par l'action précédente, induite par la translation à gauche sur $\mathrm{GL}_{g}$, et $\operatorname{sur} A$ via $\kappa^{-1}$. 
Soit $\mathrm{P}$ un parabolique standard de $\mathrm{GL}_{g}$ associé à des entiers $\left(n_{i}\right)_{1 \leq i \leq r}$. L'induite

$$
R_{A}^{\mathrm{P}}=\left\{f: \mathrm{GL}_{g} /{ }_{A} \rightarrow \mathbb{A}^{1} / A, f(g u)=f(g) \forall u \in S \mathrm{P}^{0}\right\}
$$

est un sous-module de $R_{A}$. L'action du tore $\mathrm{T}$ (induite par translation à droite sur $\left.\mathrm{GL}_{g}\right)$ sur $R_{A}$ respecte le sous-module $R_{A}^{\mathrm{P}}$. L'action de $\mathrm{T}$ sur $R_{A}^{\mathrm{P}}$ se factorise par $\mathrm{T}_{\mathrm{P}}$.

Si $\kappa \in X\left(\mathrm{~T}_{\mathrm{P}}\right)^{+}$, on a en fait $R_{A}[\kappa]=\left\{f: \mathrm{GL}_{g} /{ }_{A} \rightarrow \mathbb{A}^{1} / A, f(g p)=\right.$ $\left.\kappa(p) f(g) \forall p \in \mathrm{P}^{0}\right\}$ et donc

$$
R_{A}^{\mathrm{P}}=\oplus_{\kappa \in X\left(\mathrm{~T}_{\mathrm{P}}\right)}+R_{A}[\kappa] .
$$

On a une application de restriction $\mathrm{T}_{\mathrm{P}}$-équivariante :

$$
\begin{aligned}
\text { res }: R_{A}^{\mathrm{P}} & \rightarrow \operatorname{Hom}\left(\mathrm{P}^{0} / S \mathrm{P}^{0}, \mathbb{A}^{1}\right) \\
f & \left.\mapsto f\right|_{\mathrm{P}^{0}}
\end{aligned}
$$

Notons $R_{A}^{0}$ le noyau de l'application res. Pour tout poids $\kappa \in X\left(\mathrm{~T}_{\mathrm{P}}\right)^{+}$, l'application res : $R_{A}[\kappa] \rightarrow A$. $\kappa$ est surjective. On dispose alors d'une suite exacte de $\mathrm{T}_{\mathrm{P}}$-modules :

$$
0 \rightarrow R_{A}^{0}[\kappa] \rightarrow R_{A}[\kappa] \rightarrow A . \kappa \rightarrow 0
$$

Remarque 3.1. - Dans la notation de Jantzen ([9], I 3.3) $R_{A}[\kappa]=$ $\operatorname{Ind}_{\mathrm{B}^{0}}^{\mathrm{GL}_{g}} \kappa^{-1}$ et $\kappa^{-1}$ est dominant par rapport à $\mathrm{B}$.

3.1.2. Induction topologique. - Soit $A$ une $\mathbb{Z}_{p}$-algèbre plate, complète pour la topologie $p$-adique et $K=A[1 / p]$. Soit $\mathrm{P}$ un parabolique standard de $\mathrm{GL}_{g}$ et $\kappa \in X\left(\mathrm{~T}_{\mathrm{P}}\right)$ un caractère. Notons $\mathcal{F}_{K}^{\mathrm{P}}[\kappa]$ le $K$-module des fonctions continues $f$ : $\mathrm{GL}_{g}\left(\mathbb{Q}_{p}\right) \rightarrow K$ telles que $f(g p)=\kappa(p) f(g)$ pour tout $g \in \mathrm{G}\left(\mathbb{Q}_{p}\right)$ et $p \in \mathrm{P}\left(\mathbb{Q}_{p}\right)$. Remarquons qu'une fonction $f \in \mathcal{F}_{K}^{\mathrm{P}}[\kappa]$ est déterminée par sa restriction à $\mathrm{GL}_{g}\left(\mathbb{Z}_{p}\right)$ et que toute fonction continue $f: \mathrm{GL}_{g}\left(\mathbb{Z}_{p}\right) \rightarrow K$ vérifiant l'équation fonctionnelle $f(g p)=\kappa(p) f(g)$ pour tout $g \in \mathrm{GL}_{g}\left(\mathbb{Z}_{p}\right)$ et $p \in \mathrm{P}\left(\mathbb{Z}_{p}\right)$ se prolonge uniquement en un élément de $\mathcal{F}_{K}^{\mathrm{P}}[\kappa]$. Soit $\mathcal{F}_{A}^{\mathrm{P}}[\kappa]$ le sous $A$-module de $\mathcal{F}_{K}^{\mathrm{P}}[\kappa]$ des fonctions $f: \mathrm{GL}_{g}\left(\mathbb{Z}_{p}\right) \rightarrow A$ qui vérifient $f(g p)=\kappa(p) f(g)$ pour tout $g \in \mathrm{GL}_{g}\left(\mathbb{Z}_{p}\right)$ et $p \in \mathrm{P}\left(\mathbb{Z}_{p}\right)$.

On note

$$
\text { res : } \mathscr{F}_{K}^{\mathrm{P}}[\kappa] \rightarrow K . \kappa
$$

la restriction des fonctions à $\mathrm{P}$ et on note $\mathcal{F}_{K}^{\mathrm{P}, 0}[\kappa]$ le noyau de l'application res. On note aussi $\mathcal{F}_{A}^{\mathrm{P}, 0}=\mathcal{F}_{K}^{\mathrm{P}, 0}[\kappa] \cap \mathcal{F}_{A}^{\mathrm{P}}[\kappa]$. On dispose donc d'une suite exacte de $\mathrm{T}_{\mathrm{P}}\left(\mathbb{Z}_{p}\right)$-modules :

$$
0 \rightarrow \mathcal{F}_{A}^{\mathrm{P}, 0}[\kappa] \rightarrow \mathcal{F}_{A}^{\mathrm{P}}[\kappa] \rightarrow A . \kappa \rightarrow 0 .
$$

On a un plongement naturel de schémas en groupe $\mathrm{GL}_{g}\left(\mathbb{Z}_{p}\right) \hookrightarrow \mathrm{GL}_{g} / \mathbb{Z}_{p}$. Pour des raisons qui apparaîtrons claires par la suite, on est amené à tordre cette 
injection par l'automorphisme extérieur ${ }^{t}()^{-1}$ de $\mathrm{GL}_{g}$. On a une application injective

$$
\begin{aligned}
i: R_{K}[\kappa] & \rightarrow \mathcal{F}_{K}^{\mathrm{P}}[-\kappa] \\
f & \mapsto\left[\mathrm{GL}_{g}\left(\mathbb{Q}_{p}\right) \stackrel{{ }^{t}()^{-1}}{\hookrightarrow} \mathrm{GL}_{g}(K) \stackrel{f}{\rightarrow} \mathbb{A}^{1}(K)\right] .
\end{aligned}
$$

On note $\tilde{R}_{A}[\kappa]=i^{-1}\left(\mathcal{F}_{A}^{\mathrm{P}}[-\kappa]\right)$, c'est un réseau de $R_{K}[\kappa]$ contenant $R_{A}[\kappa]$. On a enfin un diagramme commutatif

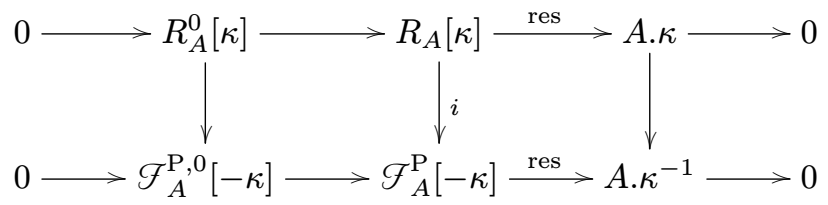

3.2. Projecteur d'ordinarité. - Pour $j=0, \ldots, g-1$, nous posons $\alpha_{j}=$ $\left(\begin{array}{cc}1_{g-j} & 0 \\ 0 & p .1_{j}\end{array}\right) \in \mathrm{GL}_{g}\left(\mathbb{Q}_{p}\right)$. Notons $\mathrm{T}^{+}\left(\mathbb{Q}_{p}\right)$ le sous-monoïde de $\mathrm{T}\left(\mathbb{Q}_{p}\right)$ engendré par les matrices $\left\{\alpha_{j}, j=0, \ldots, g-1\right\}$. Soit $\mathrm{P}$ un parabolique standard de $\mathrm{GL}_{g}$ et $\kappa \in X\left(\mathrm{~T}_{\mathrm{P}}\right)$ un caractère. Soit $A$ une $\mathbb{Z}_{p}$ algèbre plate, complète pour la topologie $p$-adique et $K=A[1 / p]$. Pour tout $\alpha \in \mathrm{T}^{+}\left(\mathbb{Q}_{p}\right)$, définissons les opérateurs :

$$
\begin{aligned}
t^{\mathrm{alg}}(\alpha): R_{K}[\kappa] & \rightarrow R_{K}[\kappa] \\
f & \mapsto f\left(\alpha^{-1} \cdot \alpha\right) \\
t^{\mathrm{top}}(\alpha): \mathcal{F}_{K}^{\mathrm{P}}[-\kappa] & \rightarrow \mathcal{F}_{K}^{\mathrm{P}}[-\kappa] \\
f & \mapsto f\left(\alpha \cdot \alpha^{-1}\right)
\end{aligned}
$$

Les opérateurs $t^{\text {alg }}(\alpha)$ et $t^{\text {top }}(\alpha)$ sont compatibles avec le morphisme $R_{K}[\kappa] \rightarrow$ $\mathcal{F}_{K}^{\mathrm{P}}[-\kappa]$. Lorsque le contexte est clair, on note seulement $t(\alpha)$ au lieu de $t^{\text {top }}(\alpha)$ ou $t^{\operatorname{alg}}(\alpha)$.

Proposition 3.1. - Supposons que $\kappa \in X\left(\mathrm{~T}_{\mathrm{P}}\right)^{+}$. L' opérateur $t^{\text {alg }}(\alpha)$ stabilise le réseau $\tilde{R}_{A}[\kappa]$ de $R_{K}[\kappa]$. L'opérateur $t^{\mathrm{top}}(\alpha)$ stabilise le sous-module $\mathcal{F}_{A}^{\mathrm{P}}[-\kappa]$ de $\mathcal{F}_{K}^{\mathrm{P}}[-\kappa]$.

Démonstration. - Il suffit de vérifier cet énoncé pour les opérateurs $t\left(\alpha_{j}\right)=t_{j}$. Rappelons la décomposition d'Iwahori :

$$
\mathrm{GL}_{g}\left(\mathbb{Z}_{p}\right)=\coprod_{w \in W} \mathrm{I} w \mathrm{U}^{0}\left(\mathbb{Z}_{p}\right)
$$


où I est le groupe d'Iwahori des matrices triangulaires supérieures modulo $p$. Soit $i . w$ un élément de $\mathrm{GL}_{g}\left(\mathbb{Z}_{p}\right) \bmod \mathrm{U}^{0}\left(\mathbb{Z}_{p}\right)$ et $f \in \tilde{R}_{A}[\kappa]$, on a donc

$$
\begin{aligned}
t_{j} f(i . w)=f\left(\alpha_{j}^{-1} \cdot i \cdot w \cdot \alpha_{j}\right)=f\left(\alpha_{j}^{-1} \cdot i \cdot \alpha_{j} \cdot w \cdot w^{-1} \cdot \alpha_{j}^{-1} \cdot w \cdot \alpha_{j}\right) \\
=f\left(\alpha_{j}^{-1} \cdot i \cdot \alpha_{j} \cdot w\right) \kappa\left(w^{-1} \cdot \alpha_{j}^{-1} \cdot w \cdot \alpha_{j}\right)
\end{aligned}
$$

Or $\alpha_{j}^{-1} . i . \alpha_{j} . w \in \mathrm{GL}_{g}\left(\mathbb{Z}_{p}\right)$ et comme on a supposé $\kappa \in X(\mathrm{~T})^{+}, \kappa\left(w^{-1} \cdot \alpha_{j}^{-1} \cdot w \cdot \alpha_{j}\right) \in \mathbb{Z}_{p}$.

Le même argument montre la stabilité de $\mathcal{F}_{A}^{\mathrm{P}}[-\kappa]$.

REMARQUE 3.2. - Le réseau $R_{A}[\kappa]$ n'est en général pas stable sous les opérateurs $t^{\text {alg }}(\alpha)$. Ceci est à la source de quelques complications.

Si P est le parabolique standard de $\mathrm{GL}_{g}$ associé au $r$-uplet $\left(n_{1}, \ldots, n_{r}\right)$, on rappelle qu'on a posé $N_{j}=\sum_{i=1}^{j} n_{i}$. Soit $\alpha_{P}=\prod_{j=1}^{r-1} \alpha_{N_{j}}$. Définissons les projecteur $e_{\mathrm{P}}^{\text {alg }}=\lim _{n \rightarrow \infty} t^{\text {alg }}\left(\alpha_{\mathrm{P}}\right)^{n !}$ et $e_{\mathrm{P}}^{\text {top }}=\lim _{n \rightarrow \infty} t^{\text {top }}\left(\alpha_{\mathrm{P}}\right)^{n !}$.

Proposition 3.2. - Pour tout poids $\kappa \in X\left(\mathrm{~T}_{\mathrm{P}}\right)^{+}$, on a des isomorphismes de $\mathrm{T}_{\mathrm{P}}$-modules :

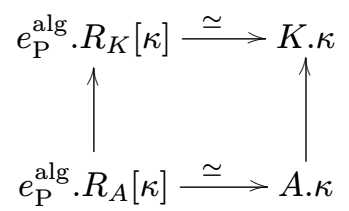

Démonstration. - La restriction à la grosse cellule P.P $\mathrm{P}^{0}$ est injective. Comme $\alpha_{\mathrm{P}}^{-1} \cdot \alpha_{\mathrm{P}}$ contracte $\mathrm{P}$ dans le parabolique opposé $\mathrm{P}^{0}$, toute fonction $f \in$ $e_{\mathrm{P}} \cdot R_{K}^{0}[\kappa]$ est nulle.

REMARQUE 3.3. - On a en fait $e_{\mathrm{P}}^{\mathrm{alg}} \cdot R_{A}[\kappa]=e_{\mathrm{P}}^{\mathrm{alg}} \cdot \tilde{R}_{A}[\kappa]$.

Proposition 3.3. - Pour tout poids $\kappa \in X\left(\mathrm{~T}_{\mathrm{P}}\right)^{++}$, on a des isomorphismes

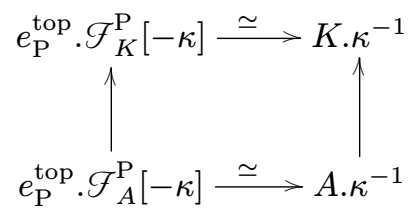

L'application $R_{A}[\kappa] \rightarrow \mathcal{F}_{A}^{\mathrm{P}}[-\kappa]$ induit donc un isomorphisme

$$
e_{\mathrm{P}}^{\mathrm{alg}} \cdot R_{A}[\kappa] \stackrel{\sim}{\rightarrow} e_{\mathrm{P}}^{\mathrm{top}} \cdot \mathcal{F}_{A}^{\mathrm{P}}[-\kappa] .
$$

TOME $140-2012-\mathrm{N}^{\mathrm{O}} 3$ 
Démonstration. - Soit $\mathrm{I}_{\mathrm{P}^{0}}$ le sous-groupe parahorique des matrices $M \in$ $\mathrm{GL}_{g}\left(\mathbb{Z}_{p}\right)$ dont la réduction modulo $p$ appartient à $\mathrm{P}^{0}\left(\mathbb{F}_{p}\right)$. On a la décomposition :

$$
\mathrm{GL}_{g}\left(\mathbb{Z}_{p}\right)=\coprod_{w \in W / W_{M}} \mathrm{I}_{\mathrm{P}^{\circ}} w S \mathrm{P}\left(\mathbb{Z}_{p}\right)
$$

On va montrer que pour tout $g \in \mathrm{GL}_{g}\left(\mathbb{Z}_{p}\right)$ et tout $f \in \mathcal{F}_{A}^{\mathrm{P}, 0}[-\kappa]$, il existe $N \in \mathbb{N}$ tel que $t\left(\alpha_{\mathrm{P}}\right)^{N} f(g) \in p A$. Ceci entraînera bien la nullité du module $e_{\mathrm{P}} \cdot \mathcal{F}_{A}^{\mathrm{P}, 0}[\kappa]$.

Soit $f \in \mathcal{F}_{A}^{\mathrm{P}, 0}[\kappa]$ et $g \in \mathrm{GL}_{g}\left(\mathbb{Z}_{p}\right)$. On a $g=i . w . p$ avec $i \in \mathrm{I}_{\mathrm{P}^{0}}, w \in W / W_{M}$ et $p \in \mathrm{P}\left(\mathbb{Z}_{p}\right)$.

Si $w \neq 1$, on a

$$
\begin{aligned}
t\left(\alpha_{\mathrm{P}}\right) f(i \cdot w) & =f\left(\alpha_{\mathrm{P}} \cdot i \cdot w \cdot \alpha_{\mathrm{P}}^{-1}\right) \\
& =f\left(\alpha_{\mathrm{P}} \cdot i \cdot \alpha_{\mathrm{P}}^{-1} \cdot w \cdot w^{-1} \cdot \alpha_{\mathrm{P}} \cdot w \cdot \alpha_{\mathrm{P}}^{-1}\right) \\
& =f\left(\alpha_{\mathrm{P}} \cdot i \cdot \alpha_{\mathrm{P}}^{-1} \cdot w\right) \kappa^{-1}\left(w^{-1} \cdot \alpha_{\mathrm{P}} \cdot w \cdot \alpha_{\mathrm{P}}^{-1}\right)
\end{aligned}
$$

On a bien $\alpha_{\mathrm{P}} \cdot i \cdot \alpha_{\mathrm{P}}^{-1} \cdot w \in \mathrm{GL}_{g}\left(\mathbb{Z}_{p}\right)$ et $\kappa^{-1}\left(w^{-1} \cdot \alpha_{\mathrm{P}} \cdot w \cdot \alpha_{\mathrm{P}}^{-1}\right) \in p \mathbb{Z}_{p}$ grâce à l'hypothèse $\kappa \in X\left(T_{\mathrm{P}}\right)^{++}$.

Reste le cas où $w=1$. Si $g \in \mathrm{P}^{0}\left(\mathbb{Z}_{p}\right) \mathrm{P}\left(\mathbb{Z}_{p}\right), e_{\mathrm{P}}^{\text {top }} f(g)=0$ car la conjugaison $\alpha_{\mathrm{P}} . \alpha_{\mathrm{P}}^{-1}$ contracte $\mathrm{P}^{0}\left(\mathbb{Z}_{p}\right) \mathrm{P}\left(\mathbb{Z}_{p}\right)$ dans $\mathrm{P}\left(\mathbb{Z}_{p}\right)$ et la fonction $f$ est nulle sur $\mathrm{P}\left(\mathbb{Z}_{p}\right)$.

Si $g \in \mathrm{I}_{\mathrm{P}^{0}} \backslash \mathrm{P}^{0}\left(\mathbb{Z}_{p}\right)$, il existe $n \in \mathbb{N}$ tel que $\alpha_{\mathrm{P}}^{n} g \alpha_{\mathrm{P}}^{-n} \in \mathrm{GL}_{g}\left(\mathbb{Z}_{p}\right) \backslash \mathrm{I}_{\mathrm{P}^{0}}\left(\mathbb{Z}_{p}\right)$ et on est ramené au cas où $w \neq 1$.

On peut également considérer l'action décalée par un élément $w \in W$,

$$
\begin{aligned}
t^{w}(\alpha): R_{K}[\kappa] & \rightarrow R_{K}[\kappa] \\
f & \mapsto f\left(\alpha^{-1} w . \alpha\right)
\end{aligned}
$$

Lemme 3.1. - Si le poids $\kappa$ est P-régulier et $w \notin W_{\mathrm{M}}$, pour toute fonction $f \in R_{A}[\kappa]$,

$$
t^{w}\left(\alpha_{\mathrm{P}}\right) \circ e_{\mathrm{P}} \cdot f \in p \tilde{R}_{A}[\kappa]
$$

Démonstration. - Comme la grosse cellule B.U $\mathrm{U}^{0}$ est dense et $\alpha_{\mathrm{P}}^{-1}$. $\alpha_{\mathrm{P}}$ contracte $\mathrm{B}$ dans le parabolique $\mathrm{P}^{0}$, il suffit de montrer que $t^{w}\left(\alpha_{\mathrm{P}}\right) f(g) \in$ $p A$ lorsque $g \in \mathrm{P}^{0}$. Or on a $t^{w}\left(\alpha_{\mathrm{P}}\right) f(g)=f\left(w \cdot w^{-1} \cdot \alpha_{\mathrm{P}}^{-1} \cdot w \cdot g \cdot \alpha_{\mathrm{P}}\right)=$ $\kappa\left(w^{-1} \cdot \alpha_{\mathrm{P}}^{-1} \cdot w \cdot \alpha_{\mathrm{P}}\right) f(w \cdot g)$. Comme $\kappa$ est régulier, $\kappa\left(w^{-1} \cdot \alpha_{\mathrm{P}}^{-1} \cdot w \cdot \alpha_{\mathrm{P}}\right) \in p \mathbb{Z}_{p}$. 


\section{Formes modulaires de Siegel}

\subsection{Généralités}

4.1.1. Formes modulaires arithmétiques. - Soit $N$ un entier positif , $\mathcal{N}$ un sous-groupe de $\mathrm{G}(\mathbb{Z} / N)$. Notons

$$
\Gamma_{\mathcal{N}}=\{g \in \mathrm{G}(\mathbb{Z}), g \bmod \mathbb{Z} / N \in \mathcal{N}\}
$$

le sous-groupe de congruence de niveau $\mathcal{N}$ de $\mathrm{G}(\mathbb{Z})$. Soit $A$ une $\mathbb{Z}[1 / N]$-algèbre. Considérons les foncteurs :

$$
\begin{aligned}
X: A-\mathrm{ALG} & \longrightarrow \mathrm{ENS} \\
R & \rightsquigarrow\left\{\left(\mathscr{G}, \lambda, \psi_{\mathcal{N}}\right) /{ }_{R}\right\} / \text { isom } \\
\mathcal{T}: A-\mathrm{ALG} & \longrightarrow \mathrm{ENS} \\
R & \rightsquigarrow\left\{\left(\mathscr{G}, \lambda, \psi_{\mathcal{N}}, \omega\right) / R\right\} / \text { isom }
\end{aligned}
$$

où

- $\mathscr{G} \rightarrow$ Spec $R$ est un schéma abélien de dimension $g$ sur Spec $R$ de section unité $e$.

$-\lambda$ est une polarisation principale.

- $\psi_{\mathcal{N}}$ est une structure de niveau $\mathcal{N}$. C'est une section de l'espace homogène sous $\mathrm{G}(\mathbb{Z} / N) / \mathcal{N}$,

$$
\operatorname{Isom}\left(\mathscr{G}[N],(\mathbb{Z} / N \mathbb{Z})^{2 g}\right) / \mathcal{N}
$$

où $\operatorname{Isom}\left(\mathscr{C}[N],(\mathbb{Z} / N \mathbb{Z})^{2 g}\right)$ est le torseur des isomorphismes symplectiques à un facteur de similitude près.

- $\omega: R^{g} \stackrel{\sim}{\rightarrow} e^{\star} \Omega_{\mathscr{Q} / R}^{1}$ est une trivialisation du faisceau conormal relatif à la section unité.

On dispose du morphisme d'« oubli de $\omega »: \Pi: \mathcal{T} \rightarrow X$.

Le groupe $\mathrm{GL}_{g} /{ }_{A}$ agit à gauche sur le foncteur $\mathcal{J}:$ si $g \in \mathrm{GL}_{g}(R)$ et $\left(\mathscr{G}, \lambda, \psi_{\mathcal{N}}, \omega\right) \in \mathcal{F}(R)$, on pose $g .\left(\mathscr{G}, \lambda, \psi_{\mathcal{N}}, \omega\right)=\left(\mathscr{G}, \lambda, \psi_{\mathcal{N}}, \omega \circ g^{-1}\right)$; cette action fait de $\mathcal{T}$ un $\mathrm{GL}_{g}$-torseur au dessus de $X$.

On définit les formes modulaires de Siegel arithmétiques comme suit.

DÉFINITION 4.1. - Une forme modulaire de Siegel F, de dimension $g$, niveau $\Gamma_{\mathcal{N}}$, poids $\kappa$, à coefficients dans $A$ est une loi qui à tout quintuplet $\left(R, \mathscr{G}, \lambda, \psi_{\mathcal{N}}, \omega\right)$, avec $R \in \mathrm{Ob}(A-$ alg $)$ et $\left(\mathscr{G}, \lambda, \psi_{\mathcal{N}}, \omega\right) \in \mathcal{T}(R)$, associe un élément de $R$ noté $F\left(R, \mathscr{G}, \lambda, \psi_{\mathcal{N}}, \omega\right)$ et qui vérifie les propriétés suivantes :

TOME $140-2012-\mathrm{N}^{\mathrm{O}} 3$ 
- Fonctorialité : Soit $R \stackrel{r}{\rightarrow} R^{\prime}$ un morphisme de A-algèbres. Pour tout diagramme cartésien

$$
\begin{array}{ccc}
\left(\mathscr{G}^{\prime}, \lambda^{\prime}, \psi_{\mathcal{N}}^{\prime}, \omega^{\prime}\right) & \rightarrow\left(\mathscr{G}, \lambda, \psi_{\mathcal{N}}, \omega\right) \\
\downarrow & \downarrow \\
\operatorname{Spec} R^{\prime} & \rightarrow & \operatorname{Spec} R
\end{array}
$$

on $\operatorname{ar}\left(F\left(\mathscr{G}, \lambda, \psi_{\mathcal{N}}, \omega\right)\right)=F\left(\mathscr{G}^{\prime}, \lambda^{\prime}, \psi_{\mathcal{N}}^{\prime}, \omega^{\prime}\right)$.

- Équation fonctionnelle :

$\forall t \in \mathrm{T}(R), \forall u \in \mathrm{U}^{0}(R), F\left(R, \mathscr{G}, \lambda, \psi_{\mathcal{N}}, \omega \circ t u\right)=\kappa^{\prime}(t) . F\left(R, \mathscr{G}, \lambda, \psi_{\mathcal{N}}, \omega\right)$.

On note $\mathrm{M}\left(\kappa, \Gamma_{\mathscr{N}}, A\right)$ le $A$-module des formes modulaires de Siegel de niveau $\Gamma_{\mathcal{N}}$, poids $\kappa$, à coefficients dans $A$.

On suppose dans toute la suite de ce travail que $\Gamma_{\mathcal{N}}$ est net.

C'est par exemple le cas si $\mathcal{N}=\{1\}$ et $N \geq 3$. Cette hypothèse sera utilisée de façon cruciale au numéro 6 . Les foncteurs $\mathcal{T}$ et $X$ sont alors représentables par des $A$-schémas encore notés $\mathcal{T}$ et $X$ ([3], chap. I).

Choisissons une compactification toroïdale $\bar{X}$ du schéma $X$ ([3], IV. 6, th. 6.7). Le schéma abélien universel $\mathscr{G} \rightarrow X$ s'étend en un schéma semi-abélien $\mathscr{G} \rightarrow \bar{X}$. Soit $e: \bar{X} \rightarrow \mathscr{C}$ sa section unité.

On prolonge alors $\mathcal{T}$ en un $\mathrm{GL}_{g}$-torseur au-dessus de $\bar{X}$ en posant :

$$
\overline{\mathcal{J}}=\operatorname{Isom}\left(\theta_{\bar{X}}^{g}, e^{\star} \Omega_{\mathscr{Q} / \bar{X}}^{1}\right) \text {. }
$$

Le groupe $\mathrm{GL}_{g}$ agit sur $\Theta_{\overline{\mathcal{J}}}$ a gauche par $g . f(\mathscr{A}, \omega)=f(\mathscr{G}, \omega \circ g)$. Le faisceau $\Pi_{\star} \theta_{\overline{\mathcal{J}} / \mathrm{U}^{0}}$ sur $\bar{X}$ est muni d'une action de T. Pour tout $\kappa \in X(\mathrm{~T})$, on définit le faisceau $\omega^{\kappa}=\Pi_{\star} \theta_{\overline{\mathcal{T}} / \mathrm{U}^{0}}\left[\kappa^{\prime}\right]$ en prenant les $\kappa^{\prime}$-variants. On trouve alors que $\mathrm{M}\left(\kappa, \Gamma_{\mathcal{N}}, A\right)=\mathrm{H}^{0}\left(X, \omega^{\kappa}\right)$.

Le principe de Koecher ([3], V.1, prop. 1.5 ) entraîne :

$$
\mathrm{H}^{0}\left(\bar{X}, \omega^{\kappa}\right)=\mathrm{H}^{0}\left(X, \omega^{\kappa}\right) \text {. }
$$

On dit que $F \in \mathrm{M}\left(\kappa, \Gamma_{\mathscr{N}}, A\right)$ est cuspidale si elle s'annule le long de $\bar{X} \backslash X$. Cette définition ne dépend pas du choix de $\bar{X}$. On note $\mathrm{S}\left(\kappa, \Gamma_{\mathscr{N}}, A\right) \subset \mathrm{M}\left(\kappa, \Gamma_{\mathscr{N}}, A\right)$ le sous- $A$-module des formes cuspidales.

REMARQUE 4.1. - Si $g=2$ et $\kappa=\left(k_{1}, k_{2}\right)$ est dominant $\left(k_{1} \geq k_{2}\right)$, considérons la représentation sym ${ }^{k_{1}-k_{2}} \mathrm{St} \otimes_{\mathbb{Z}} \operatorname{det}^{k_{2}} \mathrm{du}$ groupe $\mathrm{GL}_{2} / \mathbb{Z}$, où sym est vu comme quotient du produit tensoriel. C'est une représentation de plus haut poids $\kappa$. Soit par ailleurs la représentation $R_{\mathbb{Z}}\left[\kappa^{\prime}\right]$ défini au numéro 3.1.1. C'est aussi une représentation de plus haut poids $\kappa$. Par réciprocité de Frobenius, on a une application

$$
\operatorname{sym}^{k_{1}-k_{2}} \mathrm{St} \otimes \operatorname{det}^{k_{2}} \rightarrow R_{\mathbb{Z}}\left[\kappa^{\prime}\right]
$$


et on vérifie facilement que cette application est un isomorphisme. Un tel isomorphisme induit alors un isomorphisme

$$
\omega^{\kappa} \simeq \operatorname{sym}^{k_{1}-k_{2}} e^{\star} \Omega_{\mathscr{G} / \bar{X}}^{1} \otimes \operatorname{det}^{k_{2}} e^{\star} \Omega_{\mathscr{G} / \bar{X}}^{1} .
$$

En effet, au dessus de $\overline{\mathcal{T}}$ on dispose d'une trivialisation $\theta_{\overline{\mathcal{T}}}^{2} \stackrel{\omega}{\simeq} e^{\star} \Omega_{\mathscr{C} / \bar{X}}^{1}$. Soit $U$ un ouvert de $\bar{X}$. Une section de $\operatorname{sym}^{k_{1}-k_{2}} e^{\star} \Omega_{\mathscr{C} / \bar{X}}^{1} \otimes \operatorname{det}^{k_{2}} e^{\star} \Omega_{\mathscr{C} / \bar{X}}^{1}$ sur $U \times_{\bar{X}} \overline{\mathcal{J}}$ est une loi fonctorielle $F$ qui à tout point $(\mathscr{C} / R, \omega)$ à valeur dans $U \times_{\bar{X}} \overline{\mathcal{T}}$ associe un vecteur dans la représentation de $\mathrm{GL}_{2} / R: \mathrm{sym}^{k_{1}-k_{2}} \mathrm{St} \otimes \operatorname{det}^{k_{2}}$ et qui satisfait l'équation fonctionnelle (condition de descente à $U): F\left(A, \omega \circ g^{-1}\right)=g \cdot F(A, \omega)$.

On dispose par ailleurs d'un isomorphisme sym ${ }^{k_{1}-k_{2}} \mathrm{St} \otimes \operatorname{det}^{k_{2}} \simeq R_{\mathbb{Z}}\left[\kappa^{\prime}\right]$ et on peut alors voir $F$ comme une loi fonctorielle qui à $(\mathscr{G} / R, \omega)$ associe une fonction sur $\mathrm{GL}_{2}$ telle que $\forall g \in \mathrm{GL}_{2}, \forall b \in \mathrm{B}^{0}, \quad F(\mathscr{G} / R, \omega)(g b)=\kappa^{\prime}(b) F(\mathscr{G} / R, \omega)(g)$ et qui vérifie de plus l'équation fonctionnelle $F\left(A, \omega \circ g^{-1}\right)=g \cdot F(A, \omega)$. On vérifie alors que $\ell_{c a n}(F)$ est une section du faisceau $\omega^{\kappa}$ et que l'application $F \mapsto \ell_{c a n}(F)$ est l'isomorphisme recherché.

4.1.2. Formes modulaires avec Nebentypus. — Soit $q$ un nombre premier, $q \nmid$ $N$. Soit $A$ une $\mathbb{Z}\left[\frac{1}{N q}\right]$-algèbre. Définissons des foncteurs :

$$
\begin{aligned}
X_{0, q}: A-\mathrm{ALG} & \longrightarrow \mathrm{ENS} \\
R & \rightsquigarrow\left(\mathscr{G}, \lambda, \psi_{\mathcal{N}}, H\right) / \text { isom } \\
X_{1, q}: A-\mathrm{ALG} & \longrightarrow \mathrm{ENS} \\
R & \rightsquigarrow\left(\mathscr{G}, \lambda, \psi_{\mathcal{N}}, Q\right) / \text { isom }
\end{aligned}
$$

où $\mathscr{G}$ est un schéma abélien de dimension $g$ sur $R, \lambda$ est un polarisation principale, $\psi_{\mathcal{N}}$ une structure de niveau $\Gamma_{\mathcal{N}}, H$ est un sous groupe de rang $q$ de $\mathscr{G}$ et $Q$ un $R$-point de $\mathscr{C}[q]$ d'ordre $q$.

On note $\Gamma^{0}(q) \subset \Gamma^{1}(q) \subset \mathrm{G}(\mathbb{Z})$ les sous-groupes de congruences parahorique de Klingen et parahorique de Klingen strict :

$$
\begin{aligned}
& \Gamma^{0}(q)=\left\{M \in \mathrm{G}(\mathbb{Z}), M \bmod p \in \mathrm{K}\left(\mathbb{F}_{p}\right)\right\} \\
\text { et } \quad \Gamma^{1}(q) & =\left\{M \in \mathrm{G}(\mathbb{Z}), M \bmod p \in \mathrm{K}^{+}\left(\mathbb{F}_{p}\right)\right\}
\end{aligned}
$$

On note alors $\Gamma_{\mathcal{N}}^{0}(q)=\Gamma_{\mathcal{N}} \cap \Gamma^{0}(q)$ et $\Gamma_{\mathcal{N}}^{1}(q)=\Gamma_{\mathcal{N}} \cap \Gamma^{1}(q)$. Les foncteurs $X_{0, q}$ et $X_{1, q}$ sont donc associés aux groupes de congruences $\Gamma_{\mathcal{N}}^{0}(q)$ et $\Gamma_{\mathcal{N}}^{1}(q)$.

Le groupe $(\mathbb{Z} / q \mathbb{Z})^{\times}$agit sur $X_{1, q}$ et $X_{1, q} \rightarrow X_{0, q}$ est un revêtement de groupe $(\mathbb{Z} / q \mathbb{Z})^{\times}$.

Les foncteurs $X_{1, q}$ et $X_{0, q}$ sont représentables par des schémas encore notés de la même manière.

Soit $\bar{X}_{0, q}$ une compactification toroïdale de $X_{0, q}$. D'après les resultats de [21], chap. 5, le sous-groupe universel $H \hookrightarrow \mathscr{C}[q]$ au dessus de $X_{0, q}$ se prolonge 
en un groupe fini et plat au dessus de $\bar{X}_{0, q}$. On définit alors la compactification toroïdale de $X_{1, q}$ en posant

$$
\bar{X}_{1, q}=\operatorname{Isom}_{\bar{X}_{0, q}}(\mathbb{Z} / q \mathbb{Z}, H) .
$$

Le morphisme $f: \bar{X}_{1, q} \rightarrow \bar{X}_{0, q}$ est un revêtement étale de groupe $(\mathbb{Z} / q \mathbb{Z})^{\times}$.

Pour tout poids $\kappa \in X(\mathrm{~T})$, on dispose de faisceaux cohérents (notés indifféremment de la même manière si cela ne prête à confusion) $\omega^{\kappa}$ sur $\bar{X}_{1, q}$ et $\bar{X}_{0, q}$ d'après la construction du numéro 4.1.1.

Pour tout caractère $\chi:(\mathbb{Z} / q \mathbb{Z})^{\times} \rightarrow A^{\times}$soit $\theta_{\bar{X}_{0, q}}(\chi)$ le sous-faisceau de $f_{\star} \vartheta_{\bar{X}_{1, q}}$ des section $\chi$-variantes. C'est un faisceau inversible de $\theta_{\bar{X}_{0, q}}$-module. Pour tout faisceau cohérent $\mathcal{F}$ de $\vartheta_{\bar{X}_{0, q}}$-module on note

$$
\mathcal{F}(\chi)=\mathcal{F} \otimes \vartheta_{\bar{X}_{0, q}} \vartheta_{\bar{X}_{0, q}}(\chi)
$$

DÉfinition 4.2. - Le A-module $\mathrm{H}^{0}\left(\bar{X}_{0, q}, \omega^{\kappa}(\chi)\right)$ est le module des formes modulaires de Siegel de genre $g$, poids $\kappa$, niveau $\Gamma_{\mathcal{N}}^{0}(q)$ et caractère $\chi$. Il est noté $\mathrm{M}\left(\kappa, \Gamma_{\mathcal{N}}^{0}(q), \chi, A\right)$.

Le sous-module $\mathrm{H}_{\text {cusp }}^{0}\left(\bar{X}_{0, q}, \omega^{\kappa}(\chi)\right)$ des formes cuspidales est noté $\mathrm{S}\left(\kappa, \Gamma_{\mathcal{N}}^{0}(q), \chi, A\right)$

4.1.3. Formes modulaires $p$-adiques. - Soit $p$ un nombre premier ne divisant pas $N$. Nous reprenons les notations de la section précédente en posant $A=\mathbb{Z}_{p}$. Le schéma $\bar{X}$ est donc une compactification torö̈dale de l'espace des modules sur $\mathbb{Z}_{p}$ des variétes abéliennes principalement polarisées avec structure de niveau $\mathcal{N}$.

Pour tout schéma $Y$ sur $\mathbb{Z}_{p}$ on note $Y_{m}$ le produit fibré $Y \times_{\mathbb{Z}_{p}} \mathbb{Z} / p^{m} \mathbb{Z}$.

On a aussi construit des faisceaux modulaires $\omega^{\kappa}$ sur $\bar{X}$ tels que $\mathrm{M}\left(\kappa, N, \mathbb{Z}_{p}\right)=\mathrm{H}^{0}\left(\bar{X}, \omega^{\kappa}\right)$.

Remarque 4.2. - Sur $\bar{X}_{m}$, pour tout poids $\kappa \in X(\mathrm{~T})$, on dispose de deux faisceaux modulaires : le premier obtenu à l'aide du torseur $\overline{\mathcal{J}}_{m}$ (voir le numéro 4.1.1), le second obtenu par changement de base depuis $\bar{X}$. Il résulte du théorème d'annulation de Kempf ([9], II. 4.6) que $R_{\mathbb{Z}_{p}}\left[\kappa^{\prime}\right] \otimes \mathbb{Z} / p^{m} \mathbb{Z}=R_{\mathbb{Z} / p^{m} \mathbb{Z}}\left[\kappa^{\prime}\right]$ et les deux faisceaux envisagés coïncident donc.

L'invariant de Hasse $H$ est un élément de $\mathrm{M}((p-1, p-1, \ldots, p-1), N, \mathbb{Z} / p \mathbb{Z})$. On sait qu'une certaine puissance $H^{t}$ de $H$ se relève en un élément $E \in \mathrm{M}\left(t(p-1, \ldots, p-1), N, \mathbb{Z}_{p}\right)$. Nous notons $\& \hookrightarrow \bar{X}$ le lieu où $E$ est inversible. Le schéma $\varnothing$ dépend du choix de $E$, par contre, pour tout $m \geq 1$ le schéma $\oint_{m}$ ne dépend pas du relèvement de $H$. C'est le lieu ordinaire de $\bar{X}_{m}$. Nous appellerons $\phi_{\infty}$ la complétion formelle de $\phi$ le long de $\phi_{1}$.

Pour tout entier $n \geq 0$, nous définissons le schéma :

$$
\mathcal{J}_{m, n}=\operatorname{Isom}_{\phi_{m}}\left(\mu_{p^{n}}^{g}, \mathscr{G}\left[p^{n}\right]^{0}\right) \text {. }
$$


Le groupe $\mathrm{GL}_{g}\left(\mathbb{Z} / p^{n}\right)=\operatorname{Aut}\left(\mu_{p^{n}}^{g}\right)$ agit sur $\mathcal{T}_{m, n}$ à droite de la façon suivante :

$$
g .\left(\mathscr{G}, \psi_{n}: \mu_{p^{n}}^{g} \simeq \mathscr{G}\left[p^{n}\right]^{0}\right)=\left(\mathscr{G}, \psi_{n} \circ g\right) .
$$

Le morphisme naturel $\mathcal{J}_{m, n} \rightarrow \phi_{m}$ fait de $\mathcal{J}_{m, n}$ un revêtement étale de groupe $\mathrm{GL}_{g}\left(\mathbb{Z} / p^{n}\right)$. On pose $\mathcal{J}_{m, \infty}=\lim _{n \geq 0} \mathcal{J}_{m, n}$. C'est un pro-revêtement étale de $\phi_{m}$ de groupe $\mathrm{GL}_{g}\left(\mathbb{Z}_{p}\right)$. On note finalement $\mathcal{J}_{\infty}=\operatorname{colim}_{m \geq 0} \mathcal{J}_{m, \infty}$. C'est un pro-revêtement étale de $\phi_{\infty}$ de groupe $\mathrm{GL}_{g}\left(\mathbb{Z}_{p}\right)$, appelé tour d'Igusa.

On appelle pointe de $\mathcal{T}_{m, n}$ le sous-schéma fermé image inverse de $\bar{X} \backslash X$.

Les différents schémas introduits s'organisent de la manière suivante :

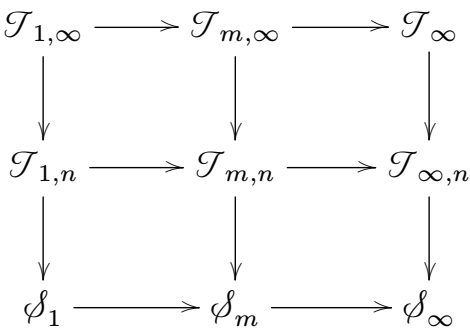

Posons $V_{m, n}=H^{0}\left(\mathcal{J}_{m, n}, \mathcal{\vartheta}_{\mathcal{J}_{m, n}}\right), V_{m}=H^{0}\left(\mathcal{J}_{m, \infty}, \mathscr{\vartheta}_{\mathcal{J}_{m, \infty}}\right)$ et $V_{\infty}=$ $\lim _{m \geq 1} V_{m}$.

Le groupe $\mathrm{GL}_{g}\left(\mathbb{Z}_{p}\right)$ agit à gauche sur $V_{m, n}$ à travers son quotient $\mathrm{GL}_{g}\left(\mathbb{Z} / p^{n}\right)$ par la formule $g \cdot f\left(\mathscr{G} / R, \psi_{n}\right)=f\left(\mathscr{G} / R, \psi_{n} \circ g\right)$.

Cette action induit des actions à gauche de $\mathrm{GL}_{g}\left(\mathbb{Z}_{p}\right)$ sur $V_{m}$ et sur $V_{\infty}$.

Soit $\mathrm{P}$ un parabolique standard de $\mathrm{GL}_{g}$ et $\mathrm{Q}=\omega_{0} \cdot{ }^{t} \mathrm{P} \cdot \omega_{0}$. On définit à présent l'algèbre des formes de Siegel $p$-adiques pour $\mathrm{P}$ et son idéal des formes cuspidales.

DÉfInition 4.3 (Formes $p$-adiques). — $\quad$ 1. L'ensemble $V_{\infty}^{S P}$ des fonctions régulières invariantes sous $S \mathrm{P}\left(\mathbb{Z}_{p}\right)$ est l'anneau des formes modulaires de Siegel $p$-adique pour le parabolique $\mathrm{P}$, de niveau $\mathcal{N}$.

2. Pour tout caractère $\kappa$ du tore $\mathrm{T}_{\mathrm{Q}}\left(\mathbb{Z}_{p}\right)$, on appelle forme modulaire de Siegel p-adique pour le parabolique $\mathrm{P}$, de niveau $\mathcal{N}$ et de poids $\kappa$, un élément du module $V_{\infty}^{S \mathrm{P}}\left[-\kappa^{\prime}\right]$.

3. Si $\mathrm{P}=\mathrm{B}$, on dit simplement que $V_{\infty}^{\mathrm{U}}$ est l'algèbre des formes de Siegel $p$-adiques de niveau $\mathcal{N}$ et que $V_{\infty}^{\mathrm{U}}\left[-\kappa^{\prime}\right]$ est le module des formes de Siegel p-adiques de niveau $\mathcal{N}$ et poids $\kappa$.

4. La partie cuspidale $V_{\mathrm{cusp}, \infty}^{S \mathrm{P}}$ est l'idéal de $V_{\infty}^{S \mathrm{P}}$ des formes qui s'annulent aux pointes. 
4.1.4. Formes modulaires p-adiques avec nebentypus. - Soit $q$ un nombre premier ne divisant pas $N p$. Soit $A$ l'anneau des entiers d'une extension finie de $\mathbb{Q}_{p}$, d'uniformisante $\pi$. Considérons les schémas $\bar{X}_{1, q} \rightarrow \bar{X}_{0, q} \rightarrow \bar{X}_{A} \rightarrow \operatorname{Spec} A$. Par extension des scalaires à $A$ et produit fibré avec $\bar{X}_{1, q}$ ou $\bar{X}_{0, q}$ au dessus de $\bar{X}_{A}$, on obtient les schémas suivants :

$-\phi_{1, q} \rightarrow \phi_{0, q} \rightarrow \phi_{A}$, ce sont les ouverts de non annulation de $E$.

- Pour tout $m \in \mathbb{N} \cup\{\infty\},\left(\phi_{1, q}\right)_{m} \rightarrow\left(\phi_{0, q}\right)_{m} \rightarrow \phi_{m} \rightarrow$ Spec $A / \pi^{m}$, les lieux ordinaires de $\left(\bar{X}_{1, q}\right)_{A / \pi^{m}},\left(\bar{X}_{0, q}\right)_{A / \pi^{m} A}$ et $\bar{X}_{A / \pi^{m}}$ qui sont aussi les réductions modulo $\pi^{m}$ de $\phi_{1, q}, \phi_{0, q}$ et $\phi_{A}$.

- Pour tous $m, n \in \mathbb{N} \cup\{\infty\}$, les tours d'Igusa $\left(\mathcal{J}_{1, q}\right)_{m, n} \rightarrow\left(\mathcal{J}_{0, q}\right)_{m, n} \rightarrow$ $\left(\mathcal{J}_{m, n}\right)_{A}$.

Pour $m \in \mathbb{N} \cup \infty$, on note $V_{1, q, m}=\mathrm{H}^{0}\left(\left(\mathcal{J}_{1, q}\right)_{\infty, m}, \mathcal{\vartheta}_{\left(\mathcal{J}_{1, q}\right)_{\infty, m}}\right)$. Le groupe $(\mathbb{Z} / q \mathbb{Z})^{\times}$agit sur le module $V_{1, q, m}$. Cette action est parfois appelée action des opérateurs diamant.

DÉFInition 4.4. - Soit $\mathrm{P}$ un parabolique standard de $\mathrm{GL}_{g}, \mathrm{Q}=\omega_{0} \cdot{ }^{t} \mathrm{P} \cdot \omega_{0}$ et $\chi:(\mathbb{Z} / q \mathbb{Z})^{\times} \rightarrow A^{\times}$un caractère.

1. On appelle $V_{1, q, \infty}^{S \mathrm{P}}[\chi]$ le $A$-module des formes modulaires $p$-adique pour le parabolique $\mathrm{P}$, de niveau $\Gamma_{\mathcal{N}}^{0}(q)$ et caractère $\chi$.

2. Pour tout poids $\kappa \in X\left(\mathrm{~T}_{\mathrm{Q}}\right)$, on appelle $V_{1, q, \infty}^{S \mathrm{P}}\left[\chi,-\kappa^{\prime}\right]$ le A-module des formes modulaires $p$-adique pour le parabolique $\mathrm{P}$, de poids $\kappa$, de niveau $\Gamma_{\mathcal{N}}^{0}(q)$ et caractère $\chi$.

3. On note $V_{1, q, \infty, \mathrm{cusp}}^{S \mathrm{P}}[\chi]$ le sous-module de $V_{1, q, \infty}^{S \mathrm{P}}[\chi]$ des formes cuspidales.

4.2. Comparaison des formes classiques et des formes $p$-adiques. - Pour la simplicité de l'exposition, nous nous restreignons dans cette sous-partie aux formes sans nebentypus. Toute la discussion se généralise immédiatement aux formes avec un nebentypus.

4.2.1. Construction des morphismes. - Soit $B$ une $\mathbb{Z} / p^{m}$-algèbre, soit $\mathscr{G} \stackrel{\pi}{\rightarrow}$ Spec $B$ un schéma semi-abélien ordinaire de dimension $g$, de section unité $e$ et soit $\psi_{n}: \mu_{p^{n}}^{g} \stackrel{\sim}{\rightarrow}\left[p^{n}\right]^{0}$ une rigidification. Si $n \geq m, \psi_{n}$ induit un isomorphisme au niveau des algèbres de Lie :

$$
\mathscr{L}\left(\psi_{n}\right): \mathscr{L} i e\left(\mu_{p^{n}}^{g}\right) \rightarrow \mathscr{L} i e(\mathscr{G}) .
$$

Soit $d_{c a n}: B^{g} \rightarrow e^{\star} \Omega_{\mu_{p^{n}}^{g} / B}^{1}$ l'application qui envoie la base canonique de $B^{g}$ sur $\left(d t_{1} / t_{1}, \ldots, d t_{g} / t_{g}\right)$. On définit alors un isomorphisme :

$$
\omega\left(\psi_{n}\right): B^{g} \stackrel{d_{\text {can } n}}{\longrightarrow} e^{\star} \Omega_{\mu_{p^{n}}^{g} / B}^{1} \stackrel{{ }^{t}}{\mathcal{L}\left(\psi_{n}\right)^{-1}} e^{\star} \Omega_{\mathscr{Q} / B}^{1} .
$$

Si $g \in \mathrm{GL}_{g}\left(\mathbb{Z} / p^{n}\right)$, on a par définition $g . \psi_{n}=\psi_{n} \circ g$ et donc $\omega\left(g . \psi_{n}\right)=$ $\omega\left(\psi_{n}\right) \circ^{t} g^{-1}$. 
On dispose donc de diagrammes naturels pour $n \geq m$ :

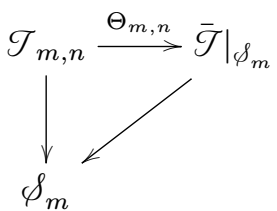

On peut définir une nouvelle action, à gauche, de $\mathrm{GL}_{g}\left(\mathbb{Z} / p^{n}\right)$ sur $\mathcal{J}_{m, n}$ en posant :

$$
g \star\left(\mathscr{G}, \psi_{n}: \mu_{p^{n}}^{g} \simeq \mathscr{G}\left[p^{n}\right]^{0}\right)=\left(\mathscr{C}, \psi_{n} \circ^{t} g\right) .
$$

Le schéma en groupes $\mathrm{GL}_{g} / \mathbb{Z} / p^{m}$ agit à gauche sur $\left.\overline{\mathcal{T}}\right|_{\phi_{m}}$. Le morphisme $\Theta_{m, n}$ est compatible avec ses actions à gauche et le morphisme naturel de schémas en groupes $\mathrm{GL}_{g}\left(\mathbb{Z} / p^{n}\right) \rightarrow \mathrm{GL}_{g} / \mathbb{Z} / p^{m}$.

En passant à la limite le diagramme précédent, on obtient :
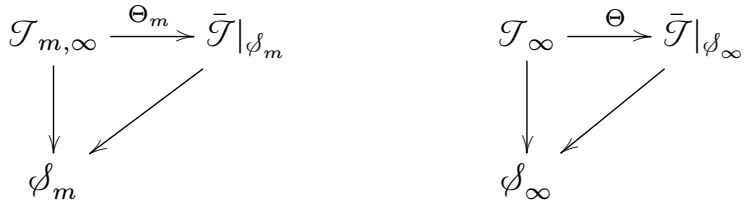

Par définition $\mathrm{H}^{0}\left(\phi_{m}, \omega^{\kappa}\right)=\mathrm{H}^{0}\left(\varnothing, \omega^{\kappa} \otimes_{\mathbb{Z}_{p}} \mathbb{Z} / p^{m} \mathbb{Z}\right)$ et $\mathrm{H}^{0}\left(\phi_{\infty}, \omega^{\kappa}\right)=$ $\lim _{m \geq 1} \mathrm{H}^{0}\left(\phi_{m}, \omega^{\kappa}\right)$.

Pour tout parabolique standard $\mathrm{P}$ et $\mathrm{Q}=\omega_{0} \cdot{ }^{t} \mathrm{P} \cdot \omega_{0}$ et tout poids $\kappa \in X\left(\mathrm{~T}_{\mathrm{Q}}\right)$, les applications $\Theta_{m}$ induisent des morphismes $\Theta_{m, \mathrm{P}}^{\star}: \mathrm{H}^{0}\left(\phi_{m}, \omega^{\kappa}\right) \rightarrow V_{m}^{S \mathrm{P}}\left[-\kappa^{\prime}\right]$.

Ces applications sont des isomorphismes lorsque $\mathrm{P}=\mathrm{GL}_{g}$. En général, elles ne sont ni injectives, ni surjectives.

À la limite on dispose d'une application injective $\Theta_{\mathrm{P}}^{\star}: \mathrm{H}^{0}\left(\phi_{\infty}, \omega^{\kappa}\right) \rightarrow$ $V_{\infty}^{S \mathrm{P}}\left[-\kappa^{\prime}\right]$.

Localement pour la topologie étale sur $\phi_{\infty}$, cette application est isomorphe à l'application $i: R_{\phi_{\infty}}\left[\kappa^{\prime}\right] \rightarrow \mathcal{F}_{\&_{\infty}}^{\mathrm{P}}\left[-\kappa^{\prime}\right]$ du numéro 3.1.2.

La situation se résume en écrivant les chaînes de morphismes :

$$
\begin{gathered}
\mathrm{M}\left(\kappa, N, \mathbb{Z} / p^{m} \mathbb{Z}\right) \hookrightarrow \mathrm{H}^{0}\left(\phi_{m}, \omega^{\kappa}\right) \stackrel{\Theta_{m, \mathrm{P}}^{\star}}{\rightarrow} V_{m}^{S \mathrm{P}}\left[-\kappa^{\prime}\right] \\
\mathrm{M}\left(\kappa, N, \mathbb{Z}_{p}\right) \hookrightarrow \mathrm{H}^{0}\left(\phi, \omega^{\kappa}\right) \hookrightarrow \mathrm{H}^{0}\left(\phi_{\infty}, \omega^{\kappa}\right) \stackrel{\Theta_{\mathrm{P}}^{\star}}{\rightarrow} V_{\infty}^{S \mathrm{P}}\left[-\kappa^{\prime}\right]
\end{gathered}
$$

Remarque 4.3. - On a bien sûr :

$$
\mathrm{H}^{0}\left(\phi, \omega^{\kappa}\right)=\bigcup_{r \geq 0} \frac{\mathrm{M}\left(\kappa+t r .(p-1, \ldots, p-1), N, \mathbb{Z}_{p}\right)}{E^{r}} .
$$


4.2.2. L'isomorphisme fondamental. - On rappelle que $\mathrm{P}$ est un parabolique standard de $\mathrm{GL}_{g}$ et que $\mathrm{P}^{0}$ désigne son parabolique opposé. Notons $\mathcal{J}_{m, n} / \mathrm{P}$ pour $\mathcal{J}_{m, n} / \mathrm{P}\left(\mathbb{Z} / p^{n}\right)$ (pour l'action à droite) et $\mathcal{J}_{m, n} / S \mathrm{P}$ pour $\mathcal{J}_{m, n} / S \mathrm{P}\left(\mathbb{Z} / p^{n}\right)$.

Si $n \geq m$, nous pouvons construire un $\mathrm{T}_{\mathrm{P}}$-torseur $\Omega_{\mathrm{P}}$ et des fibrés en droite $\Omega_{\mathrm{P}}^{\kappa}$ au dessus de $\mathcal{J}_{m, n} / \mathrm{P}$. Considérons en effet le diagramme commutatif

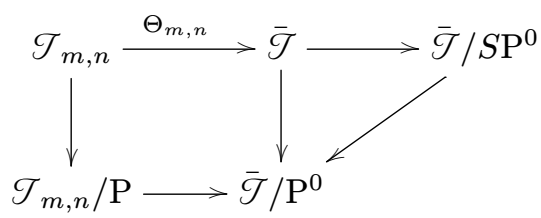

Posons $\Omega_{\mathrm{P}}=\mathcal{J}_{m, n} / \mathrm{P} \times \overline{\mathcal{T}} / \mathrm{P}^{0} \overline{\mathcal{T}} / S \mathrm{P}^{0}$ et notons $g$ la première projection. Les $R$-points de $\Omega_{\mathrm{P}} \times_{\bar{X}} X$ sur l'ouvert modulaire complémentaire des pointes sont donc simplement les classes d'isomorphismes de

- Une variété abélienne ordinaire principalement polarisée $\mathscr{G} \rightarrow \operatorname{Spec} R$.

- Un P-drapeau $\psi_{n}: \mu_{p^{n}}^{g} \simeq \mathscr{C}\left[p^{n}\right]^{0} \bmod \mathrm{P}$.

- Un $\mathrm{P}^{0}$-drapeau sur $e^{\star} \Omega_{G / R}^{1}$ avec une base du déterminant des gradués (c'est localement la $S \mathrm{P}^{0}$-orbite d'un isomorphisme $\omega: R^{g} \simeq e^{\star} \Omega_{\mathscr{G} / R}^{1}$ ) avec la condition de compatibilité $\omega\left(\psi_{n}\right)=\omega \bmod \mathrm{P}^{0}$.

Le tore $\mathrm{T}_{\mathrm{P}}=\mathrm{P}^{0} / S \mathrm{P}^{0}$ agit sur le choix de la base du déterminant des gradués.

On dispose à présent d'un diagramme commutatif

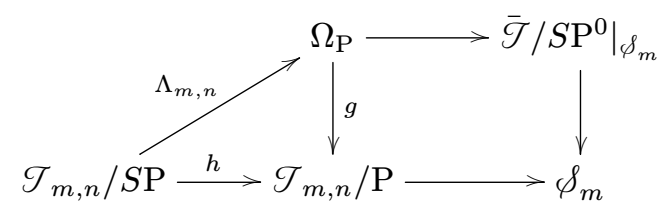

Le morphisme $\Lambda_{m, n}$ est induit par $\Theta_{m, n}$, et $g$ est la projection naturelle. Pour tout $\kappa \in X\left(\mathrm{~T}_{\mathrm{Q}}\right)$ on définit le fibré en droite :

$$
\Omega_{\mathrm{P}}^{\kappa}=g_{\star} \theta_{\Omega_{\mathrm{P}}}\left[\kappa^{\prime}\right]
$$

Proposition 4.1. - L'application $\Lambda_{m, n}$ induit un isomorphisme :

$$
\mathrm{H}^{0}\left(\mathcal{T}_{m, n} / \mathrm{P}, \Omega_{\mathrm{P}}^{\kappa}\right) \stackrel{\sim}{\rightarrow} V_{m, n}^{S \mathrm{P}}\left[-\kappa^{\prime}\right]
$$

Démonstration. - D'une part $\mathcal{J}_{m, n} / S \mathrm{P}$ est un revêtement étale de $\mathcal{T}_{m, n} / \mathrm{P}$ de groupe $\mathrm{T}_{\mathrm{P}}\left(\mathbb{Z} / p^{n}\right)$, d'autre part $\Omega_{\mathrm{P}}$ est un $\mathrm{T}_{\mathrm{P}}$-torseur. Localement pour la topologie étale sur $\mathcal{J}_{m, n} / \mathrm{P}$, le morphisme $\Lambda_{m, n}$ est donné par le morphisme naturel, tordu par l'automorphisme exterieur ${ }^{t}()^{-1}$, de schémas en groupes $\mathrm{T}_{\mathrm{P}}\left(\mathbb{Z} / p^{n}\right) \stackrel{t}{\stackrel{(}{()^{-1}}} \mathrm{~T}_{\mathrm{P}}$ qui induit un isomorphisme entre les fonctions polynomiales 
homogènes de poids $\kappa^{\prime}$ sur $\mathrm{T}_{\mathrm{P}}$ et les fonctions homogènes de poids $-\kappa^{\prime}$ sur $\mathrm{T}_{\mathrm{P}}\left(\mathbb{Z} / p^{n}\right)$ pour tout $\kappa \in X\left(\mathrm{~T}_{\mathrm{Q}}\right)$.

REMARQUe 4.4. - Cet isomorphisme établit le lien crucial entre la tour d'Igusa (et les formes p-adiques) et un objet différentiel (et les formes arithmétiques).

L'image inverse du faisceau $\omega^{\kappa}$ par le morphisme $\mathcal{J}_{m, n} / \mathrm{P} \rightarrow \phi_{m}$ définit un faisceau que nous notons encore $\omega^{\kappa}$ si le contexte est clair. Tâchons d'éclairer la relation entre les faisceaux $\omega^{\kappa}$ et $\Omega_{\mathrm{P}}^{\kappa}$ sur $\mathcal{J}_{m, n} / \mathrm{P}$. On a une immersion fermée $\mathrm{T}_{\mathrm{P}}$-équivariante de $\mathcal{J}_{m, n} / \mathrm{P}$-schémas :

$$
\Omega_{\mathrm{P}} \hookrightarrow \overline{\mathcal{T}} / S \mathrm{P}^{0} \times_{\phi_{m}} \mathcal{T}_{m, n} / \mathrm{P}
$$

qui est, localement pour la topologie de Zariski sur $\mathcal{J}_{m, n} / \mathrm{P}$, isomorphe à l'inclusion $\mathrm{P}^{0} / S \mathrm{P}^{0} \hookrightarrow \mathrm{GL}_{g} / S \mathrm{P}^{0}$. Pour tout $\kappa \in X\left(\mathrm{~T}_{\mathrm{Q}}\right)$, on en déduit un morphisme

$$
\text { res }: \omega^{\kappa} \rightarrow \Omega_{\mathrm{P}}^{\kappa} \text {. }
$$

Cette application est un analogue relatif de l'application res du numéro 3.1.1. Pour $\kappa$ dominant, res est surjective. On note le noyau $\omega_{0}^{\kappa}$. On a donc une suite exacte de faisceaux cohérents sur $\mathcal{J}_{m, n} / \mathrm{P}$ :

$$
0 \rightarrow \omega_{0}^{\kappa} \rightarrow \omega^{\kappa} \stackrel{\text { res }}{\rightarrow} \Omega_{\mathrm{P}}^{\kappa} \rightarrow 0
$$

\section{La partie ordinaire}

\subsection{Définition des opérateurs de Hecke}

5.1.1. Algèbres de Hecke. - On rappelle que pour $j=0, \ldots, g-1$ nous avons posés $\alpha_{j}=\left(\begin{array}{cc}1_{g-j} & 0 \\ 0 & p \cdot 1_{j}\end{array}\right) \in \mathrm{GL}_{g}\left(\mathbb{Q}_{p}\right)$. Notons $\alpha_{j}^{\prime}=\alpha_{j}^{-1} \cdot p$.

Pour $j=1, \ldots, g-1$ nous posons $\beta_{j}=\left(\begin{array}{cc}\alpha_{j} & 0 \\ 0 & \omega_{0} \alpha_{j}^{-1} \omega_{0} \cdot p^{2}\end{array}\right) \in \mathrm{G}\left(\mathbb{Q}_{p}\right)$ et également $\beta_{0}=\left(\begin{array}{cc}1_{g} & 0 \\ 0 & p \cdot 1_{g}\end{array}\right)$.

Soit $\Sigma_{g}$ le groupe des matrices symétriques de taille $g \times g$. Définissons les entiers

$$
m_{j}=\sharp \Sigma_{g}(\mathbb{Z}) / \alpha_{j}^{\prime} \Sigma_{g}(\mathbb{Z}) \alpha_{j}^{\prime} .
$$

Soit $\mathrm{P}$ un parabolique standard de $\mathrm{GL}_{g}$ associé à des entiers strictement positifs $n_{1}, \ldots, n_{r}$ vérifiant $\sum_{i=1}^{r} n_{i}=g$. Rappelons que $\mathrm{S}$ est le sous-groupe parabolique de Siegel de G. Ses sous-groupes $\mathrm{S}_{\mathrm{P}}$ et $\mathrm{S}_{S \mathrm{P}}$ sont définis dans la section 2. Considérons les groupes de congruence $K_{\mathrm{P}}(n)=\left\{M \in \mathrm{S}\left(\mathbb{Z}_{p}\right), M \in\right.$ 
$\left.\mathrm{S}_{\mathrm{P}} \bmod p^{n}\right\}$ et $K_{S \mathrm{P}}(n)=\left\{M \in \mathrm{S}\left(\mathbb{Z}_{p}\right), M \in \mathrm{S}_{S \mathrm{P}} \bmod p^{n}\right\}$. On leur associe les algèbres de Hecke non commutatives

$$
\mathcal{H}\left(\mathrm{G}\left(\mathbb{Q}_{p}\right) / / K_{\mathrm{P}}(n)\right) \text { et } \mathcal{H}\left(\mathrm{G}\left(\mathbb{Q}_{p}\right) / / K_{S \mathrm{P}}(n)\right)
$$

des fonctions $K_{\mathrm{P}}(n)$ et $K_{S \mathrm{P}}(n)$ bi-invariantes sur $\mathrm{G}\left(\mathbb{Q}_{p}\right)$, à support compact, à valeur dans $\mathbb{Z}$, munies du produit de convolution.

Rappelons qu'on a posé, pour $1 \leq l \leq r, N_{l}=\sum_{i=1}^{l} n_{i}$. Posons également $N_{0}=0$. Soit $\mathbb{H}_{\mathrm{P}} \subset \mathrm{T}_{\mathrm{G}}\left(\mathbb{Q}_{p}\right)$ le monoïde engendré par les matrices $\left(\beta_{N_{l}}\right)_{0 \leq l \leq r-1}$. Tout $\beta \in \mathbb{H}_{\mathrm{P}}$ s'écrit de manière unique $\prod_{l=0}^{r-1} \beta_{N_{l}}^{t_{l}}$ avec $t_{l} \in \mathbb{N}$. On pose $m_{\beta}=$ $\prod_{l=0}^{r-1} m_{N_{l}}^{t_{l}}$.

Pour $n \geq 1$, les applications $\beta \mapsto K_{\mathrm{P}}(n) \beta K_{\mathrm{P}}(n)$ et $\beta \mapsto K_{S \mathrm{P}}(n) \beta K_{S \mathrm{P}}(n)$ permettent d'identifier $\mathbb{Z}\left[\mathbb{H}_{\mathrm{P}}\right]$ avec des sous-algèbres commutatives des algèbres de Hecke $\mathscr{H}\left(\mathrm{G}\left(\mathbb{Q}_{p}\right) / / K_{\mathrm{P}}(n)\right)$ et $\mathcal{H}\left(\mathrm{G}\left(\mathbb{Q}_{p}\right) / / K_{S \mathrm{P}}(n)\right)$ (voir [7] 3.6).

À chaque élément $\beta \in \mathbb{H}_{\mathrm{P}}$, on va maintenant associer des correspondances et des opérateurs de Hecke.

5.1.2. Espaces rigides associés à la tour d'Igusa, modification de la struture entière. - À cause de la remarque 3.2, les opérateurs de Hecke que nous allons définir ne stabiliseront pas la structure entière définie par le faisceau $\omega^{\kappa}$. Pour cette raison, nous devons également travailler au niveau rigide.

Soit $\mathrm{P}$ un parabolique standard de $\mathrm{GL}_{g}$ et $\mathrm{Q}=\omega_{0} \cdot{ }^{t} \mathrm{P} \cdot \omega_{0}$.

Notons $\phi_{\text {rig }}$ l'espace rigide associé au schéma formel $\phi_{\infty}$ (voir [2]). Pour tout $n \in \mathbb{N}_{\geq 1}$, on note $\mathcal{J}_{\text {rig, } n} / \mathrm{P}$ l' espace rigide associé au schéma formel $\mathcal{J}_{\infty, n} / \mathrm{P}$. Pour tout poids $\kappa \in X\left(\mathrm{~T}_{\mathrm{Q}}\right)$ on note $\omega_{\text {rig }}^{\kappa}$ le faisceau cohérent rigide associé à $\omega^{\kappa}$. Comme ces espaces rigides sont quasi-compacts, on a $\mathrm{H}^{0}\left(\phi_{\text {rig }}, \omega_{\text {rig }}^{\kappa}\right)=$ $\mathrm{H}^{0}\left(\phi_{\infty}, \omega^{\kappa}\right) \otimes \mathbb{Q}_{p}$ et $\mathrm{H}^{0}\left(\mathcal{J}_{\text {rig }, n} / \mathrm{P}, \omega_{\text {rig }}^{\kappa}\right)=\mathrm{H}^{0}\left(\mathcal{J}_{\infty, n} / \mathrm{P}, \omega^{\kappa}\right) \otimes \mathbb{Q}_{p}$.

Soit $\mathcal{J}_{\infty} / S \mathrm{P} \stackrel{f}{\rightarrow} \phi_{\infty}$ le morphisme d'oubli. Notons $\omega^{\text {top }, \mathrm{P}, \kappa}=f_{\star} \vartheta_{\mathcal{J}_{\infty, \infty} / S \mathrm{P}}\left[-\kappa^{\prime}\right]$. Notons $\omega_{\text {rig }}^{\text {top }, \kappa}$ le faisceau rigide associé. On dispose de morphismes $\Theta_{\mathrm{P}}^{\star}: \omega^{\kappa} \rightarrow \omega^{\text {top }, \mathrm{P}, \kappa}(\operatorname{voir} 4.2 .1)$.

$\mathrm{Au}$ dessus du morphisme d'espaces annelés $\phi_{\text {rig }} \rightarrow \phi_{\infty}$, on a un diagramme commutatif :

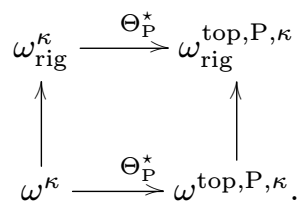


Ce diagramme est l'analogue relatif du diagramme (voir le numero 3.1.2)

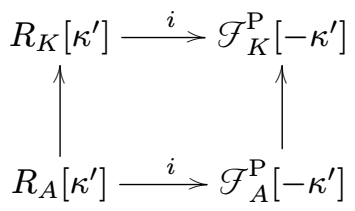

Considérons le faiscau de $\theta_{\phi_{\infty}}$-modules $\tilde{\omega}^{\kappa}=\omega_{\text {rig }}^{\kappa} \times{ }_{\omega_{\text {rig }}^{\text {top }, \kappa}, \Theta_{\mathrm{P}}^{\star}} \omega^{\text {top }, \mathrm{P}, \kappa}$. C'est la modification de structure entière qui sera stable sous l'action des opérateurs de Hecke. Le faisceau $\tilde{\omega}^{\kappa}$ est l'analogue relatif du module $\tilde{R}_{A}\left[\kappa^{\prime}\right]$ de 3.1.2.

On considère aussi le pro-espace rigide analytique $\mathcal{T}_{\text {rig, }, \infty} / \mathrm{P}$, limite projective des $\mathcal{J}_{\text {rig, }, n} / \mathrm{P}$. On dispose d'une suite exacte

$$
0 \rightarrow \omega_{0, \text { rig }}^{\kappa} \rightarrow \omega_{\text {rig }}^{\kappa} \stackrel{\text { res }}{\rightarrow} \Omega_{\mathrm{P}, \text { rig }}^{\kappa} \rightarrow 0
$$

de faisceaux cohérents sur $\mathcal{J}_{\text {rig, } \infty} / \mathrm{P}$ déduite de la suite exacte du numéro 4.2.2.

5.1.3. Les opérateur $T_{\beta}^{1}$ agissant sur les formes modulaires. - Soit $\mathcal{J}_{\infty, n}^{\mathrm{nc}} / \mathrm{P}$ l'ouvert modulaire de $\mathcal{J}_{\infty, n} / \mathrm{P}$ complémentaire du diviseur à l'infini. Prendre la partie «non compactifiée » va nous permettre de définir les correspondances de façon modulaire.

REMARQUE 5.1. - Il est possible que des travaux généralisant [21] permettent de « compactifier» les correspondances que nous allons définir.

Soit $\beta \in \mathbb{H}_{\mathrm{P}}$. La double classe $K_{\mathrm{P}}(n) \beta K_{\mathrm{P}}(n)$ nous permet de sélectionner une composante du schéma des $p$-isogénies au dessus de $\mathcal{J}_{\infty, n} / \mathrm{P}$ dans l'esprit de [3], chapitre VII. Précisément :

On a $\beta=\operatorname{diag}\left(\alpha, \nu(\beta) \omega_{0} \alpha^{-1} \omega_{0}\right)$. Considérons la correspondance $\mathcal{T}_{\infty, n}^{\mathrm{nc}} / \mathrm{P}(\beta)$ dont les $R$-points sont la donnée de :

- Une variété abélienne ordinaire principalement polarisée $\mathscr{G} \stackrel{f}{\rightarrow}$ Spec $R$ et d'un P-drapeau : $\psi_{n}: \mu_{p^{n}}^{g} \simeq \mathscr{C}\left[p^{n}\right]^{0} \bmod \mathrm{P}$.

- Une variété abélienne ordinaire principalement polarisée $\mathscr{G}^{\prime} \stackrel{f^{\prime}}{\rightarrow}$ Spec $R$ et d'un P-drapeau : $\psi_{n}^{\prime}: \mu_{p^{n}}^{g} \simeq \mathscr{G}^{\prime}\left[p^{n}\right]^{0} \bmod \mathrm{P}$.

- Une $p$-isogénie de facteur de similitude $\nu(\beta), \pi: \mathscr{C} \rightarrow \mathscr{C}^{\prime}$ telle qu'il existe (localement pour la topologie étale) des trivialisations $\psi_{\infty}: \mu_{p^{\infty}}^{g} \simeq$ $\mathscr{G}\left[p^{\infty}\right]^{0},\left.\psi_{\infty}\right|_{\mu_{p^{n}}^{g}} \bmod \mathrm{P}=\psi_{n}$ et $\psi_{\infty}^{\prime}: \mu_{p^{\infty}}^{g} \simeq \mathscr{G}\left[p^{\infty}\right]^{0},\left.\psi_{\infty}^{\prime}\right|_{\mu_{p^{n}}^{g}} \bmod \mathrm{P}=$ $\psi_{n}^{\prime}$ pour lesquelles

$$
\psi_{\infty}^{\prime-1} \circ \pi \circ \psi_{\infty}=\alpha
$$

TOME $140-2012-\mathrm{N}^{\mathrm{O}} 3$ 
On dispose de deux projections : $p_{1}$ induite par l'oubli de $\mathscr{G}^{\prime}$ et $\pi, p_{2}$ induite par l'oubli de $\mathscr{C}$ et $\pi$.

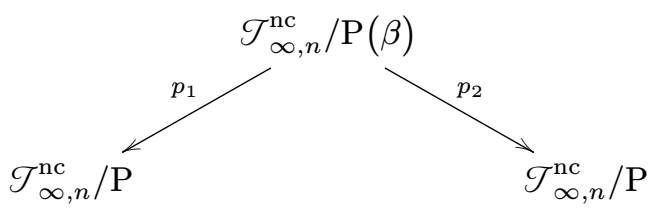

5.1.3.1. Le morphisme $\pi_{\beta}^{\kappa}$. — À l'aide du morphisme

$$
\pi^{\star}: e^{\star} \Omega_{Q^{\prime} /\left(\mathcal{T}_{\infty, n}^{\mathrm{nc}} / \mathrm{P}(\beta)\right)}^{1} \rightarrow e^{\star} \Omega_{\mathscr{Q} /\left(\mathcal{J}_{\infty, n}^{\mathrm{nc}} / \mathrm{P}(\beta)\right)}^{1}
$$

on construit un morphisme au niveau des espaces rigides $\pi_{\beta}^{\kappa}: p_{2}^{\star} \omega_{\text {rig }}^{\kappa} \rightarrow p_{1}^{\star} \omega_{\text {rig }}^{\kappa}$. On verra que ce morphisme induit un morphisme $\pi_{\beta}^{\kappa}: p_{2}^{\star} \tilde{\omega}^{\kappa} \rightarrow p_{1}^{\star} \tilde{\omega}^{\kappa}$.

Sur un ouvert affinoïde $\operatorname{Spm} K$ de $\mathcal{T}_{\text {rig, }, n}^{\text {nc }} / \mathrm{P}(\beta)$, on a

$$
\begin{aligned}
\pi_{\beta}^{\kappa}: p_{2}^{\star} \omega_{\text {rig }}^{\kappa}(K) & \rightarrow p_{1}^{\star} \omega_{\text {rig }}^{\kappa}(K) \\
f & \mapsto\left[\pi_{\beta}^{\kappa}(f): \omega \mapsto \kappa^{\prime}(\alpha) \cdot f\left(\omega^{\prime}\right)\right]
\end{aligned}
$$

où $\omega: K^{g} \simeq e^{\star} \Omega_{\mathscr{C} / K}^{1}$ et $\omega^{\prime}: K^{g} \simeq e^{\star} \Omega_{\mathscr{G}^{\prime} / K}^{1}$ sont des trivialisations des faisceaux conormaux vérifiant : $\pi^{\star} \omega^{\prime}=\omega$. Les éléments de $p_{i}^{\star} \omega^{\kappa}(K), i \in\{1,2\}$, sont vus comme des fonctions sur les trivialisations des faisceaux conormaux.

Soit $\bar{x} \in\left(\mathcal{T}_{\infty, n}^{\mathrm{nc}} / \mathrm{P}(\beta)\right)\left(\overline{\mathbb{F}}_{p}\right)$. Posons $B=\mathscr{\vartheta}_{\mathcal{T}_{\infty, n}^{\mathrm{nc}} / \mathrm{P}(\beta), \bar{x}}$ le hensélisé strict de $\mathcal{J}_{\infty, n}^{\mathrm{nc}} / \mathrm{P}(\beta)$ en $\bar{x}$ et posons $K=B[1 / p]$.

Le lemme suivant fait le lien avec le numéro 3.2.

LEMme 5.1. - Il existe un diagramme commutatif

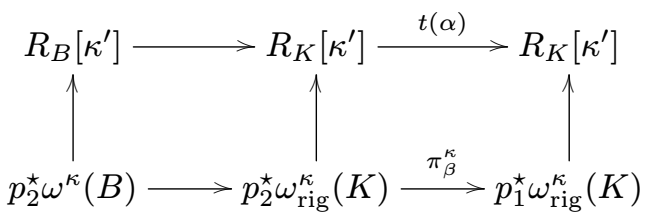

où les applications verticales sont des isomorphismes. De plus, lorsque $n=\infty$, le diagramme est compatibles avec les applications res.

Démonstration. - Au dessus de Spec $B$, on dispose des deux schémas abéliens $\mathscr{G}$ et $\mathscr{G}^{\prime}$ ainsi que des trivialisations $\psi_{\infty}: \mu_{p^{\infty}}^{g} \simeq \mathscr{G}\left[p^{\infty}\right]^{0}, \psi_{\infty}^{\prime}: \mu_{p^{\infty}}^{g} \simeq \mathscr{G}^{\prime}\left[p^{\infty}\right]^{0}$ compatibles avec les trivialisations partielles $\psi_{n}$ et $\psi_{n}^{\prime}$. L'isogénie $\pi$ est donc 
donnée par $\alpha$ sur la partie connexe des groupes $p$-divisibles et le diagramme suivant commute :

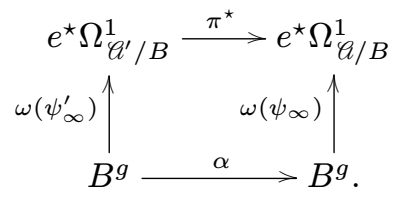

À toute section $f$ de $p_{1}^{\star} \omega^{\kappa}$ (resp. $\left.p_{2}^{\star} \omega^{\kappa}\right)$, on associe un élément $\tilde{f} \in R_{B}[\kappa]$, par la formule $\tilde{f}(g)=f\left(\omega\left(\psi_{\infty}\right) \circ g\right)$ (resp. $\left.\tilde{f}(g)=f\left(\omega\left(\psi_{\infty}^{\prime}\right) \circ g\right)\right)$. On a alors

$$
\begin{aligned}
\tilde{\pi_{\beta}^{\kappa} f(g)} & =\pi_{\beta}^{\kappa} f\left(\omega\left(\psi_{\infty}\right) \circ g\right) \\
& =f\left(\left(\pi^{\star}\right)^{-1} \omega\left(\psi_{\infty}\right) \circ g \circ \alpha\right) \\
& =f\left(\omega\left(\psi_{\infty}^{\prime}\right) \circ \alpha^{-1} \circ g \circ \alpha\right) \\
& =\tilde{f}\left(\alpha^{-1} g \alpha\right) \\
& =t(\alpha) \tilde{f}(g)
\end{aligned}
$$

La compatibilité aux morphismes res est claire.

Le corollaire qui suit résulte alors du lemme précédent et de la proposition 3.1 .

Corollaire 5.1. - Le morphisme $\pi_{\beta}^{\kappa}$ induit un morphisme $\pi_{\beta}^{\kappa}: p_{2}^{\star} \tilde{\omega}^{\kappa} \rightarrow$ $p_{1}^{\star} \tilde{\omega}^{\kappa}$.

On obtient également, lorsque $n=\infty$, des morphismes (notés de la même manière) $\pi_{\beta}^{\kappa}: p_{2}^{\star} \omega_{0, \text { rig }}^{\kappa} \rightarrow p_{1}^{\star} \omega_{0, \text { rig }}^{\kappa} \pi_{\beta}^{\kappa}: p_{2}^{\star} \Omega_{\mathrm{P}, \text { rig }}^{\kappa} \rightarrow p_{1}^{\star} \Omega_{\mathrm{P}, \text { rig }}^{\kappa}$. Cette dernière application provient d'ailleurs d'une application $\pi_{\beta}^{\kappa}: p_{2}^{\star} \Omega_{\mathrm{P}}^{\kappa} \rightarrow p_{1}^{\star} \Omega_{\mathrm{P}}^{\kappa}$ définie au niveau des schémas formels.

5.1.3.2. Le morphisme trace. - On dispose d'une application de trace normalisée

$$
\operatorname{Tr}_{\beta}=m_{\beta}^{-1} \operatorname{Tr}_{p_{1}}:\left(p_{1}\right)_{\star} \vartheta_{\mathcal{T}_{\infty, n}^{\mathrm{nc}} / \mathrm{P}(\beta)} \rightarrow \Theta_{\mathcal{T}_{\infty, n}^{\mathrm{nc}} / \mathrm{P}}
$$

L'entier $m_{\beta}$ correspond au degré d'inséparabilité de la projection $p_{1}$ par la théorie de Serre-Tate ([11]) et en dépit de la normalisation, l'application est bien définie. On renvoie au corollaire A.1 de l'appendice pour plus de détails.

5.1.3.3. L'opérateur $T_{\beta}^{1}$. - $\mathrm{Si} \mathcal{F}$ est l' un des trois faisceaux $\omega_{\text {rig }}^{\kappa}, \Omega_{\mathrm{P}, \text { rig }}^{\kappa}, \omega_{0, \text { rig }}^{\kappa}$ (les deux derniers n'étant définis que sous l'hypothèse $n=\infty$ ), on peut considérer le morphisme composé :

$$
\begin{aligned}
T_{\beta}^{1}: \mathrm{H}^{0}\left(\mathcal{J}_{\text {rig, }, n}^{\mathrm{nc}} / \mathrm{P}, \mathcal{F}\right) \rightarrow \mathrm{H}^{0} & \left(\mathcal{J}_{\text {rig }, n}^{\mathrm{nc}} / \mathrm{P}(\beta), p_{2}^{\star} \mathcal{F}\right) \\
& \stackrel{\pi_{\beta}^{\kappa}}{\rightarrow} \mathrm{H}^{0}\left(\mathcal{J}_{\text {rig }, n}^{\mathrm{nc}} / \mathrm{P}(\beta), p_{1}^{\star} \mathcal{F}\right) \stackrel{\operatorname{Tr}_{\beta}}{\rightarrow} \mathrm{H}^{0}\left(\mathcal{J}_{\text {rig }, n}^{\mathrm{nc}} / \mathrm{P}, \mathcal{F}\right)
\end{aligned}
$$

TOME $140-2012-\mathrm{N}^{\mathrm{O}} 3$ 
Ce morphisme induit également des morphismes :

$$
T_{\beta}^{1}: \mathrm{H}^{0}\left(\mathcal{T}_{\infty, n}, \tilde{\omega}^{\kappa}\right) \rightarrow \mathrm{H}^{0}\left(\mathcal{T}_{\infty, n}, \tilde{\omega}^{\kappa}\right) .
$$

De façon concrète, on a la formule habituelle :

$$
\left.f\right|_{T_{\beta}^{1}}\left(\mathscr{G}, \psi_{\mathcal{N}}, \psi_{\infty} \bmod \mathrm{P}, \omega\right)=\kappa^{\prime}(\alpha) \cdot p^{-m_{\beta}} \sum_{\mathscr{G}^{\stackrel{\pi}{\rightarrow}} \mathscr{C}^{\prime}, \text { de type } \beta} f\left(\mathscr{G}^{\prime}, \psi_{\mathcal{N}}^{\prime}, \psi_{\infty}^{\prime} \bmod \mathrm{P}, \omega^{\prime}\right) .
$$

La somme porte sur toutes les isogénies de type $K_{\mathrm{P}}(n) \beta K_{\mathrm{P}}(n)$ et $\pi^{\star} \omega^{\prime}=\omega$.

REMARQUe 5.2. - L'opérateur $T_{\beta_{0}}^{1}$ est souvent noté $U_{p}$.

Cette formule permet donc de calculer «physiquement» l'action de la correspondance. En conséquence, le formalisme habituel des opérations sur les doubles classes s'applique.

En particulier, comme la décomposition des doubles classes $K_{\mathrm{P}}(n) \beta K_{\mathrm{P}}(n)$ est indépendante de $n \geq 1$, l'action est compatible avec le changement de niveau.

D'après le principe de Koecher, $\mathrm{H}^{0}\left(\mathcal{J}_{\text {rig,n } n}^{\text {nc }} / \mathrm{P}, \mathcal{F}\right)=\mathrm{H}^{0}\left(\mathcal{J}_{\text {rig }, n} / \mathrm{P}, \mathcal{F}\right)$. L'endomorphisme $T_{\beta}^{1}$ agit donc sur $\mathrm{H}^{0}\left(\mathcal{J}_{\text {rig }, n} / \mathrm{P}, \mathcal{F}\right)$ et il envoie la partie cuspidale sur la partie cuspidale (car une somme de formes cuspidales est cuspidale). En résumé :

Proposition 5.1. - Pour tout $n \in \mathbb{N}_{\geq 1}$, l'application $\beta \mapsto T_{\beta}^{1}$ définit des morphismes d'algèbres

$$
\mathbb{Z}\left[\mathbb{H}_{\mathrm{P}}\right] \rightarrow \operatorname{End}\left(\mathrm{H}^{0}\left(\mathcal{J}_{\text {rig }, n} / \mathrm{P}, \omega^{\kappa}\right)\right) \text { et } \mathbb{Z}\left[\mathbb{H}_{\mathrm{P}}\right] \rightarrow \operatorname{End}\left(\mathrm{H}^{0}\left(\mathcal{J}_{\text {rig }, \infty} / \mathrm{P}, \Omega_{\mathrm{P}, \text { rig }}^{\kappa}\right)\right) \text {. }
$$

L'action de $\mathbb{Z}\left[\mathbb{H}_{\mathrm{P}}\right]$ respecte l'inclusion $\mathrm{H}^{0}\left(\mathcal{J}_{\infty, n} / \mathrm{P}, \tilde{\omega}^{\kappa}\right) \hookrightarrow \mathrm{H}^{0}\left(\mathcal{J}_{\text {rig }, n} / \mathrm{P}, \omega_{\text {rig }}^{\kappa}\right)$, les formes cuspidales et est compatible avec les morphismes :

$-\mathrm{H}^{0}\left(\mathcal{J}_{\text {rig }, n} / \mathrm{P}, \omega^{\kappa}\right) \rightarrow \mathrm{H}^{0}\left(\mathcal{J}_{\text {rig }, n+1} / \mathrm{P}, \omega^{\kappa}\right)$.

- res : $\mathrm{H}^{0}\left(\mathcal{J}_{\text {rig }, \infty} / \mathrm{P}, \omega^{\kappa}\right) \rightarrow \mathrm{H}^{0}\left(\mathcal{J}_{\text {rig }, \infty} / \mathrm{P}, \Omega_{\mathrm{P}}^{\kappa}\right)$.

5.1.4. L'opérateur $T_{\beta}^{1}$ agissant sur les formes p-adiques. - Soit $\beta \in \mathbb{H}_{\mathrm{P}}$, $\beta=\operatorname{diag}\left(\alpha, \nu(\beta) \omega_{0} \alpha^{-1} \omega_{0}\right)$. La double classe $K_{S \mathrm{P}}(n) \beta K_{S \mathrm{P}}(n)$ définit une correspondance $\mathcal{J}_{\infty, n}^{\text {nc }} / S \mathrm{P}(\beta)$ dont les $R$-points sont la donnée de :

- Une variété abélienne ordinaire principalement polarisée $\mathscr{G} \stackrel{f}{\rightarrow} R$ et d'un $S$ P-drapeau : $\psi_{n}: \mu_{p^{n}}^{g} \simeq \mathscr{C}\left[p^{n}\right]^{0} \bmod S \mathrm{P}$.

- Une variété abélienne ordinaire principalement polarisée $\mathscr{G}^{\prime} \stackrel{f^{\prime}}{\rightarrow} R$ et d'un $S$ P-drapeau : $\psi_{n}: \mu_{p^{n}}^{g} \simeq \mathscr{C}\left[p^{n}\right]^{0} \bmod S \mathrm{P}$. 
- Une $p$-isogénie de facteur de similitude $\nu(\beta), \pi: \mathscr{G} \rightarrow \mathscr{Q}^{\prime}$ telle qu'il existe (localement pour la topologie étale) des trivialisations $\psi_{\infty}: \mu_{p^{\infty}}^{g} \simeq \mathscr{C}\left[p^{\infty}\right]^{0},\left.\psi_{\infty}\right|_{\mu_{p^{n}}^{g}} \bmod S \mathrm{P}=\psi_{n}$ et $\psi_{\infty}^{\prime}: \mu_{p^{\infty}}^{g} \simeq$ $\mathscr{C}\left[p^{\infty}\right]^{0},\left.\psi_{\infty}^{\prime}\right|_{\mu_{p^{n}}^{g}} \bmod S \mathrm{P}=\psi_{n}^{\prime}$ pour lesquelles

$$
\psi_{\infty}^{\prime-1} \circ \pi \circ \psi_{\infty}=\alpha
$$

On dispose à nouveau de deux projections $p_{1}, p_{2}: \mathcal{T}_{\infty, n}^{\mathrm{nc}} / S \mathrm{P}(\beta) \rightarrow \mathcal{T}_{\infty, n} / S \mathrm{P}$ et donc d'opérateurs $T_{\beta}^{1}$ agissant sur $V_{\infty, n}^{S \mathrm{P}}=\mathrm{H}^{0}\left(\mathcal{T}_{\infty, n}^{\mathrm{nc}} / S \mathrm{P}, \vartheta_{\mathcal{T}_{\infty, n} / S \mathrm{P}}\right)$ (principe de Koecher) par la composition

$$
T_{\beta}^{1}: V_{\infty, n}^{S \mathrm{P}} \stackrel{p_{2}^{\star}}{\longrightarrow} \mathrm{H}^{0}\left(\mathcal{T}_{\infty, n}^{\mathrm{nc}} / S \mathrm{P}(\beta), \vartheta_{\mathcal{T}_{\infty, n} / S \mathrm{P}(\beta)}\right) \stackrel{\operatorname{Tr}_{\beta}}{\longrightarrow} V_{\infty, n}^{S \mathrm{P}}
$$

Proposition 5.2. - L'application $\beta \mapsto T_{\beta}^{1}$ définit un morphisme d'algèbres

$$
\mathbb{Z}\left[\mathbb{H}_{\mathrm{P}}\right] \rightarrow \operatorname{End}\left(V_{m, n}^{S \mathrm{P}}\right) .
$$

L'action de $\mathbb{Z}\left[\mathbb{H}_{\mathrm{P}}\right]$ respecte les formes cuspidales et est compatible avec les morphismes :

$$
\begin{aligned}
& -V_{\infty, n}^{S \mathrm{P}} \rightarrow V_{\infty, n+1}^{S \mathrm{P}} . \\
& -\Lambda_{\infty, \infty}: \mathrm{H}^{0}\left(\mathcal{J}_{\infty} / \mathrm{P}, \Omega_{\mathrm{P}}^{\kappa}\right) \tilde{\rightarrow} V_{\infty}^{S \mathrm{P}}\left[-\kappa^{\prime}\right] .
\end{aligned}
$$

Démonstration. - Vérifions l'équivariance du morphisme

$$
\Lambda_{\infty, \infty}: \mathrm{H}^{0}\left(\mathcal{T}_{\infty} / \mathrm{P}, \Omega_{\mathrm{P}}^{\kappa}\right) \rightarrow V_{\infty}^{S \mathrm{P}}\left[-\kappa^{\prime}\right] .
$$

Soit $h: \mathcal{J}_{\infty} / S \mathrm{P} \rightarrow \mathcal{J}_{\infty} / \mathrm{P}$ et $h^{\prime}: \mathcal{J}_{\infty} / S \mathrm{P}(\beta) \rightarrow \mathcal{T}_{\infty} / \mathrm{P}(\beta)$ les projections. On dispose d'un morphisme de faisceaux sur $\mathcal{T}_{\infty} / \mathrm{P}, \Omega_{\mathrm{P}}^{\kappa} \rightarrow h_{\star} \vartheta_{\mathcal{T}_{\infty} / S \mathrm{P}}$. Ce dernier morphisme induit, pour $i \in\{1,2\}$, des morphismes de $\mathcal{T}_{\infty}^{\text {nc }} / \mathrm{P}(\beta)$-faisceaux

$$
p_{i}^{\star} \Omega^{\kappa} \rightarrow h_{\star}^{\prime} \theta_{T_{\infty, \infty}^{\mathrm{nc}} / S \mathrm{P}(\beta)} .
$$

Il s'agit alors de vérifier que le diagramme suivant commute :

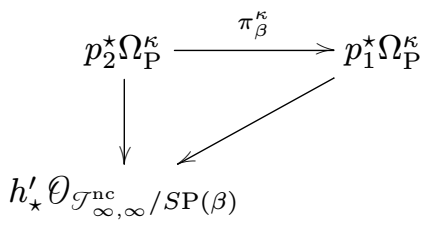

La vérification est locale pour la topologie étale, reprenons les notations de la démonstration du lemme 5.1. La formule

$$
\pi_{\beta}^{\kappa} f\left(\omega\left(\psi_{\infty}\right) \circ g\right)=f\left(\left(\pi^{\star}\right)^{-1} \omega\left(\psi_{\infty}\right) \circ g \circ \alpha\right)=f\left(\omega\left(\psi_{\infty}^{\prime}\right) \circ \alpha_{j}^{-1} \circ g \circ \alpha\right)
$$

appliquée avec $g=1$ donne la commutativité et en revenant aux définitions on en déduit les compatibilités annoncées. 


\subsection{Propriétés des opérateurs de Hecke}

5.2.1. Contraction. - Posons $\beta_{\mathrm{P}}=\prod_{l=0}^{r-1} \beta_{N_{l}}$. L'opérateur $T_{\beta_{\mathrm{P}}}^{1}$ diminue le niveau en $p$ dans le sens suivant (voir aussi [7], p. 39) :

Proposition 5.3. - Supposons $n \geq 2$, alors :

$$
T_{\beta_{\mathrm{P}}}^{1} \cdot \mathrm{H}^{0}\left(\mathcal{J}_{\text {rig }, n} / \mathrm{P}, \omega_{\text {rig }}^{\kappa}\right) \subset \mathrm{H}^{0}\left(\mathcal{J}_{\text {rig }, n-1} / \mathrm{P}, \omega_{\text {rig }} \operatorname{rig} \kappa\right) .
$$

Démonstration. - On va vérifier que $K_{\mathrm{P}}(n) \beta_{\mathrm{P}} K_{\mathrm{P}}(n)=K_{\mathrm{P}}(n) \beta_{\mathrm{P}} K_{\mathrm{P}}(n-1)$. Posons $\beta_{\mathrm{P}}^{-1} K_{\mathrm{P}}(n) \beta_{\mathrm{P}} \cap K_{\mathrm{P}}(n)=K_{\mathrm{P}}(n)^{\beta_{\mathrm{P}}}$ et $\beta_{\mathrm{P}}^{-1} K_{\mathrm{P}}(n) \beta_{\mathrm{P}} \cap K_{\mathrm{P}}(n-1)=$ $K_{\mathrm{P}}(n-1)^{\beta_{\mathrm{P}}}$ pour alléger les notations. Comme on sait que $K_{\mathrm{P}}(n) \beta_{\mathrm{P}} K_{\mathrm{P}}(n)=$ $\cup_{u} K_{\mathrm{P}}(n) \beta_{\mathrm{P}} u$, où $u$ est un système de représentants de $K_{\mathrm{P}}(n)^{\beta_{\mathrm{P}}} \backslash K_{\mathrm{P}}(n)$ et $K_{\mathrm{P}}(n) \beta_{\mathrm{P}} K_{\mathrm{P}}(n-1)=\cup_{u^{\prime}} K_{\mathrm{P}}(n) \beta_{\mathrm{P}} u^{\prime}$, où $u^{\prime}$ est un système de représentants de $K_{\mathrm{P}}(n-1)^{\beta_{\mathrm{P}}} \backslash K_{\mathrm{P}}(n-1)$, on est amené à vérifier que le morphisme naturel $K_{\mathrm{P}}(n)^{\beta_{\mathrm{P}}} \backslash K_{\mathrm{P}}(n) \rightarrow K_{\mathrm{P}}(n-1)^{\beta_{\mathrm{P}}} \backslash K_{\mathrm{P}}(n-1)$ est un isomorphisme. Considérons alors le diagramme commutatif

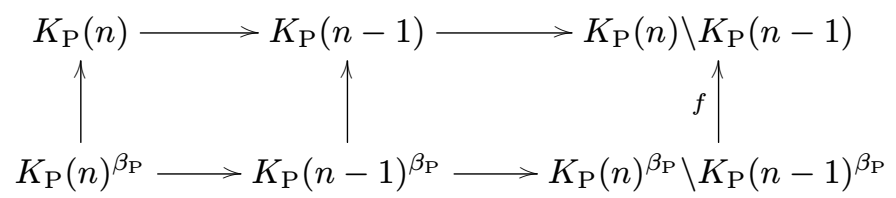

Les lignes sont des suites exactes longues. En appliquant le lemme du serpent, on se ramène à vérifier que le morphisme vertical $f$ est un isomorphisme. Soit $\mathrm{U}_{\mathrm{P}}$ le radical unipotent de $\mathrm{P}$. En explicitant les groupes on vérifie que la source et le but de $f$ sont isomorphes au noyau $\operatorname{Ker}\left(\mathrm{U}_{\mathrm{P}}^{0}\left(\mathbb{Z} / p^{n}\right) \rightarrow U_{\mathrm{P}}^{0}\left(\mathbb{Z} / p^{n-1}\right)\right) \mathrm{du}$ morphisme de réduction modulo $p^{n-1}$.

\subsubsection{Le projecteur}

DÉFInition 5.1. - On définit un projecteur $e_{\mathrm{P}}$ de $\mathrm{P}$-ordinarité par la formule :

$$
e_{\mathrm{P}}=\lim _{n \rightarrow \infty}\left(T_{\beta_{\mathrm{P}}}^{1}\right)^{n !}
$$

L'algèbre des formes p-adiques $\mathrm{P}$-ordinaires est l'image $e_{\mathrm{P}} V_{\infty}^{S \mathrm{P}}$ du projecteur.

Pour tout poids $\kappa \in X\left(\mathrm{~T}_{\mathrm{Q}}\right)$, on note $\mathrm{M}^{\text {ord-P }}\left(\kappa, \Gamma_{\mathcal{N}}, \mathbb{Z}_{p}\right)$ et $\mathrm{S}^{\text {ord-P }}\left(\kappa, \Gamma_{\mathcal{N}}, \mathbb{Z}_{p}\right)$ les images de $\mathrm{M}\left(\kappa, \Gamma_{\mathcal{N}}, \mathbb{Z}_{p}\right)$ et $\mathrm{S}\left(\kappa, \Gamma_{\mathcal{N}}, \mathbb{Z}_{p}\right)$ dans $e_{\mathrm{P}} V_{\infty}^{S \mathrm{P}}$.

REMARQUE 5.3. - Le projecteur $e_{\mathrm{P}}$ ne respecte pas les modules $\mathrm{M}\left(\kappa, \Gamma_{\mathcal{N}}, \mathbb{Z}_{p}\right)$ et $\mathrm{S}\left(\kappa, \Gamma_{\mathcal{N}}, \mathbb{Z}_{p}\right)$. Les éléments de $\mathrm{M}^{\text {ord-P }}\left(\kappa, \Gamma_{\mathcal{N}}, \mathbb{Z}_{p}\right)$ et $\mathrm{S}^{\text {ord-P }}\left(\kappa, \Gamma_{\mathcal{N}}, \mathbb{Z}_{p}\right)$ sont des formes modulaires de niveau $\Gamma_{\mathcal{N}} \cap \mathrm{I}_{\mathrm{P}}$, où $\mathrm{I}_{\mathrm{P}}$ est le sous-groupe parahorique de $\mathrm{G}(\mathbb{Z})$ des matrices dont la réduction modulo $p$ appartient à $\mathrm{S}_{\mathrm{P}}(\mathbb{Z} / p \mathbb{Z})$. 
Proposition 5.4. - Pour tout $\kappa \in X\left(\mathrm{~T}_{\mathrm{Q}}\right)^{+}$, on a des isomorphismes

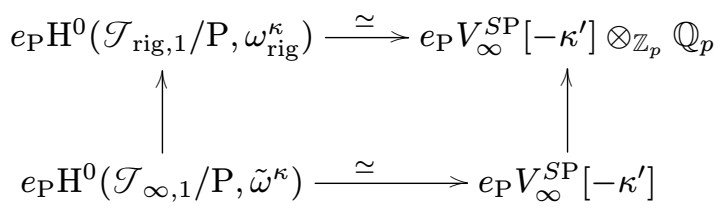

Démonstration. - Considérons le diagramme suivant (le morphisme res est défini au numéro 5.1.2, l'application $\Lambda_{\infty, \infty}$ au numéro 4.2.2) :

$$
\begin{aligned}
& \mathrm{H}^{0}\left(\mathcal{J}_{\text {rig }, \infty} / \mathrm{P}, \omega_{\text {rig }}^{\kappa}\right) \stackrel{\text { res }}{\longrightarrow} \mathrm{H}^{0}\left(\mathcal{J}_{\text {rig }, \infty} / \mathrm{P}, \Omega_{\mathrm{P}, \text { rig }}^{\kappa}\right) \stackrel{\Lambda_{\infty, \infty}^{\text {rig }}}{\longrightarrow} V_{\infty}^{S \mathrm{P}}\left[-\kappa^{\prime}\right]\left[\frac{1}{p}\right] \\
& \mathrm{H}^{0}\left(\mathcal{J}_{\text {rig }, 1} / \mathrm{P}, \omega_{\text {rig }}^{\kappa}\right)
\end{aligned}
$$

D'après la proposition 5.3 on a un isomorphisme

$$
e_{\mathrm{P}} \mathrm{H}^{0}\left(\mathcal{T}_{\text {rig }, 1} / \mathrm{P}, \omega_{\text {rig }}^{\kappa}\right) \stackrel{\sim}{\rightarrow} e_{\mathrm{P}} \mathrm{H}^{0}\left(\mathcal{T}_{\text {rig }, \infty} / \mathrm{P}, \omega_{\text {rig }}^{\kappa}\right) .
$$

Il reste donc à montrer qu'on a un isomorphisme

$$
e_{\mathrm{P}} \mathrm{H}^{0}\left(\mathcal{J}_{\text {rig }, \infty} / \mathrm{P}, \omega_{\text {rig }}^{\kappa}\right) \stackrel{\sim}{\rightarrow} e_{\mathrm{P}} \mathrm{H}^{0}\left(\mathcal{J}_{\text {rig }, \infty} / \mathrm{P}, \Omega_{\mathrm{P}, \text { rig }}^{\kappa}\right)
$$

ou ce qui revient au même, un isomorphisme :

$$
e_{\mathrm{P}} \mathrm{H}^{0}\left(\mathcal{T}_{\infty} / \mathrm{P}, \tilde{\omega}^{\kappa}\right) \stackrel{\sim}{\rightarrow} e_{\mathrm{P}} \mathrm{H}^{0}\left(\mathcal{T}_{\infty} / \mathrm{P}, \Omega_{\mathrm{P}}^{\kappa}\right) \text {. }
$$

Pour tout $x \in \mathcal{J}_{\infty} / \mathrm{P}$ et tout faisceau cohérent $\mathcal{F}$ de $\theta_{\mathcal{T}_{\infty} / \mathrm{P}}$-modules, on note $\mathcal{F}_{x}=\operatorname{colim}_{x \in U} \mathcal{F}(U)$, où la limite inductive va sur les ouverts formels de $\mathcal{J}_{\infty} / \mathrm{P}$ qui contiennent $x$. Notons $\tilde{\omega}_{0}^{\kappa}$ le $\theta_{T_{\infty} / \mathrm{P}}$-module, noyau du morphisme res : $\tilde{\omega}^{\kappa} \rightarrow \Omega_{\mathrm{P}}^{\kappa}$. Considérons le diagramme commutatif suivant, dont les lignes sont le début des résolutions de Godement augmentées :

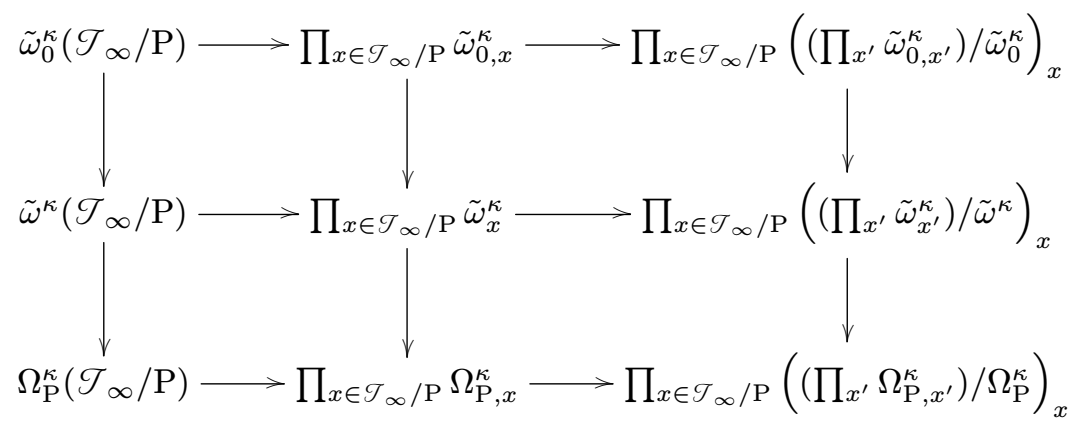

Les opérateurs $T_{\beta}^{1}$ agissent sur ce diagramme. La précaution consistant à prendre une résolution de Godement au niveau formel plutôt qu'au niveau 
rigide provient de la normalisation du morphisme trace du numéro 5.1.3. Cette normalisation est de nature locale pour le schéma formel $\mathcal{J}_{\infty} / \mathrm{P}$ mais pas pour l'espace rigide associé. Le morphisme

$$
\prod_{x \in \mathcal{T}_{\infty} / \mathrm{P}} \tilde{\omega}_{x}^{\kappa} \rightarrow \prod_{x \in \mathcal{T}_{\infty} / \mathrm{P}} \Omega_{\mathrm{P}, x}^{\kappa}
$$

est surjectif. Il résulte du lemme 5.1 et de la proposition 3.2 que

$$
e_{\mathrm{P}} \cdot \prod_{x \in \mathcal{T}} \tilde{\omega}_{\infty}^{\kappa} / \mathrm{P}=0
$$

d'où un isomorphisme :

$$
e_{\mathrm{P}} \cdot \prod_{x \in \mathcal{T}_{\infty} / \mathrm{P}} \tilde{\omega}_{x}^{\kappa} \stackrel{\sim}{\rightarrow} e_{\mathrm{P}} \cdot \prod_{x \in \mathcal{T}_{\infty} / \mathrm{P}} \Omega_{\mathrm{P}, x}^{\kappa} .
$$

Il résulte encore du lemme 5.1 et de la proposition 3.2 que

$$
e_{\mathrm{P}} \cdot \prod_{x \in \mathcal{G}}\left(\left(\prod_{x^{\prime} / \mathrm{P}} \tilde{\omega}_{0, x^{\prime}}^{\kappa}\right) / \tilde{\omega}_{0}^{\kappa}\right)_{x}=0 .
$$

Ceci montre bien l'isomorphisme

$$
e_{\mathrm{P}} \mathrm{H}^{0}\left(\mathcal{T}_{\infty} / \mathrm{P}, \tilde{\omega}^{\kappa}\right) \stackrel{\sim}{\rightarrow} e_{\mathrm{P}} \mathrm{H}^{0}\left(\mathcal{T}_{\infty} / \mathrm{P}, \Omega_{\mathrm{P}}^{\kappa}\right) .
$$

REMARQUE 5.4. - On a aussi, suivant la remarque 3.3, l'égalité $e_{\mathrm{P}} \mathrm{H}^{0}\left(\mathcal{J}_{\infty, 1} / \mathrm{P}, \omega^{\kappa}\right)=e_{\mathrm{P}} \mathrm{H}^{0}\left(\mathcal{T}_{\infty, 1} / \mathrm{P}, \tilde{\omega}^{\kappa}\right)$.

5.2.3. Le cas d'un poids Q-régulier. — On peut encore améliorer la « descente» de la proposition 5.4 lorsque le poids est Q-régulier et passer de $\mathcal{T}_{\infty, 1} / \mathrm{P}$ à $\phi_{\infty}$. Pour cela, il nous faut introduire un nouvel opérateur $\tilde{T}_{\beta_{\mathrm{P}}}$, suivant [7], p. 39-40.

Notons $\beta_{\mathrm{P}}=\operatorname{diag}\left(\alpha_{\mathrm{P}}, \nu\left(\beta_{\mathrm{P}}\right) \omega_{0} \alpha_{\mathrm{P}}^{-1} \omega_{0}\right)$. La double classe $K_{\mathrm{P}}(1) \beta_{\mathrm{P}} K_{\mathrm{S}}$ définit une correspondance que nous notons simplement $\tilde{\mathcal{T}}_{\infty, 1}^{\text {nc }} / \mathrm{P}$. Ses $R$-points sont la donnée de :

- Une variété abélienne ordinaire principalement polarisée $\mathscr{G} \stackrel{f}{\rightarrow} R$ et d'un P-drapeau : $\psi_{1}: \mu_{p}^{g} \simeq \mathscr{C}[p]^{0} \bmod \mathrm{P}$.

- Une variété abélienne ordinaire principalement polarisée $\mathscr{G}^{\prime} \stackrel{f^{\prime}}{\rightarrow} R$ et d'un P-drapeau : $\psi_{1}^{\prime}: \mu_{p}^{g} \simeq \mathscr{C}[p]^{0} \bmod \mathrm{P}$.

- Une $p$-isogénie de facteur de similitude $\nu\left(\beta_{\mathrm{P}}\right), \pi: \mathscr{G} \rightarrow \mathscr{C}^{\prime}$ telle qu'il existe (localement pour la topologie étale) des trivialisations $\psi_{\infty}: \mu_{p^{\infty}}^{g} \simeq$ $\mathscr{C}\left[p^{\infty}\right]^{0}$ et $\psi_{\infty}^{\prime}: \mu_{p^{\infty}}^{g} \simeq \mathscr{C}^{\prime}\left[p^{\infty}\right]^{0},\left.\psi_{\infty}^{\prime}\right|_{\mu_{p}^{g}}=\psi_{1}^{\prime} \bmod \mathrm{P}$ dans lesquelles

$$
\psi_{\infty}^{\prime-1} \circ \pi \circ \psi_{\infty}=\alpha_{\mathrm{P}}
$$


On dispose à nouveau de deux projections $p_{1}, p_{2}: \tilde{\mathcal{T}}_{\infty, 1}^{\mathrm{nc}} / \mathrm{P} \rightarrow \mathcal{T}_{\infty, 1}^{\mathrm{nc}} / \mathrm{P}$ et d'une immersion ouverte et fermée $\mathcal{T}_{\infty, 1}^{\mathrm{nc}} / \mathrm{P}\left(\beta_{\mathrm{P}}\right) \hookrightarrow \tilde{\mathcal{T}}_{\infty, 1}^{\mathrm{nc}} / \mathrm{P}$.

Soit $\bar{x}:$ Spec $k \rightarrow \tilde{\mathcal{J}}_{\infty, 1}^{\mathrm{nc}} / \mathrm{P}$ un point géométrique.

LEMme 5.2. - Il existe $w(\bar{x}) \in W / W_{\mathrm{M}}$ (peut-être pas unique) et $\psi^{\infty}: \mu_{p^{\infty}}^{g} \simeq$ $\mathscr{G}\left[p^{\infty}\right]^{0}, \psi_{\infty}=\psi_{1} \bmod \mathrm{P}, \psi_{\infty}^{\prime}: \mu_{p^{\infty}}^{g} \simeq \mathscr{G}\left[p^{\infty}\right]^{0}, \psi_{\infty}^{\prime}=\psi_{1}^{\prime} \bmod \mathrm{P}$ des trivialisations au dessus de Spec $k$ telles que

$$
\psi_{\infty}^{\prime-1} \circ \pi \circ \psi_{\infty}=\alpha_{\mathrm{P}} w(\bar{x}) .
$$

De plus $w(\bar{x})=1$ si et seulement si $\bar{x} \in \mathcal{J}_{\infty, 1}^{\mathrm{nc}} / \mathrm{P}\left(\beta_{\mathrm{P}}\right)$ et dans ce cas $w(\bar{x})$ est unique.

Démonstration. - Par définition de la correspondance $\tilde{\mathcal{J}}_{\infty, 1}^{\text {nc }} / \mathrm{P}$, il existe des trivialisations $\psi_{\infty}^{0}: \mu_{p^{\infty}}^{g} \simeq \mathscr{G}\left[p^{\infty}\right]^{0}$ et $\psi_{\infty}^{\prime}: \mu_{p^{\infty}}^{g} \simeq \mathscr{G}^{\prime}\left[p^{\infty}\right]^{0}, \psi_{\infty}^{\prime}=\psi_{1}^{\prime} \bmod \mathrm{P}$ dans lesquelles

$$
\psi_{\infty}^{\prime}-1 \circ \pi \circ \psi_{\infty}^{0}=\alpha_{\mathrm{P}}
$$

La trivialisation $\psi_{\infty}^{0} \bmod \mathrm{P}$ définit un P-drapeau dans $\mathscr{G}[p]^{0}$. Comme la position relative des $\mathrm{P}$-drapeaux est parametrée par $W_{\mathrm{P}}$, il existe $w \in W_{\mathrm{P}}$ tel que $\psi_{\infty}^{0} \circ w \bmod \mathrm{P}=\psi_{1}$. Posons donc $\psi_{\infty}=\psi_{\infty}^{0} \circ w$ et $w(\bar{x})=w^{-1}$. Si $w(\bar{x})=1$ alors $\bar{x} \rightarrow \mathcal{T}_{\infty, 1}^{\mathrm{nc}} / \mathrm{P}(\beta)$ par définition de la correspondance. Dans ce cas on vérifie bien que $w(\bar{x})$ est unique.

Soit $\kappa \in X\left(\mathrm{~T}_{\mathrm{Q}}\right)^{+}$. Nous définissons un morphisme $\tilde{\pi}^{\kappa}: p_{2}^{\star} \omega_{\text {rig }}^{\kappa} \rightarrow p_{1}^{\star} \omega_{\text {rig }}^{\kappa}$ par la règle :

$$
f \mapsto\left[\tilde{\pi}^{\kappa} f: \omega \mapsto \kappa^{\prime}\left(\alpha_{\mathrm{P}}\right) f\left(\omega^{\prime}\right)\right]
$$

où $\omega^{\prime}$ est tel que $\pi^{\star} \omega=\omega^{\prime}$.

Soit $\bar{x} \in \tilde{\mathcal{T}}_{\infty, 1} / \mathrm{P}\left(\overline{\mathbb{F}}_{p}\right)$. Notons $B$ l'hensélisé strict de $\mathcal{T}_{\infty, 1} / \mathrm{P}$ en $\bar{x}$. Notons $K=B[1 / p]$. Voici une généralisation du lemme 5.1 .

LEMME 5.3. - Il existe un diagramme commutatif où les applications verticales sont des isomorphismes

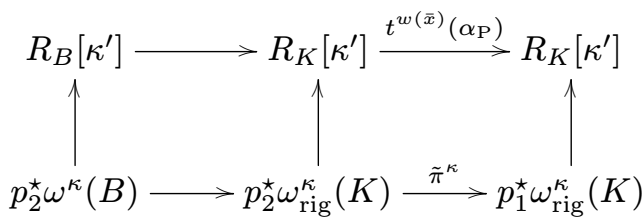

TOME $140-2012-\mathrm{N}^{\mathrm{O}} 3$ 
Démonstration. - D'après le lemme 5.2 , il existe $\psi^{\infty}: \mu_{p \infty}^{g} \simeq \mathscr{C}\left[p^{\infty}\right]^{0}, \psi_{\infty}=$ $\psi_{1} \bmod \mathrm{P}$ et $\psi_{\infty}^{\prime}: \mu_{p^{\infty}}^{g} \simeq \mathscr{C}\left[p^{\infty}\right]^{0}, \psi_{\infty}^{\prime}=\psi_{1}^{\prime} \bmod \mathrm{P}$ deux trivialisations au dessus de Spec $A$, compatibles avec les structures de niveau partielles et un élément $w=w(\bar{x}) \in W / W_{\mathrm{M}}$ tels que $\psi_{\infty}^{\prime-1} \circ \pi \circ \psi_{\infty}=\alpha_{\mathrm{P}} w$. Le diagramme suivant commute :

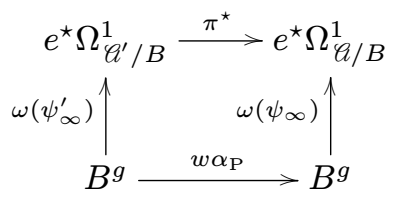

Il en résulte que $\tilde{\pi^{\kappa}} f\left(\omega\left(\psi_{\infty}\right) \circ g\right)=f\left(\omega\left(\psi_{\infty}^{\prime}\right) \circ \alpha_{\mathrm{P}}^{-1} w^{-1} \circ g \circ \alpha_{\mathrm{P}}\right)$.

Notons $\tilde{\operatorname{Tr}}=m_{\beta_{\mathrm{P}}}^{-1} \operatorname{Tr}_{p_{1}}:\left(p_{1}\right)_{\star} \theta_{\tilde{\mathcal{J}}_{\infty, 1}^{\text {nc }} / \mathrm{P}} \rightarrow \theta_{\mathcal{T}_{\infty, 1}^{\text {nc }} / \mathrm{P}}$ la trace normalisée. On dispose d'un opérateur

$$
\begin{aligned}
\tilde{T}_{\beta_{\mathrm{P}}}: \mathrm{H}^{0}\left(\mathcal{J}_{\text {rig, }, 1}^{\mathrm{nc}} / \mathrm{P}, \omega_{\text {rig }}^{\kappa}\right) \rightarrow & \mathrm{H}^{0}\left(\tilde{\mathcal{T}}_{\text {rig }, 1}^{\mathrm{nc}} / \mathrm{P}, p_{2}^{\star} \omega_{\text {rig }}^{\kappa}\right) \\
& \stackrel{\tilde{\pi}^{\kappa}}{\rightarrow} \mathrm{H}^{0}\left(\tilde{\mathcal{T}}_{\text {rig }, 1}^{\mathrm{nc}} / \mathrm{P}, p_{1}^{\star} \omega_{\text {rig }}^{\kappa}\right) \stackrel{\tilde{\mathrm{Tr}}}{\rightarrow} \mathrm{H}^{0}\left(\mathcal{T}_{\text {rig }, 1}^{\mathrm{nc}} / \mathrm{P}, \omega_{\text {rig }}^{\kappa}\right)
\end{aligned}
$$

Il résulte du principe de Koecher que $\tilde{T}_{\beta_{\mathrm{P}}} \in \operatorname{End}\left(\mathrm{H}^{0}\left(\mathcal{T}_{\text {rig, }, 1} / \mathrm{P}, \omega_{\text {rig }}^{\kappa}\right)\right)$ et cet opérateur respecte la cuspidalité.

Proposition 5.5. - Si $\kappa \in X\left(\mathrm{~T}_{\mathrm{Q}}\right)$ est Q-régulier, alors on a pour tout $f \in e_{\mathrm{P}} \mathrm{H}^{0}\left(\mathcal{J}_{\infty, 1} / \mathrm{P}, \omega^{\kappa}\right)$,

$$
T_{\beta_{\mathrm{P}}}^{1} \cdot f-\tilde{T}_{\beta_{\mathrm{P}}} \cdot f \in p \mathrm{H}^{0}\left(\mathcal{J}_{\infty, 1} / \mathrm{P}, \tilde{\omega}^{\kappa}\right)
$$

Démonstration. - On a $T_{\beta_{\mathrm{P}}}^{1} \cdot f-\tilde{T}_{\beta_{\mathrm{P}}} \cdot f=\left(T_{\beta_{\mathrm{P}}}^{1}-\tilde{T}_{\beta_{\mathrm{P}}}\right) \cdot f$. On peut donc appliquer les lemmes 5.2 et 5.3 pour se ramener au lemme 3.1 .

Comme l'image de $\tilde{T}_{\beta_{\mathrm{P}}}$ est constituée de sections $\mathrm{GL}_{g}\left(\mathbb{Z}_{p}\right)$-invariantes, l'opérateur $\tilde{T}_{\beta_{\mathrm{P}}}$ est à valeur dans $\mathrm{H}^{0}\left(S_{\infty}, \tilde{\omega}^{\kappa}\right)$. L'opérateur $T_{\beta_{\mathrm{P}}}^{1}$ est inversible sur le module $e_{\mathrm{P}}\left(\mathrm{H}^{0}\left(\mathcal{J}_{\infty, 1} / \mathrm{P}, \omega^{\kappa}\right)\right)$, on en déduit le corollaire suivant :

Corollaire 5.2. - Lorsque $\kappa$ est Q-régulier, l'inclusion naturelle $\mathrm{H}^{0}\left(\phi_{\infty}, \omega^{\kappa}\right) \rightarrow \mathrm{H}^{0}\left(\mathcal{J}_{\infty, 1} / \mathrm{P}, \omega^{\kappa}\right)$ induit un isomorphisme après la projection $e_{\mathrm{P}}$ :

$$
e_{\mathrm{P}} \mathrm{H}^{0}\left(\phi_{\infty}, \omega^{\kappa}\right) \stackrel{\sim}{\rightarrow} e_{\mathrm{P}} \mathrm{H}^{0}\left(\mathcal{T}_{\infty, 1} / \mathrm{P}, \omega^{\kappa}\right) .
$$

Démonstration. - Grâce à la proposition 5.5, on peut appliquer le lemme de Nakayama topologique à l'injection $e_{\mathrm{P}} \mathrm{H}^{0}\left(\phi_{\infty}, \omega^{\kappa}\right) \rightarrow e_{\mathrm{P}} \mathrm{H}^{0}\left(\mathcal{J}_{\infty, 1} / \mathrm{P}, \omega^{\kappa}\right)$. 
5.2.4. Le cas des formes avec un Nebentypus. - Soit $q$ un nombre premier ne divisant pas $N p, A$ l'anneau des entiers d'une extension fini de $\mathbb{Q}_{p}$, d'uniformisante $\pi$. Soit $\chi:(\mathbb{Z} / q \mathbb{Z})^{\times} \rightarrow A^{\times}$un caractère. Il est immédiat de généraliser la discussion précédente aux formes avec un Nebentypus. Le point est que les correspondances et les opérateurs définis en $p$ commutent avec l'action de $(\mathbb{Z} / q \mathbb{Z})^{\times}$. On obtient donc la proposition :

Proposition 5.6. - Soit $\mathrm{P}$ un parabolique standard de $\mathrm{GL}_{g}$ et $\kappa \in X\left(\mathrm{~T}_{\mathrm{Q}}\right)^{+}$ un caractère dominant. On a un isomorphisme

$$
e_{\mathrm{P}} \mathrm{H}^{0}\left(\left(\mathcal{J}_{0, q}\right)_{\infty, 1} / \mathrm{P}, \omega^{\kappa}(\chi)\right) \stackrel{\sim}{\rightarrow} e_{\mathrm{P}} V_{1, q, \infty}^{S \mathrm{P}}\left[\chi,-\kappa^{\prime}\right] .
$$

Si le poids est Q-régulier, on a de plus un isomorphisme

$$
e_{\mathrm{P}} \mathrm{H}^{0}\left(\left(\phi_{0, q}\right)_{\infty}, \omega^{\kappa}(\chi)\right) \simeq e_{\mathrm{P}} \mathrm{H}^{0}\left(\left(\mathcal{T}_{0, q}\right)_{\infty, 1} / \mathrm{P}, \omega^{\kappa}(\chi)\right)
$$

\section{Le théorème de relèvement et ses applications}

6.1. Le théorème de changement de base. - On insiste ici sur le fait que la structure de niveau $\mathcal{N}$ est telle que $\Gamma_{\mathcal{N}}$ est net. On dispose de morphismes de changement de base $\mathrm{H}^{0}\left(\phi, \omega^{\kappa}\right) \rightarrow \mathrm{H}^{0}\left(\phi_{m}, \omega^{\kappa}\right)$. Notons $\mathrm{H}_{\text {cusp }}^{0}\left(\phi_{m}, \omega^{\kappa}\right)$ le sous-module de $\mathrm{H}^{0}\left(\phi_{m}, \omega^{\kappa}\right)$ des formes qui s'annulent au bord.

THÉORÈme 6.1. - Si le poids $\kappa$ est parallèle, le morphisme de réduction modulo $p^{m}, \mathrm{H}^{0}\left(\&, \omega^{\kappa}\right) \rightarrow \mathrm{H}^{0}\left(\phi_{m}, \omega^{\kappa}\right)$ est surjectif.

De plus, il existe $N \in \mathbb{N}$, tel que pour tout poids parallèle $\kappa=\underline{k}$ avec $k \geq N$, les applications suivantes sont surjectives :

$$
\mathrm{M}\left(\kappa, \Gamma_{\mathscr{N}}, \mathbb{Z}_{p}\right) \rightarrow \mathrm{M}\left(\kappa, \Gamma_{\mathscr{N}}, \mathbb{F}_{p}\right) \text { et } \mathrm{S}\left(\kappa, \Gamma_{\mathscr{N}}, \mathbb{Z}_{p}\right) \rightarrow \mathrm{S}\left(\kappa, \Gamma_{\mathscr{N}}, \mathbb{F}_{p}\right)
$$

Démonstration. - Le faisceau inversible $\omega^{1}=\Lambda^{g} \Omega_{\mathscr{Q} / \bar{X}}^{1}$ descend à la compactification minimale, $X^{\star}$. On note encore $\omega^{1}$ le faisceau inversible sur la compactification minimale qui induit $\omega^{1}$ par image inverse. Il est ample sur la compactification minimale ([3], V, thm. 2.3). Il en résulte que l'image $\phi^{\star}$ de $\phi$ dans la compactification minimale est affine. Pour tout $\mathbb{Z}_{p}$-module $M$, et tout poids parallèle $\kappa=\underline{k}, \mathrm{H}^{0}\left(\phi, \omega^{\kappa} \otimes_{\mathbb{Z}_{p}} M\right)=\mathrm{H}^{0}\left(\phi^{\star}, \omega^{\kappa} \otimes_{\mathbb{Z}_{p}} M\right)$. Comme $\phi^{\star}$ est affine, il n'y a pas d'obstruction à relever les sections. De même, comme $\omega^{1}$ est ample, il existe $N$ tel que pour $k \geq N$ et $\kappa=\underline{k}, \mathrm{H}^{1}\left(X^{\star}, \omega^{\kappa} \otimes \mathbb{F}_{p}\right)=\mathrm{H}_{\text {cusp }}^{1}\left(X^{\star}, \omega^{\kappa} \otimes \mathbb{F}_{p}\right)=0$. Dans ce cas, il n'y a pas d'obstruction à relever les sections de la caractéristique $p$ vers la caractéristique 0 .

Dans le cas général, on a un théorème pour les formes cuspidales. Il est dû à Hida ([7], th. 3.1). La démonstration occupe la suite de cette section. 
ThÉORÈme 6.2. - Soit $\mathrm{P}$ un parabolique standard de $\mathrm{GL}_{g}, \mathrm{Q}=\omega_{0} \cdot{ }^{t} \mathrm{P} \cdot \omega_{0}$ et $\kappa \in X\left(\mathrm{~T}_{\mathrm{Q}}\right)$ un caractère. Les morphismes

$$
\mathrm{H}_{\text {cusp }}^{0}\left(\varnothing, \omega^{\kappa}\right) \rightarrow \mathrm{H}_{\text {cusp }}^{0}\left(\phi_{m}, \omega^{\kappa}\right) \quad \text { et } \quad V_{\infty, \text { cusp }}^{S P}\left[-\kappa^{\prime}\right] \rightarrow V_{m, \text { cusp }}^{S P}\left[-\kappa^{\prime}\right]
$$

sont surjectifs.

De plus, il existe $N(\kappa) \in \mathbb{N}$, tel que pour tout $k \geq N(\kappa)$, le morphisme

$$
\mathrm{S}\left(\kappa+\underline{k}, \Gamma_{\mathcal{N}}, \mathbb{Z}_{p}\right) \rightarrow \mathrm{S}\left(\kappa+\underline{k}, \Gamma_{\mathcal{N}}, \mathbb{F}_{p}\right)
$$

est surjectif.

On a des théorèmes analogues lorsque les formes ont un Nebentypus. Soit $q$ un nombre premier ne divisant pas $N p, A$ l'anneau des entiers d'une extension finie de $\mathbb{Q}_{p}$, d'uniformisante $\pi$ et $\chi:(\mathbb{Z} / q \mathbb{Z})^{\times} \rightarrow A^{\times}$une caractère.

ThÉORÈme 6.3. - Si le poids $\kappa$ est parallèle, le morphisme de réduction modulo $\pi^{m}$,

est surjectif.

$$
\mathrm{H}^{0}\left(\phi_{0, q}, \omega^{\kappa}(\chi)\right) \rightarrow \mathrm{H}^{0}\left(\left(\phi_{0, q}\right)_{m}, \omega^{\kappa}(\chi)\right)
$$

De plus, il existe $N \in \mathbb{N}$ tel que les morphismes suivant soient surjectifs si $\kappa=\underline{k}$ avec $k \geq N$ :

$$
\begin{aligned}
\mathrm{M}\left(\kappa, \Gamma_{\mathcal{N}}^{0}(q), \chi, A\right) & \rightarrow \mathrm{M}\left(\kappa, \Gamma_{\mathcal{N}}^{0}(q), \chi, A / \pi A\right) \\
\text { et } \mathrm{S}\left(\kappa, \Gamma_{\mathcal{N}}^{0}(q), \chi, A\right) & \rightarrow \mathrm{S}\left(\kappa, \Gamma_{\mathcal{N}}^{0}(q), \chi, A / \pi A\right)
\end{aligned}
$$

Dans le cas général, on a :

ThÉORÈme 6.4. - Soit $\mathrm{P}$ un parabolique standard de $\mathrm{GL}_{g}, \mathrm{Q}=\omega_{0} \cdot{ }^{t} \mathrm{P} \cdot \omega_{0}$ et $\kappa \in X\left(\mathrm{~T}_{\mathrm{Q}}\right)$ un caractère. Les morphisme de réduction modulo $\pi^{m}$ :

$$
\begin{aligned}
\mathrm{H}_{\text {cusp }}^{0}\left(\phi_{0, q}, \omega^{\kappa}(\chi)\right) & \rightarrow \mathrm{H}_{\text {cusp }}^{0}\left(\phi_{0, q}, \omega^{\kappa}(\chi) \otimes A / \pi^{m}\right) \\
\text { et } \quad V_{1, q, \infty, \text { cusp }}^{S \mathrm{P}}\left[\chi,-\kappa^{\prime}\right] & \rightarrow V_{1, q, m, \text { cusp }}^{S \mathrm{P}}\left[\chi,-\kappa^{\prime}\right]
\end{aligned}
$$

sont surjectifs.

De plus, il existe $N(\kappa) \in \mathbb{N}$, tel que pour tout $k \geq N(\kappa)$, le morphisme

$$
\mathrm{S}\left(\kappa+\underline{k}, \Gamma_{\mathcal{N}}^{0}(q), \chi, A\right) \rightarrow \mathrm{S}\left(\kappa+\underline{k}, \Gamma_{\mathcal{N}}^{0}(q), \chi, A / \pi A\right)
$$

est surjectif.

Nous allons démontrer le théorème 6.4. Les mêmes arguments démontrent le théorème 6.2. Nous reprenons le schéma de la démonstration de Hida dans [7]. Elle s'organise comme suit :

Soit $X_{0, q}^{\star}$ la compactification minimale de $X_{0, q}$ et $\pi: \bar{X}_{0, q} \rightarrow X_{0, q}^{\star}$ la projection vers la compactification minimale. On rappelle que $H \in \mathrm{H}^{0}\left(X_{0, q}^{\star}, \omega \underline{p-1} \otimes\right.$ $\mathbb{Z} / p \mathbb{Z}$ ) est l'invariant de Hasse. Grâce à la propriété d'amplitude du faisceau $\omega^{\underline{1}}$, 
il existe $t \in \mathbb{N}$ et un relèvement $E$ de $H^{t}$ à la caractéristique 0 . L'image de l'ouvert $\phi_{0, q}=\bar{X}_{0, q}[1 / E]$, de non annulation de $E$, dans $X_{0, q}^{\star}$ est un schéma affine par amplitude du faisceau $\omega^{t \underline{(p-1)}}$ sur la compactification minimale. Considérons les carrés cartésiens (le second est la restriction du premier au lieu de non annulation de $E$ ) :
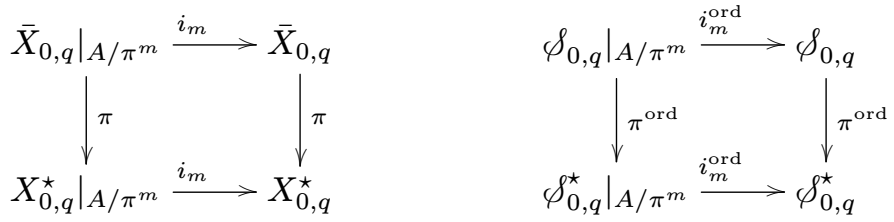

Considérons $\mathcal{T}_{0, q, \infty} / S \mathrm{P} \stackrel{f}{\rightarrow} \oint_{0, q, \infty}$ le quotient de la tour d'Igusa par le groupe $S \mathrm{P}\left(\mathbb{Z}_{p}\right)$. On note $\omega^{\text {top }, \mathrm{P}, \kappa}=f_{\star} \vartheta_{\mathcal{J}_{0, q, \infty} / S \mathrm{P}}\left[-\kappa^{\prime}\right]$ et $\omega_{\text {cusp }}^{\text {top }, \kappa}$ le sousfaisceau des sections qui s'annulent aux pointes.

Lemme 6.1. - Pour démontrer le théorème 6.4, il suffit donc de montrer que les morphismes de changement de base

$$
\begin{aligned}
i_{m}^{\star} \pi_{\star} \omega_{\text {cusp }}^{\kappa}(\chi) & \rightarrow \pi_{\star} i_{m}^{\star} \omega_{\text {cusp }}^{\kappa}(\chi) \\
\text { et } \quad\left(i_{m}^{\text {ord }}\right)^{\star} \pi_{\star}^{\text {ord }} \omega_{\text {cusp }}^{\text {top }, \kappa}(\chi) & \rightarrow \pi_{\star}^{\text {ord }}\left(i_{m}^{\text {ord }}\right)^{\star} \omega_{\text {cusp }}^{\text {top }, \text { P } \kappa}(\chi)
\end{aligned}
$$

sont des isomorphismes.

Démonstration. - En utilisant le fait que $\phi_{0, q}^{\star}$ est affine, on a

$$
\begin{aligned}
\mathrm{H}_{\text {cusp }}^{0}\left(\phi_{0, q}, \omega^{\kappa}(\chi)\right) \otimes A / \pi^{m} A & =\mathrm{H}_{\text {cusp }}^{0}\left(\phi_{0, q}^{\star}, \pi_{\star} \omega^{\kappa}(\chi)\right) \otimes A / \pi^{m} A \\
& =\mathrm{H}_{\text {cusp }}^{0}\left(\phi_{0, q}^{\star}, i_{m}^{\star} \pi_{\star} \omega^{\kappa}(\chi)\right) \\
& =\mathrm{H}_{\text {cusp }}^{0}\left(\phi_{0, q}^{\star}, \pi_{\star} i_{m}^{\star} \omega^{\kappa}(\chi)\right) \\
& =\mathrm{H}_{\text {cusp }}^{0}\left(\phi_{0, q}, i_{m}^{\star} \omega^{\kappa}(\chi)\right)
\end{aligned}
$$

on raisonne de même avec le faisceau $\omega_{\text {cusp }}^{\text {top, }, \kappa}(\chi)$.

Montrons le dernier point du théorème. Pour tout $k \in \mathbb{Z}$, on a la formule de projection :

$$
\pi_{\star} \omega_{\text {cusp }}^{\kappa+k}(\chi)=\omega^{\underline{k}} \otimes \pi_{\star} \omega_{\text {cusp }}^{\kappa}(\chi) .
$$

Comme $\omega \underline{1}$ est ample sur la compactification minimale, pour $k \gg 0$, on a l'annulation

$$
\mathrm{H}_{\text {cusp }}^{1}\left(X_{0, q}^{\star}, \pi_{\star} \omega^{\kappa+\underline{k}}(\chi)\right)=0 .
$$

On en déduit que $\mathrm{H}_{\text {cusp }}^{0}\left(X_{0, q}^{\star}, \pi_{\star} \omega^{\kappa+\underline{k}}(\chi)\right) \otimes A / \pi^{m} A=\mathrm{H}_{\text {cusp }}^{0}\left(X_{0, q}^{\star}, i_{m}^{\star} \pi_{\star} \omega^{\kappa+\underline{k}}(\chi)\right)$. On peut alors raisonner comme au dessus.

TOME $140-2012-\mathrm{N}^{\mathrm{O}} 3$ 
La démonstration des énoncés de changement de base du lemme est un calcul local pour la topologie $f p p f$. Ces morphismes de changement de base sont clairement des isomorphismes sur le complémentaire des pointes. Il s'agit donc de comprendre la projection $\pi$ au niveau des pointes. Pour ce faire, nous avons besoin de faire quelques rappels sur les compactifications toroïdales et minimales suivant [3] et [21].

6.1.0.1. Rappels sur les compactifications. - Commençons par introduire des données combinatoires. Soit $V=\oplus_{i=1}^{2 g} \mathbb{Z} e_{i}$ un $\mathbb{Z}$-module libre de rang $2 g$, muni d'une base $\left(e_{i}\right)_{i \in[1,2 g]}$. On le munit de la forme symplectique $<$, $>$ donné par la matrice $J$ dans cette base. Soit $\mathfrak{C}$ l'ensembles des sous-modules totalement isotropes facteurs directs de $V$. Pour tout $V^{\prime} \in \mathfrak{C}$, notons $C\left(V / V^{\prime} \perp\right)$ le cône des formes bilinéaires symétriques semi-définies positives sur $\left(V / V^{\prime} \perp\right) \otimes \mathbb{R}$, dont le radical est défini sur $\mathbb{Q}$. Pour tout $V^{\prime \prime} \subset V^{\prime}$, il existe une inclusion de $C\left(V / V^{\prime \prime} \perp\right)$ dans $C\left(V / V^{\prime} \perp\right)$. Notons $\mathscr{C}$ le quotient de l'union disjointe

$$
\coprod_{V^{\prime} \in \mathfrak{C}} C\left(V / V^{\prime}\right)
$$

par la relation d'équivalence engendrée par les inclusions de $C\left(V / V^{\prime \prime} \perp\right)$ dans $C\left(V / V^{\prime} \perp\right)$ pour tous facteurs directs totalement isotropes $V^{\prime \prime} \subset V^{\prime}$.

Soit l'entier $N^{\prime}=N q$. Considérons les groupes abéliens $\left(\mathbb{Z} / N^{\prime} \mathbb{Z}\right)^{2 g}$ et $(\mathbb{Z} / N \mathbb{Z})^{2 g}$ munis de la forme symplectique donnée par la matrice $J$ dans leurs bases canoniques. La base $\left(e_{i}\right)$ de $V^{\prime}$ nous fournit des isomorphisme symplectiques naturels $\psi_{N^{\prime}}: V / N^{\prime} V \rightarrow\left(\mathbb{Z} / N^{\prime} \mathbb{Z}\right)^{2 g}$ et $\psi_{N}: V / N V \rightarrow(\mathbb{Z} / N \mathbb{Z})^{2 g}$. Notons $\psi_{\mathcal{N}}$ la $\mathcal{N}$-orbite de $\psi_{N}$. Notons enfin $H$ le sous-groupe de $V / q V$ engendré par l'élément $Q$ qui est par définition la classe de $e_{1}$ dans $V / q V$.

Le groupe $\mathrm{G}(\mathbb{Z})$ agit sur $V$ et les stabilisateur de $\psi_{N^{\prime}}, \psi_{N}$ et $\psi_{\mathcal{N}}$ sont respectivement $\Gamma_{N^{\prime}}, \Gamma_{N}$ et $\Gamma_{\mathcal{N}}$.

Notons $\Gamma^{0}(q)$ et $\Gamma^{1}(q)$ les sous-groupes de $\mathrm{G}(\mathbb{Z})$ stabilisant $H$ et $Q$. Pour $i=0$ ou 1 notons enfin $\Gamma_{\mathcal{N}}^{i}(q)=\Gamma^{i}(q) \cap \Gamma_{\mathcal{N}}$. On a les inclusions de groupes

$$
\Gamma_{N^{\prime}} \subset \Gamma_{\mathcal{N}}^{1}(q) \subset \Gamma_{\mathcal{N}}^{0}(q)
$$

et le quotient $\Gamma_{\mathcal{N}}^{1}(q) \backslash \Gamma_{\mathcal{N}}^{0}(q)$ est isomorphe à $(\mathbb{Z} / q \mathbb{Z})^{\times}$. Notons $\mathcal{N}^{1}(q)$ et $\mathcal{N}^{0}(q)$ les images de $\Gamma_{\mathcal{N}}^{1}(q)$ et $\Gamma_{\mathcal{N}}^{0}(q)$ dans $\mathrm{G}\left(\mathbb{Z} / N^{\prime} \mathbb{Z}\right)$.

Soit $X_{N^{\prime}}$ l'espace de module des variétés abéliennes principalement polarisées de genre $g$, de niveau principal $N^{\prime}$. Le groupe $\mathrm{G}\left(\mathbb{Z} / N^{\prime} \mathbb{Z}\right)$ agit sur $X_{N^{\prime}}$ et on a des isomorphismes canoniques $X_{N^{\prime}} / \mathcal{N}^{1}(q) \simeq X_{1, q}$ et $X_{N^{\prime}} / \mathcal{N}^{0}(q) \simeq X_{0, q}$.

Choisissons une décomposition polyédrale rationnelle $\mathfrak{S}$ de $\mathscr{C}$ qui est $\Gamma_{N^{\prime}}$ admissible ([21], déf. 3.2.3.1). Il lui correspond une compactification toroïdale $\bar{X}_{N^{\prime}}$ ([3], IV th. 6.7). Le groupe $\mathrm{G}\left(N^{\prime}\right)$ agit sur cette compactification. Les quotients $\bar{X}_{N^{\prime}} / \mathcal{N}^{0}(q)$ et $\bar{X}_{N^{\prime}} / \mathcal{N}^{1}(q)$ sont des compactifications toroïdales $\bar{X}_{0, q}$ 
et $\bar{X}_{1, q}$ de $X_{0, q}$ et $X_{1, q}$. D'après [21], Chap. 5, le sous-groupe d'ordre $q$ universel $H$ au dessus de $X_{0, q}$ se prolonge en un sous-groupe fini et plat encore noté $H$ sur le schéma $\bar{X}_{0, q}([21]$, th 5.2.1). On a donc

$$
\bar{X}_{1, q}=\operatorname{Isom}_{\bar{X}_{0, q}}(\mathbb{Z} / q \mathbb{Z}, H) .
$$

On dispose d'une application $\mathfrak{S} \rightarrow \mathfrak{C}$ qui à toute face $\sigma$ de $\mathfrak{S}$ associe le plus petit facteur direct isotrope $V^{\prime} \in \mathfrak{C}$ tel que $\sigma \subset C\left(V / V^{\prime} \perp\right)$.

Sur $\bar{X}_{N^{\prime}}$ il y a une stratification fine paramétrée par $\mathfrak{S} / \Gamma_{N^{\prime}}$ au dessus d'une stratification grossière paramétrée par $\mathfrak{C} / \Gamma_{N^{\prime}}$ ([3] IV th. 6.7 et [21], prop. 3.3.1.2).

Soit $X_{N^{\prime}}^{\star}$ la compactification minimale de $X_{N^{\prime}}$. Et $\pi: \bar{X}_{N^{\prime}} \rightarrow X_{N^{\prime}}^{\star}$ la projection. Sur $X_{N^{\prime}}^{\star}$ on a une stratification paramétrée par $\mathfrak{C} / \Gamma_{N^{\prime}}$, compatible avec le morphisme $\pi$. Le groupe $\mathrm{G}\left(\mathbb{Z} / N^{\prime}\right)$ agit aussi sur la compactification minimale, de façon compatible avec le morphisme $\pi$. Les quotients $X_{N^{\prime}}^{\star} / \mathcal{N}_{q}^{0}$ et $X_{N^{\prime}}^{\star} / \mathcal{N}_{q}^{1}$ sont les compactifications minimales $X_{0, q}^{\star}$ et $X_{1, q}^{\star}$ de $X_{0, q}$ et $X_{1, q}$.

On a donc un diagramme commutatif, $\mathrm{G}\left(\mathbb{Z} / N^{\prime} \mathbb{Z}\right)$-équivariant

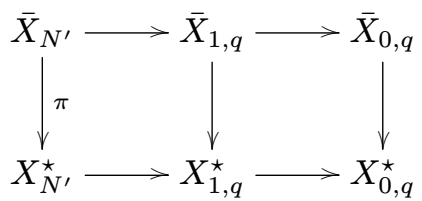

La présentation qui suit est empruntée au premier chapitre de [21]. Soit $V^{\prime}$ un élément de $\mathfrak{C}$. Soit $A_{V^{\prime}, N^{\prime}}$ l'espace de module des schémas abéliens $\mathscr{C}$ principalement polarisés, de dimension le rang de $V^{\prime}$, munis d'isomorphismes symplectiques $V^{\prime} \perp / V^{\prime} \otimes_{\mathbb{Z}} \mathbb{Z} / N^{\prime} \simeq \mathscr{G}\left[N^{\prime}\right]$. Soit $\mathscr{G}_{V^{\prime}, N^{\prime}} \rightarrow A_{V^{\prime}, N^{\prime}}$ le schéma abélien universel.

Posons $X_{V^{\prime}}=V / V^{\prime \perp}$ et $S_{V^{\prime}}=\operatorname{Sym}^{2} X_{V^{\prime}}$.

Soit $\mathcal{B}_{V^{\prime}, N^{\prime}}=\operatorname{Hom}_{A_{V^{\prime}, N^{\prime}}}\left(\frac{1}{N^{\prime}} X_{V^{\prime}}, \mathscr{G}_{V^{\prime}, N^{\prime}}\right)$. Au dessus de $\mathcal{B}_{V^{\prime}, N^{\prime}}$ on dispose d'une application universelle $c_{N^{\prime}}: \frac{1}{N^{\prime}} X_{V^{\prime}} \rightarrow \mathscr{G}_{V^{\prime}, N^{\prime}}$. Notons $c=\left.c_{N^{\prime}}\right|_{X_{V^{\prime}}}$. Soit $\mathrm{T}_{V^{\prime}}$ le tore de caractère $X_{V^{\prime}}$. $\mathrm{A} c$ on associe l'extension universelle

$$
0 \rightarrow \mathrm{T}_{V^{\prime}} \rightarrow \tilde{\mathscr{G}}_{V^{\prime}} \rightarrow \mathscr{C}_{V^{\prime}, N^{\prime}} \rightarrow 0 .
$$

On dispose ainsi d'un $\mathrm{GL}_{g}$-torseur

$$
\operatorname{Isom}\left(\theta_{\mathcal{B}_{V^{\prime}, N^{\prime}}}^{g}, e^{\star} \Omega^{1} \tilde{\mathscr{G}}_{V^{\prime}} / \mathscr{B}_{V^{\prime}, N^{\prime}}\right)
$$

et reprenant la construction du numéro 4.1 .1 on construit, pour tout poids $\kappa \in X(\mathrm{~T})$, des faisceaux cohérents $\omega^{\kappa}$ sur $\mathcal{B}_{V^{\prime}, N^{\prime}}$.

Notons $A_{V^{\prime}, N^{\prime}}^{\text {ord }}$ le lieu ordinaire de $A_{V^{\prime}, N^{\prime}}$ et $\mathscr{B}_{V^{\prime}, N^{\prime}}^{\text {ord }}=\mathcal{B}_{V^{\prime}, N^{\prime}} \times{ }_{A_{V^{\prime}, N^{\prime}}} A_{V^{\prime}, N^{\prime}}^{\text {ord }}$ On dispose d'une tour d'Igusa :

$$
\mathcal{J}_{\infty, V^{\prime}, N^{\prime}}=\operatorname{Isom}_{\mathcal{B}_{V^{\prime}, N^{\prime}}^{\text {ord }}}\left(\mu_{p^{\infty}}, \tilde{\mathscr{G}}_{V^{\prime}}^{\text {mult }}\left[p^{\infty}\right]\right) .
$$


Notons $f: \mathcal{T}_{\infty, V^{\prime}, N^{\prime}} / S \mathrm{P} \rightarrow \mathcal{B}_{V^{\prime}, N^{\prime}}^{\text {ord }}$ la tour d'Igusa quotienté par le groupe $S \mathrm{P}$. Pour tout parabolique standard $\mathrm{P}$ et tout caractère $\kappa \in X\left(\mathrm{~T}_{\mathrm{P}}\right)$, on peut donc considérer, au dessus de $\mathscr{B}_{V^{\prime}, N^{\prime}}^{\text {ord }}$, les faisceaux $\omega^{\text {top }, \mathrm{P}, \kappa}=$ $f_{\star} \vartheta_{\mathcal{T}_{\infty, V^{\prime}, N^{\prime}} / S \mathrm{P}}\left[\kappa^{\prime}\right]$ et le sous-faisceau des formes cuspidales $\omega_{\text {cusp }}^{\text {top, }, \kappa}$.

Notons $\mathscr{P} \rightarrow \mathscr{G}_{V^{\prime}, N^{\prime}} \times \mathscr{G}_{V^{\prime}, N^{\prime}}$ le fibré de Poincaré. Soit $\mathcal{M}_{V^{\prime}, N^{\prime}}$ le torseur sous $\operatorname{Hom}\left(\frac{1}{N^{\prime}} S_{V^{\prime}}, \mathbb{G}_{m}\right)$ des trivialisations symétriques de la biextension $\left(c_{N^{\prime}} \times\right.$ $\left.c_{N^{\prime}}\right)^{\star} \mathscr{P}^{N^{\prime}}$.

Le schéma $\mathcal{M}_{V^{\prime}, N^{\prime}}$ est l'espace de modules des 1-motifs principalement polarisés de rang $g$, rigidifiés par $X_{V^{\prime}}$ et munis d'une structure de niveau principale $M\left[N^{\prime}\right] \simeq V / N^{\prime} V$ induisant des isomorphismes $M^{\text {tor }}\left[N^{\prime}\right] \simeq V^{\prime} \otimes_{\mathbb{Z}} \mu_{N^{\prime}}$, $M^{a b}\left[N^{\prime}\right] \simeq V^{\prime} \perp / V^{\prime} \otimes_{\mathbb{Z}} \mathbb{Z} / N^{\prime}$ et $M^{e t}\left[N^{\prime}\right] \simeq V / V^{\prime} \perp \otimes_{\mathbb{Z}} \mathbb{Z} / N^{\prime}$.

Le stabilisateur dans $\mathrm{G}$ du facteur direct isotrope $V^{\prime}$ définit un sous-groupe parabolique que nous notons $\mathrm{P}_{V^{\prime}}$. Ce groupe est conjugué dans $\mathrm{G}(\mathbb{Z})$ au parabolique de $\mathrm{G}(\mathbb{Z})$ des matrices triangulaire par blocs de taille $r \times r, 2 g-r \times$ $2 g-r, r \times r\left(r\right.$ est le rang de $\left.V^{\prime}\right)$ :

$$
\left(\begin{array}{ccc}
A_{1} & C_{1} & D \\
0 & B & C_{2} \\
0 & 0 & A_{2}
\end{array}\right) .
$$

Soit $\mathrm{L}_{V^{\prime}}$ le sous-groupe de Levi de $\mathrm{P}_{V^{\prime}}$ des matrices conjuguées à

$$
\left(\begin{array}{ccc}
A_{1} & 0 & 0 \\
0 & B & 0 \\
0 & 0 & A_{2}
\end{array}\right)
$$

isomorphe à $\mathrm{GL}_{r} \times \mathrm{GSp}_{2 g-r} \times \mathrm{G}_{m}$. Soit $U_{V^{\prime}}$ le radical unipotent des matrices conjuguées à

$$
\left(\begin{array}{ccc}
1_{r} & C_{1} & D \\
0 & 1_{2 g-r} & C_{2} \\
0 & 0 & 1_{r}
\end{array}\right) .
$$

Le groupe $U_{V^{\prime}}$ est le produit semi-direct du groupe $\mathrm{C}_{V^{\prime}}$ des matrices conjuguées à

$$
\left(\begin{array}{ccc}
1_{r} & C_{1} & 0 \\
0 & 1_{2 g-r} & C_{2} \\
0 & 0 & 1_{r}
\end{array}\right)
$$


isomorphe au groupe $\operatorname{Hom}\left(\mathbb{Z}^{r}, \mathbb{Z}^{2 g-r}\right)$ par le groupe $\mathrm{D}_{V^{\prime}}$ des matrices conjuguées à

$$
\left(\begin{array}{ccc}
1_{r} & 0 & D \\
0 & 1_{2 g-r} & 0 \\
0 & 0 & 1_{r}
\end{array}\right)
$$

lui même isomorphe au groupe $\operatorname{Hom}\left(\operatorname{Sym}^{2} \mathbb{Z}^{r}, \mathbb{Z}\right)$.

Le groupe $\mathrm{P}_{V^{\prime}}\left(\mathbb{Z} / N^{\prime} \mathbb{Z}\right)$ agit sur $\mathcal{M}_{V^{\prime}, N^{\prime}}([3]$, IV 6.5). Cette action induit une action libre et transitive sur les structures de niveau principales $N^{\prime}$, compatibles avec $V^{\prime}$. De plus le dévissage précédent du groupe $P_{V^{\prime}}\left(\mathbb{Z} / N^{\prime} \mathbb{Z}\right)$ est compatible avec le dévissage $\mathcal{M}_{V^{\prime}, N^{\prime}} \rightarrow \mathcal{B}_{V^{\prime}, N^{\prime}} \rightarrow A_{V^{\prime}, N^{\prime}}$.

Posons $\Gamma_{\mathcal{N}, V^{\prime}}^{0}(q)=\mathrm{P}_{V^{\prime}}(\mathbb{Z}) \cap \Gamma_{\mathscr{N}}^{0}(q)$ et $\Gamma_{\mathcal{N}, V^{\prime}}^{1}(q)=\mathrm{P}_{V^{\prime}}(\mathbb{Z}) \cap \Gamma_{\mathcal{N}}^{1}(q)$.

Notons $\mathcal{N}_{V^{\prime}}^{0}(q)$ et $\mathcal{N}_{V^{\prime}}^{1}(q)$ leurs images respectives dans $\mathrm{P}_{V^{\prime}}\left(\mathbb{Z} / N^{\prime} \mathbb{Z}\right)$.

Les quotients $\mathcal{M}_{V^{\prime}, N^{\prime}} / \mathcal{N}_{V^{\prime}}^{0}(q)=\mathcal{M}_{V^{\prime}, \mathcal{N}, 0, q}$ et $\mathcal{M}_{V^{\prime}, N^{\prime}} / \mathcal{N}_{V^{\prime}}^{1}(q)=\mathcal{M}_{V^{\prime}, \mathcal{N}, 1, q}$ sont des schémas classifiant les 1-motifs $M$, principalement polarisés de genre $g$, rigidifiés par $X_{V^{\prime}}$, munis d'une structure de niveau $\mathcal{N}$ compatible avec $V^{\prime}$ et d'un sous-groupe d'ordre $q, H \subset M[q]$ (resp. d'un point d'ordre $q, Q \in M[q]$ ).

De façon plus précise, il existe un groupe abéliens libre

$$
S_{V^{\prime}} \subset S_{V^{\prime}, \mathcal{N}, 0, q} \subset \frac{1}{N^{\prime}} S_{V^{\prime}}
$$

et un dévissage

$$
\mathcal{M}_{V^{\prime}, \mathcal{N}, 0, q} \rightarrow \mathcal{B}_{V^{\prime}, \mathcal{N}, 0, q} \rightarrow A_{V^{\prime}, \mathcal{N}, 0, q}
$$

où :

- $A_{V^{\prime}, \mathcal{N}, 0, q}$ est l'espace de modules des schémas abéliens principalement polarisés de genre $g$, munis d'une structure de niveau induite par l'image de $\mathcal{N}_{V^{\prime}}^{0}(q)$ dans $\operatorname{GSp}\left(V^{\prime} \perp / V\right)\left(\mathbb{Z} / N^{\prime} \mathbb{Z}\right)$.

- $\mathscr{B}_{V^{\prime}, \mathcal{N}, 0, q}$ est un schéma abélien sur $A_{V^{\prime}, \mathcal{N}, 0, q}$ isogène au produit de $r$ copies du schéma abélien universel $\mathscr{G}_{V^{\prime}, \mathcal{N}, 0, q}$.

- $\mathcal{M}_{V^{\prime}, \mathcal{N}, 0, q} \rightarrow \mathcal{B}_{V^{\prime}, \mathcal{N}, 0, q}$ est un torseur sous le tore $\operatorname{Hom}\left(S_{V^{\prime}, \mathcal{N}, 0, q}, \mathbb{G}_{m}\right)$.

De plus

$$
\mathcal{M}_{V^{\prime}, \mathcal{N}, 1, q}=\operatorname{Isom}_{\mathcal{M}_{V^{\prime}, \mathcal{N}, 0, q}}(\mathbb{Z} / q \mathbb{Z}, H) .
$$

Le schéma $\mathcal{M}_{V^{\prime}, \mathcal{N}, 0, q}$ est affine au dessus de $\mathcal{B}_{V^{\prime}, \mathcal{N}, 0, q}$ et est le spectre d'un faisceau d' $\theta_{\mathcal{B}_{V^{\prime}, \mathfrak{x}, 0, q}}$-algèbre cohérente $\mathcal{L}$. Cette algèbre se décompose sous l'action du tore $\operatorname{Hom}\left(S_{V^{\prime}, \mathcal{N}, 0, q}, \mathbb{G}_{m}\right)$ :

$$
\mathscr{L}=\bigoplus_{\lambda \in S_{V^{\prime}, \mathscr{V}, 0, q}} \mathscr{L}(\lambda) .
$$

Soit $k$ un corps séparablement clos et $\bar{x}: \operatorname{Spec} k \rightarrow X_{0, q}^{\star}$ un point géométrique reposant dans la $V^{\prime}$-strate. La donnée de $\bar{x}$ est équivalente à la donnée

TOME $140-2012-\mathrm{N}^{\mathrm{O}} 3$ 
d'un point géométrique (noté de la même façon) $\bar{x}:$ Spec $k \rightarrow A_{V^{\prime}, \mathcal{N}^{\prime}, 0, q}$. Notons $\theta_{X_{0, q}^{\star}, \bar{x}}$ le localisé strict de $X_{0, q}^{\star}$ en $\bar{x}$ et $\hat{\theta}_{X_{0, q}^{\star}, \bar{x}}$ son complété au point fermé.

Soit $A_{V^{\prime}, \mathcal{N}^{\prime}, 0, q, \bar{x}}$ le localisé strict de $A_{V^{\prime}, \mathcal{N}, 0, q}$ en $\bar{x}$. Notons $\hat{\mathcal{B}}_{V^{\prime}, \mathcal{N}, 0, q, \bar{x}}$ le complété formel de $\mathscr{B}_{V^{\prime}, \mathcal{N}, 0, q} \times A_{V^{\prime}, \mathcal{N}, 0, q, \bar{x}}$ le long de la fibre de $\mathscr{B}_{V^{\prime}, \mathcal{N}, 0, q} \rightarrow$ $A_{V^{\prime}, \mathcal{N}, 0, q}$ en $\bar{x}$. La proposition qui suit est essentiellement contenue dans [3], V th. 2.7. On peut aussi consulter [21], prop. 4.2.6.

Proposition 6.1. - On a un isomorphisme canonique :

$$
\hat{\vartheta}_{X_{0, q}^{\star}, \bar{x}} \stackrel{\sim}{\longrightarrow}\left(\prod_{\lambda \in S_{V^{\prime}, \mathcal{N}, 0, q} \cap C\left(V / V^{\prime} \perp\right)^{\vee}} \mathrm{H}^{0}\left(\hat{\mathcal{B}}_{V^{\prime}, \mathcal{N}, 0, q, \bar{x}}, \mathscr{L}(\lambda)\right)\right)^{\Gamma_{\mathscr{N}, V^{\prime}}^{0}(q)}
$$

et pour tout caractère $\chi: \mathbb{Z} / q \mathbb{Z}^{\times} \rightarrow A^{\times}$, on a (non canoniquement si $\chi \neq 1$ ) : $\mathrm{H}^{0}\left(\operatorname{Spec} \hat{\theta}_{X_{0, q}^{\star}, \bar{x}}, \pi_{\star} \omega^{\kappa}(\chi)\right) \stackrel{\sim}{\longrightarrow}\left(\prod_{\lambda \in S_{V^{\prime}, \mathcal{N}, 0, q} \cap C\left(V / V^{\prime} \perp\right)^{\vee}} \mathrm{H}^{0}\left(\hat{\mathcal{B}}_{V^{\prime}, \mathcal{N}, 0, q, \bar{x}}, \mathscr{L}(\lambda) \otimes \omega^{\kappa}\right)\right)^{\Gamma_{\mathscr{N}, V^{\prime}}^{0}(q)}$.

Si $k$ est de caractéristique $p$ et $\bar{x} \in \phi_{0, q}^{\star}(k)$, on a de même

$\mathrm{H}^{0}\left(\operatorname{Spec} \hat{\theta}_{X_{0, q}^{\star}, \bar{x}}, \pi_{\star}^{\text {ord }} \omega^{\text {top }, \mathrm{P}, \kappa}(\chi)\right) \stackrel{\sim}{\longrightarrow}\left(\prod_{\lambda} \mathrm{H}^{0}\left(\hat{\mathcal{B}}_{V^{\prime}, \mathcal{N}, 0, q, \bar{x}}, \mathcal{L}(\lambda) \otimes \omega^{\text {top }, \mathrm{P}, \kappa}\right)\right)^{\Gamma_{\mathscr{N}, V^{\prime}}^{0}(q)}$ avec $\lambda \in S_{V^{\prime}, \mathcal{N}, 0, q} \cap C\left(V / V^{\prime} \perp\right)^{\vee}$.

Démonstration. - La décomposition polyédrale $\mathfrak{S}$ de $\mathscr{C}$ induit un plongement torique

$$
\mathcal{M}_{V^{\prime}, \mathcal{N}, 0, q} \hookrightarrow \overline{\mathcal{M}}_{V^{\prime}, \mathcal{N}, 0, q} .
$$

Soit $\hat{\bar{M}}_{V^{\prime}, \mathcal{N}, 0, q}$ la complétion le long de la strate fermé. Par construction on a

$$
\hat{\bar{M}}_{V^{\prime}, \mathcal{N}, 0, q} / \Gamma_{\mathcal{N}, V^{\prime}}^{0}(q) \simeq \hat{\bar{X}}_{0, q}^{V^{\prime}}
$$

où $\hat{\bar{X}}_{0, q}^{V^{\prime}}$ est la complétion de $\bar{X}_{0, q}$ le long de la $V^{\prime}$-strate. Notons $\hat{\bar{X}}_{0, q, \bar{x}}^{V^{\prime}}$ la complétion de $\hat{\bar{X}}_{0, q}^{V^{\prime}}$ le long de la fibre du morphisme $\bar{X}_{0, q} \rightarrow X_{0, q}^{\star}$. D'après [5] III 4.1.5, on a $\hat{\vartheta}_{X_{0, q}^{\star}, \bar{x}}=\mathrm{H}^{0}\left(\hat{\bar{X}}_{0, q, \bar{x}}^{V^{\prime}}, \theta_{\bar{X}_{0, q}}\right)$ et le premier isomorphisme découle de la description des fonctions régulières sur $\hat{\bar{M}}_{V^{\prime}, \mathcal{N}, 0, q}$. Les isomorphismes restant pour le caractère $\chi=1$ sont une version avec coefficient du premier isomorphisme. Ils se démontrent de la même façon.

Le revêtement étale $\bar{X}_{1, q} \rightarrow \bar{X}_{0, q}$ est trivial au dessus de $\hat{\bar{X}}_{0, q, \bar{x}}^{V^{\prime}}$ car le groupe $H$ est constant. En effet, il y a trois cas à considérer selon la position de $H$ par rapport à la filtration à trois crans sur le 1-motif ([21], 1.2.4). Si $H$ est torique, son groupe de cocaractère est constant, donné par le groupe $H \subset$ $V / q V \cap V^{\prime} / q V^{\prime}$. Il en résulte que $\operatorname{Isom}(\mathbb{Z} / q \mathbb{Z}, H)$ est un groupe constant (car la 
base contient les racines $q$-ièmes de l'unité). Si $H$ est abélien, $H$ est donné au dessus de $A_{V^{\prime}, \mathcal{N}^{\prime}, 0, q, \bar{x}}$ dans la $q$-torsion constante du schéma abélien universel. Si $H$ est étale, il est donné par l'image (non triviale) de $H \subset V / q V$ dans $V / V^{\prime} \perp$, qui est constante.

Il en résulte que les sections $\chi$-variantes de $\omega^{\kappa}$ au dessus de $\bar{X}_{1, q} \times_{\bar{X}_{0, q}} \hat{\bar{X}}_{0, q, \bar{x}}^{V^{\prime}}$ s'identifient (non-canoniquement) aux section de $\omega^{\kappa}$ au dessus de $\hat{\bar{X}}_{0, q, \bar{x}}^{V^{\prime}}$. On raisonne de même avec les sections de $\omega^{\text {top,P, }, \kappa}(\chi)$ ou $\tilde{\omega}^{\kappa}(\chi)$. Ceci permet bien de conclure.

Lemme 6.2. - Pour tout $\lambda \in S_{V^{\prime}, \mathcal{N}, 0, q} \cap C\left(V / V^{\prime} \perp\right)^{\vee}$ défini positif, la formation de $\mathrm{H}^{0}\left(\hat{\mathcal{B}}_{V^{\prime}, \mathcal{N}, 0, q, \bar{x}}, \mathcal{L}(\lambda) \otimes \omega^{\kappa}\right)$ commute au changement de base. Lorsque $\bar{x} \in S_{0, q}^{\star}(k)$ avec $k$ de caractéristique $p$, la formation de $\mathrm{H}^{0}\left(\hat{\mathcal{B}}_{V^{\prime}, \mathcal{N}, 0, q, \bar{x}}, \mathscr{L}(\lambda) \otimes\right.$ $\left.\omega^{\text {top, } \mathrm{P}, \kappa}\right)$ commute aussi au changement de base.

Démonstration. - Au dessus de $\mathscr{B}_{V^{\prime}, \mathcal{N}, 0, q}$, le faisceau localement libre $e^{\star} \Omega_{\tilde{\mathscr{G}}_{V^{\prime}} / \mathscr{B}_{V^{\prime}, \mathcal{N}, 0, q}}^{1}$ est une extension

$$
0 \rightarrow e^{\star} \Omega_{\mathscr{G}_{V^{\prime}} / \mathscr{B}_{V^{\prime}, \mathscr{N}, 0, q}}^{1} \rightarrow e^{\star} \Omega_{\tilde{\mathscr{G}}_{V^{\prime}} / \mathscr{B}_{V^{\prime}, \mathscr{N}, 0, q}}^{1} \rightarrow e^{\star} \Omega_{\mathrm{T}_{V^{\prime}} / \mathscr{B}_{V^{\prime}, \mathfrak{N}, 0, q}}^{1} \rightarrow 0 .
$$

Au dessus de $\hat{\mathcal{B}}_{V^{\prime}, \mathcal{N}, 0, q, \bar{x}}$, les deux faisceaux aux extrémités de la suite exacte sont libres. Il en résulte que pour tout poids $\kappa$, le faisceau $\omega^{\kappa}$ est une extension de faisceaux libres. Rappelons que $\mathcal{B}_{V^{\prime}, \mathcal{N}, 0, q}$ est un schéma abélien, et que le faisceau $\mathscr{L}(\lambda)$ est ample. Le premier point résulte donc du théorème d'annulation de Mumford [15], III, 16.

$\mathrm{Au}$ dessus de $\mathcal{B}_{V^{\prime}, \mathcal{N}, 0, q}^{\text {ord }}$, on a la tour d'Igusa :

$$
\mathcal{T}_{\infty, V^{\prime}, \mathcal{N}, 0, q}=\operatorname{Isom}_{\mathscr{B}_{V^{\prime}, \mathcal{N}, 0, q}^{\text {ord }}}\left(\mu_{p^{\infty}}, \tilde{\mathscr{G}}_{V^{\prime}}^{\text {mult }}\left[p^{\infty}\right]\right)
$$

Notons $\mathcal{B}_{m, V^{\prime}, \mathcal{N}, 0, q}^{\text {ord }}$ la réduction modulo $p^{m}$ de $\mathscr{B}_{V^{\prime}, \mathcal{N}, 0, q}^{\text {ord }}$ et

$$
f_{m, n}: \mathcal{T}_{m, n, V^{\prime}, \mathcal{N}, 0, q} / \mathrm{P} \rightarrow \mathcal{B}_{m, V^{\prime}, \mathcal{N}, 0, q}^{\text {ord }}
$$

la réduction modulo $p^{m}$ de la tour d'Igusa tronquée au cran $n$ et quotientée par P. Le morphisme $f_{m, n}$ est fini et étale, il résulte d'un théorème de Lang et Serre (voir [15] IV, 18) que chaque composante connexe de $\mathcal{J}_{m, n, V^{\prime}, \mathcal{N}, 0, q} / \mathrm{P}$ est un schéma abélien et que la restriction de $f_{m, n}$ à chaque composante connexe est une isogénie. On en déduit que le faisceau $f_{m, n}^{\star} \mathscr{L}(\lambda)$ est ample, et par le théorème d'annulation [15], III, 16, que la formation de $\mathrm{H}^{0}\left(\hat{\mathcal{J}}_{m, n, V^{\prime}, \mathcal{N}, 0, q, \bar{x}} / \mathrm{P}, \mathscr{O} \otimes\right.$ $f_{m, n}^{\star} \mathcal{L}(\Lambda)$ ) commute au changement de base (on a noté $\mathscr{O}$ le faisceau structural). Voilà qui démontre le second point lorsque le caractère $\kappa$ est trivial. Pour $\kappa$ général, on reprend l'argument précédent en remplaçant le faisceau structural $\mathscr{O}$ par le faisceau inversible $\mathscr{O}(\kappa)$ des fonctions régulières $-\kappa^{\prime}$-variantes 
sur $\mathcal{J}_{m, n, V^{\prime}, \mathcal{N}, 0, q} / S$ P. Comme le faisceau $\mathscr{O}(\kappa)$ est algébriquement équivalent à zéro, le théorème d'annulation s'applique.

Soit $\bar{x}:$ Spec $k \rightarrow X_{0, q}^{\star}$ un point géométrique de la fibre spéciale de $X_{0, q}^{\star}$.

Proposition 6.2. - Le morphisme de changement de base

$$
\mathrm{H}^{0}\left(\operatorname{Spec} \hat{\theta}_{X_{0, q}^{\star}, \bar{x}}, \pi_{\star} \omega_{\text {cusp }}^{\kappa}(\chi)\right) \rightarrow \mathrm{H}^{0}\left(\operatorname{Spec} \hat{\theta}_{X_{0, q}^{\star}, \bar{x}} \otimes A / \pi A, \pi_{\star} \omega_{\text {cusp }}^{\kappa}(\chi)\right)
$$

est surjectif.

De même si $\bar{x} \in S_{0, q}^{\star}(k)$, le morphisme de changement de base $\mathrm{H}^{0}\left(\operatorname{Spec} \hat{\theta}_{X_{0, q}^{\star}, \bar{x}}, \pi_{\star}^{\text {ord }} \omega_{\text {cusp }}^{\text {top }, \kappa}(\chi)\right) \rightarrow \mathrm{H}^{0}\left(\operatorname{Spec} \hat{\theta}_{X_{0, q}^{\star}, \bar{x}} \otimes A / \pi A, \pi_{\star}^{\text {ord }} \omega_{\text {cusp }}^{\text {top }, \mathrm{P}, \kappa}(\chi)\right)$ est surjectif.

Démonstration. - Le groupe $\Gamma_{V^{\prime}, \mathcal{N}, 0, q}$ agit sur $V^{\prime}$ à travers un sous groupe d'indice fini de $\operatorname{GL}\left(V^{\prime}\right)$, noté $\mathrm{GL}\left(V^{\prime}\right)_{\mathcal{N}, 0, q}$. Il agit donc sur $S_{V^{\prime}, \mathcal{N}, 0, q}$ à travers ce groupe. Le stabilisateur d'un élément $\lambda \in S_{V^{\prime}, \mathcal{N}, 0, q}$ positif et non dégénéré sous l'action de $\mathrm{GL}\left(V^{\prime}\right)_{\mathcal{N}, 0, q}$ est un groupe fini et donc trivial par netteté. Pour les faisceaux des formes cuspidales, les formules de la proposition 6.1 ne font intervenir effectivement que les éléments $\lambda \in S_{V^{\prime}, \mathcal{N}, 0, q}$ qui sont définis positifs. Comme le groupe $\mathrm{GL}\left(V^{\prime}\right)_{\mathcal{N}, 0, q}$ agit librement sur ces éléments, on est ramené au lemme 6.2.

6.2. Densité des formes classiques. - Soit $\mathrm{P}$ un parabolique standard de $\mathrm{GL}_{g}$, $\mathrm{Q}=\omega_{0} \cdot{ }^{t} \mathrm{P} . \omega_{0}$ et $\kappa \in X\left(\mathrm{~T}_{\mathrm{Q}}\right)$. On a définit au numéro 4.2.1 un morphisme de comparaison $\Theta_{\mathrm{P}}^{\star}: \mathrm{S}\left(\kappa, \Gamma_{\mathcal{N}}, \mathbb{Z}_{p}\right) \rightarrow V_{\text {cusp }, \infty}^{S \mathrm{P}}$. Le théorème de changement de base précédent a le corollaire important dû à Hida lorsque $\mathrm{P}=\mathrm{B}$ ([7], cor. 3.4). La démonstration s'adapte sans problème à notre cas plus général :

Corollaire 6.1 (Densité des formes classiques). - Pour tout parabolique $\mathrm{P}$, le module

$$
\left(\bigoplus_{\kappa \in X\left(\mathrm{~T}_{\mathrm{Q}}\right)} \Theta_{\mathrm{P}}^{\star}\left(\mathrm{S}\left(\kappa, \Gamma_{\mathcal{N}}, \mathbb{Z}_{p}\right)\right) \otimes \mathbb{Q}_{p}\right) \cap V_{\mathrm{cusp}, \infty}^{S \mathrm{P}}
$$

est dense dans $V_{\mathrm{cusp}, \infty}^{S \mathrm{P}}$.

Pour $\mathrm{P}=\mathrm{GL}_{g}$ on a le même énoncé sans supposer les formes cuspidales.

Un théorème analogue vaut pour les formes possédant un Nebentypus. Soit $q$ un nombre premier, premier à $N p$ et $A$ l'anneau des entiers d'une extension finie de $\mathbb{Q}_{p}$ d'uniformisante $\pi$. Soit $\chi:(\mathbb{Z} / q \mathbb{Z})^{\times} \rightarrow A^{\times}$un caractère. 
ThÉORÈme 6.5. - Pour tout parabolique $\mathrm{P}$ et $\mathrm{Q}=\omega_{0} \cdot{ }^{t} \mathrm{P} \cdot \omega_{0}$, le module

$$
\left(\bigoplus_{\kappa \in X\left(\mathrm{~T}_{\mathrm{P}}\right)} \Theta_{\mathrm{P}}^{\star}\left(\mathrm{S}\left(\kappa, \Gamma_{\mathcal{N}}^{0}(q), \chi, A\right)\right) \otimes \mathbb{Q}_{p}\right) \cap V_{1, q, \text { cusp }, \infty}^{S \mathrm{P}}[\chi]
$$

est dense dans $V_{1, q, \mathrm{cusp}, \infty}^{S \mathrm{P}}[\chi]$.

Pour $\mathrm{P}=\mathrm{GL}_{g}$ on a le même énoncé sans supposer les formes cuspidales.

6.3. Finitude et indépendance du rang. — Le théorème qui suit est une généralisation d'un résultat de Jochnowitz ([10]). Sa démonstration utilise les résultats de l'appendice. Il est possible de donner une démonstration via l'application d'Eichler-Shimura pour se ramener à l'énoncé correspondant en cohomologie de Betti ([8], 4.2.2).

ThÉORÈme 6.6. - Soit $\mathrm{P}$ un parabolique standard de $\mathrm{GL}_{g}, \mathrm{Q}=\omega_{0} \cdot{ }^{t} \mathrm{P} . \omega_{0}$. Pour tout poids $\kappa \in X\left(\mathrm{~T}_{\mathrm{Q}}\right)$, le module $e_{\mathrm{P}} V_{\text {cusp }, \infty}^{S \mathrm{P}}\left[-\kappa^{\prime}\right]$ est libre de rang fini. Ce rang est indépendant de la classe de $\kappa$ modulo $p-1$.

De plus, lorsque $\kappa$ est Q-très régulier, $e_{\mathrm{P}} \mathrm{S}\left(\kappa, \Gamma_{\mathcal{N}}, \mathbb{Z}_{p}\right)=e_{\mathrm{P}} V_{\text {cusp }, \infty}^{S \mathrm{P}}\left[-\kappa^{\prime}\right]$.

Le même énoncé vaut sans supposer la cuspidalité si $\mathrm{P}=\mathrm{GL}_{g}$.

Démonstration. - Le second point se déduit du théorème A.1 de l'appendice et du corollaire 5.2 (Noter que le projecteur $e$ de l'appendice associé à l'opérateur $U_{p}=T_{\beta_{0}}^{1}$ est contenu dans le projecteur $e_{\mathrm{P}}$ ). Voici comment déduire le premier point. D'une part, $e_{\mathrm{P}} V_{\text {cusp }, \infty}\left[-\kappa^{\prime}\right] \otimes_{\mathbb{Z}_{p}} \mathbb{F}_{p}=e_{\mathrm{P}} V_{\text {cusp }, 1}^{S \mathrm{P}}\left[-\kappa^{\prime}\right]$, d'après le théorème 6.2. Comme $e_{\mathrm{P}} V_{\mathrm{cusp}, 1}^{S \mathrm{P}}\left[-\kappa^{\prime}\right]$ ne dépend que de la classe de $\kappa$ modulo $p-1$, on peut supposer que $\kappa$ est Q-très régulier. Dans ce cas, $e_{\mathrm{P}} \mathrm{S}\left(\kappa, \Gamma_{\mathcal{N}}, \mathbb{Z}_{p}\right)=$ $e_{\mathrm{P}} V_{\mathrm{cusp}, \infty}^{S \mathrm{P}}\left[-\kappa^{\prime}\right]$ est bien de dimension finie.

On a les mêmes résultats lorsque les formes ont des Nebentypus. Soit $q$ un nombre premier ne divisant pas $N p$ et $A$ l'anneau des entiers d'une extension finie de $\mathbb{Q}_{p}$, d'uniformisante $\pi$. Soit $\chi:(\mathbb{Z} / q \mathbb{Z})^{\times} \rightarrow A^{\times}$un caractère.

THÉORÈme 6.7. - Soit $\mathrm{P}$ un parabolique standard de $\mathrm{GL}_{g}, \mathrm{Q}=\omega_{0} \cdot{ }^{t} \mathrm{P} . \omega_{0}$. Pour tout poids $\kappa \in X\left(\mathrm{~T}_{\mathrm{Q}}\right)$, le module $e_{\mathrm{P}} V_{\text {cusp }, \infty}\left[\chi,-\kappa^{\prime}\right]$ est libre de rang fini. Ce rang est indépendant de la classe de $\kappa$ modulo $p-1$.

De plus, lorsque $\kappa$ est Q-très régulier $e_{\mathrm{P}} \mathrm{S}\left(\kappa, \Gamma_{\mathcal{N}}^{0}(q), \chi, A\right)=e_{\mathrm{P}} V_{1, q, \text { cusp }, \infty}^{S \mathrm{P}}\left[\chi,-\kappa^{\prime}\right]$.

Le même énoncé vaut sans supposer la cuspidalité si $\mathrm{P}=\mathrm{GL}_{g}$.

TOME $140-2012-\mathrm{N}^{\circ} 3$ 


\section{Le théorème de contrôle vertical}

Soit $\mathrm{P}$ un parabolique standard de $\mathrm{GL}_{g}$. Notons $\mathrm{Q}=\omega_{0} \cdot{ }^{t} \mathrm{P} . \omega_{0}$. Soit $\mathcal{V}^{\text {ord- } \mathrm{P}}=$ $\operatorname{colim}_{m \geq 0} e_{\mathrm{P}} V_{m}^{S \mathrm{P}}$ et $\mathcal{V}_{\text {cusp }}^{\text {ord-P }}$ le sous-module des formes cuspidales. Soit $\mathcal{V}^{\text {ord-P, },}$ et $\mathcal{V}_{\text {cusp }}^{\text {ord-P, }}$ les duaux à valeur dans $\mathbb{Q}_{p} / \mathbb{Z}_{p}$ de $\mathcal{V}^{\text {ord-P }}$ et $\mathcal{V}_{\text {cusp }}^{\text {ord-P }}$.

Soit $\Gamma_{\mathrm{Q}}$ le pro-p sous-groupe maximal de $\mathrm{T}_{\mathrm{Q}}\left(\mathbb{Z}_{p}\right)$ donné par la suite exacte $1 \rightarrow \Gamma_{\mathrm{Q}} \rightarrow \mathrm{T}_{\mathrm{Q}}\left(\mathbb{Z}_{p}\right) \rightarrow \mathrm{T}_{\mathrm{Q}}(\mathbb{Z} / p) \rightarrow 1$. Le relèvement de Teichmüller scinde la suite précédente et on a un isomorphisme $\mathrm{T}_{\mathrm{Q}}\left(\mathbb{Z}_{p}\right) \simeq \Gamma_{\mathrm{Q}} \times \mathrm{T}_{\mathrm{Q}}(\mathbb{Z} / p)$. Soit $\mathbb{Z}_{p}\left[\left[\mathrm{~T}_{\mathrm{Q}}\left(\mathbb{Z}_{p}\right)\right]\right]$ l'algèbre de groupe complétée et son sous-anneau de serie formelle $\Lambda_{\mathrm{Q}}=\mathbb{Z}_{p}\left[\left[\Gamma_{\mathrm{Q}}\right]\right]$.

$\mathrm{L}^{\prime}$ action de $\mathrm{T}_{\mathrm{Q}}\left(\mathbb{Z}_{p}\right)$ sur $e_{\mathrm{P}} V_{m}^{S \mathrm{P}}$ induit une action continue sur $\mathcal{V}^{\text {ord-P }}$ et $V^{\text {ord-P, }, \star}$.

Le théorème qui suit est dû à Hida ([7], th. 1.1.) dans le cas où $\mathrm{P}=\mathrm{B}$.

ThÉORÈme 7.1 (contrôle vertical). - 1 . Pour tout poids $\kappa \in X\left(\mathrm{~T}_{\mathrm{Q}}\right)$, le module $e_{\mathrm{P}} V_{\text {cusp }, \infty}^{S \mathrm{P}}\left[-\kappa^{\prime}\right]$ est libre de rang fini sur $\mathbb{Z}_{p}$. Ce rang ne dépend que de la classe de $\kappa$ modulo $p-1$.

2. Si le poids $\kappa$ est dominant, on $a$ :

$$
e_{\mathrm{P}} \mathrm{H}^{0}\left(\mathcal{J}_{\infty, 1} / \mathrm{P}, \omega^{\kappa}\right) \simeq e_{\mathrm{P}} V_{\infty}^{S \mathrm{P}}\left[-\kappa^{\prime}\right] .
$$

3. Si le poids $\kappa$ est Q-régulier, on a :

$$
e_{\mathrm{P}} \mathrm{H}^{0}\left(\phi, \omega^{\kappa}\right) \simeq e_{\mathrm{P}} V_{\infty}^{S \mathrm{P}}\left[-\kappa^{\prime}\right] .
$$

4. Si le poids $\kappa$ est $\mathrm{Q}$-très régulier, on $a$ :

$$
e_{\mathrm{P}} \mathrm{S}\left(\kappa, \Gamma_{\mathcal{N}}, \mathbb{Z}_{p}\right) \simeq e_{\mathrm{P}} V_{\text {cusp }, \infty}^{S \mathrm{P}}\left[-\kappa^{\prime}\right] .
$$

5. Le module $\mathcal{V}_{\text {cusp }}^{\text {ord } \mathrm{P}, \star}$ est libre de rang fini sur $\Lambda_{\mathrm{Q}}$.

6. Pour tout poids $\kappa$ on a :

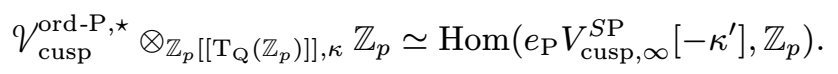

Si $\mathrm{P}=\mathrm{GL}_{g}$, tous les énoncés précédents sont valables sans supposer les formes cuspidales.

Démonstration. - Les points 1, 2, 3 et 4 sont la proposition 5.4, le théoreme 6.6 et le corollaire $5.2 \mathrm{du}$ texte.

Passons à la démonstration des points 5 et 6 . Tout caractère $\kappa \in X\left(\mathrm{~T}_{\mathrm{Q}}\right)$, on a un isomorphisme $V_{\text {cusp }}^{\text {ord- }}\left[-\kappa^{\prime}\right]=e_{\mathrm{P}} V_{\text {cusp }, \infty}^{S P}\left[-\kappa^{\prime}\right] \otimes_{\mathbb{Z}_{p}} \mathbb{Q}_{p} / \mathbb{Z}_{p}$. On a également un accouplement de dualité :

$$
\operatorname{Hom}_{\mathbb{Q}_{p} / \mathbb{Z}_{p}}\left(\mathcal{V}_{\text {cusp }}^{\text {ord-P }}\left[-\kappa^{\prime}\right], \mathbb{Q}_{p} / \mathbb{Z}_{p}\right)=\mathcal{V}_{\text {cusp }}^{\text {ord- }, \star} \otimes_{\left.\mathbb{Z}_{p}\left[T_{Q}\left(\mathbb{Z}_{p}\right)\right]\right], \kappa} \mathbb{Z}_{p}
$$

Comme $\operatorname{Hom}_{\mathbb{Q}_{p} / \mathbb{Z}_{p}}\left(\mathcal{V}_{\text {cusp }}^{\text {ord-P }}\left[-\kappa^{\prime}\right], \mathbb{Q}_{p} / \mathbb{Z}_{p}\right)=\operatorname{Hom}\left(e_{\mathrm{P}} V_{\text {cusp }, \infty}^{S \mathrm{P}}\left[-\kappa^{\prime}\right], \mathbb{Z}_{p}\right)$ le point 6 est démontré. 
Soit $\chi: \mathrm{T}_{\mathrm{Q}}\left(\mathbb{Z}_{p}\right) \rightarrow(\mathbb{Z} / p \mathbb{Z})^{\times}$un caractère et $\tilde{\chi}: \mathrm{T}_{\mathrm{Q}}\left(\mathbb{Z}_{p}\right) \rightarrow \mathbb{Z}_{p}^{\times}$le relèvement de Teichmüller. Notons $d(\chi)$ le rang du $\mathbb{Z}_{p}$-module $\mathcal{V}_{\text {cusp }}^{\text {ord-P }, \star} \otimes_{\mathbb{Z}_{p}\left[\left[\mathrm{~T}_{\mathrm{Q}}\left(\mathbb{Z}_{p}\right)\right]\right], \tilde{\chi}} \mathbb{Z}_{p}$ et choisissons en une base $s_{1}, \ldots, s_{d(\chi)}$.

Considérons l'application $\Lambda_{\mathrm{Q}}$-linéaire,

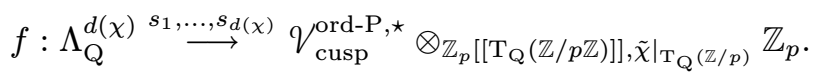

Par le lemme de Nakayama $f$ est surjective.

Pour tout caractère $\kappa \in X\left(\mathrm{~T}_{\mathrm{Q}}\right)$ tel que $\left.\kappa\right|_{\mathrm{T}_{\mathrm{Q}}(\mathbb{Z} / p \mathbb{Z})}=\left.\tilde{\chi}\right|_{\mathrm{T}_{\mathrm{Q}}(\mathbb{Z} / p)}$, l'application induite

$$
f \otimes 1: \Lambda_{\mathrm{Q}}^{d(\chi)} \otimes_{\Lambda_{\mathrm{Q}}, \kappa} \mathbb{Z}_{p} \rightarrow V_{\text {cusp }}^{\text {ord-P }, \star} \otimes_{\mathbb{Z}_{p}\left[\left[\mathrm{~T}_{\mathrm{P}}\left(\mathbb{Z}_{p}\right)\right]\right], \kappa} \mathbb{Z}_{p}
$$

est un isomorphisme car c'est une application linéaire surjective entre deux $\mathbb{Z}_{p}$-modules libres de même rang. Par densité Zariskienne des caractères $\kappa$ considérés dans Spec $\Lambda_{\mathrm{Q}}$, on en déduit que $f$ est un isomorphisme.

Comme

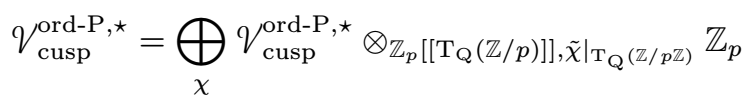

où la somme porte sur les caractères $\chi: \mathrm{T}_{\mathrm{P}}(\mathbb{Z} / p) \rightarrow \mathbb{Z} / p^{\times}$on en déduit le 5 .

Voici l'énoncé correspondant pour des formes avec un nebentypus. Soit $q$ un nombre premier ne divisant pas $N p, A$ l'anneau des entiers d'une extension finie $K$ de $\mathbb{Q}_{p}$ et $\chi:(\mathbb{Z} / q \mathbb{Z})^{\times} \rightarrow A^{\times}$un caractère. Notons $V_{1, q}^{\text {ord-P }}=\operatorname{colim}_{m \geq 0} e_{\mathrm{P}} V_{1, q, m}^{S \mathrm{P}}$ et $V_{1, q, \text { cusp }}^{\text {ord-P }}$ le sous-module des formes cuspidales. Soit $V_{1, q, \chi}^{\text {ord-P }, \star}$ et $V_{1, q, \chi, \text { cusp }}^{\text {ord-P }, \star}$ les duaux à valeur dans $K / A$ de $V_{1, q}^{\text {ord-P }}[\chi]$ et $V_{1, q, \text { cusp }}^{\text {ord-P }}[\chi]$.

ThÉORÈme 7.2. - 1. Pour tout poids $\kappa \in X\left(\mathrm{~T}_{\mathrm{Q}}\right)$, le module $e_{\mathrm{P}} V_{1, q, \mathrm{cusp}, \infty}^{S \mathrm{P}}\left[\chi,-\kappa^{\prime}\right]$ est libre de rang fini sur $\mathbb{Z}_{p}$. Ce rang ne dépend que de la classe de $\kappa$ modulo $p-1$.

2. Si le poids $\kappa$ est dominant, on a :

$$
e_{\mathrm{P}} \mathrm{H}^{0}\left(\left(\mathcal{J}_{0, q}\right)_{\infty, 1} / \mathrm{P}, \omega^{\kappa}(\chi)\right) \simeq e_{\mathrm{P}} V_{1, q, \infty}^{S \mathrm{P}}\left[\chi,-\kappa^{\prime}\right] .
$$

3. Si le poids $\kappa$ est Q-régulier, on a :

$$
e_{\mathrm{P}} \mathrm{H}^{0}\left(\phi_{0, q}, \omega^{\kappa}(\chi)\right) \simeq e_{\mathrm{P}} V_{\infty}^{S \mathrm{P}}\left[\chi,-\kappa^{\prime}\right] .
$$

4. Si le poids $\kappa$ est $\mathrm{Q}$-très régulier, on $a$ :

$$
e_{\mathrm{P}} \mathrm{S}\left(\kappa, \Gamma_{\mathcal{N}}^{0}(q), \chi, A\right) \simeq e_{\mathrm{P}} V_{1, q, \operatorname{cusp}, \infty}^{S \mathrm{P}}\left[\chi,-\kappa^{\prime}\right] .
$$

5. Le module $V_{1, q, \chi, \text { cusp }}^{\text {ord-P, }}$ est libre de rang fini sur $\Lambda_{\mathrm{Q}} \hat{\otimes}_{\mathbb{Z}_{p}} A$.

TOME $140-2012-\mathrm{N}^{\mathrm{O}} 3$ 
6. Pour tout poids $\kappa$ on $a$ :

$$
V_{1, q, \chi, \text { cusp }}^{\text {ord-P }, \star} \otimes_{A\left[\left[\mathrm{~T}_{\mathrm{Q}}\left(\mathbb{Z}_{p}\right)\right]\right], \kappa} A \simeq \operatorname{Hom}\left(e_{\mathrm{P}} V_{1, q, \operatorname{cusp}, \infty}^{S \mathrm{P}}\left[\chi,-\kappa^{\prime}\right], A\right) .
$$

Si $\mathrm{P}=\mathrm{GL}_{g}$, tous les énoncés précédents sont valables sans supposer les formes cuspidales.

\section{La théorie des familles de Hida}

Nous allons appliquer le théorème de contrôle vertical à l'étude de l'algèbre de Hecke.

DÉfinition 8.1. - $\quad$ 1. L'algèbre de Hecke sphérique $\mathcal{H}_{\ell}$ en un nombre premier $\ell$, est l'algèbre

$$
\mathbb{Z}\left[\mathrm{G}\left(\mathbb{Q}_{\ell}\right) / / \mathrm{G}\left(\mathbb{Z}_{\ell}\right)\right]
$$

des fonctions à support compact sur $\mathrm{G}\left(\mathbb{Q}_{\ell}\right)$, bi-invariantes sous l'action de $\mathrm{G}\left(\mathbb{Z}_{\ell}\right)$, munie du produit de convolution.

2. Pour tout entier $N$, on note $\mathcal{H}^{N}$ le produit tensoriel restreint des algèbres de Hecke $\mathcal{H}_{\ell}$ pour les nombres premiers $\ell$ ne divisant pas $N$.

On rappelle que ces algèbres sont commutatives [19].

Proposition 8.1. - Soit $\mathrm{P}$ un parabolique standard de $\mathrm{GL}_{g}, \mathrm{Q}=\omega_{0} \cdot{ }^{t} \mathrm{P} . \omega_{0}$. Il existe une action naturelle de $\mathcal{H}^{N p}$ sur $V_{\mathrm{cusp}, \infty}^{S \mathrm{P}}$. Cette action commute $\grave{a}$ l'action de $\mathrm{T}_{\mathrm{Q}}\left(\mathbb{Z}_{p}\right)$ et au projecteur $e_{\mathrm{P}}$ de $\mathrm{P}$-ordinarité.

Démonstration. - Dans [3], VII, 3. on associe à toute double classe $\mathrm{G}\left(\mathbb{Z}_{\ell}\right) \beta \mathrm{G}\left(\mathbb{Z}_{\ell}\right)$ avec $\beta \in \mathrm{G}\left(\mathbb{Q}_{\ell}\right)$ une correspondance algébrique $X_{\beta}$ au dessus de $X$. Les deux projections sont étales et finies. On peut restreindre cette correspondance au lieu ordinaire de $X, \phi_{\infty} \times_{\bar{X}} X$. Comme $\ell$ est différent de $p$, on voit facilement que cette correspondance se prolonge à la tour d'Igusa non compactifiée $\mathcal{T}_{\infty}^{\text {nc }}$. On obtient donc une action de $\mathscr{H}_{\ell}$ (voir [3], p. 254 pour la compatibilité à la structure d'algèbre) sur les fonctions régulières sur la tour d'Igusa non compactifié qui sont aussi, d'après le principe de Koecher, les fonctions sur la tour d'Igusa. Cette action commute avec l'action de $\mathrm{GL}_{g}\left(\mathbb{Z}_{p}\right)$. Ceci permet bien de définir une action de $\mathcal{H}^{N p}$ sur $V_{\infty}^{S \mathrm{P}}$. Cette action respecte la cuspidalité car une somme de fonctions cuspidales est cuspidale. Elle commute à l'action de tout opérateur de Hecke de niveau $p$ et donc au projecteur $e_{\mathrm{P}}$. 
On obtient donc une action $\mathbb{Z}_{p}\left[\left[\mathrm{~T}_{\mathrm{Q}}\left(\mathbb{Z}_{p}\right)\right]\right]$-linéaire de $\mathcal{H}^{N p}$ sur le $\mathbb{Z}_{p}\left[\left[\mathrm{~T}_{\mathrm{Q}}\left(\mathbb{Z}_{p}\right)\right]\right]$-module de rang fini $\mathcal{V}_{\text {cusp }}^{\text {ord- }, \star}$. Ce module est libre de rang fini comme $\Lambda_{\mathrm{Q}}$-module. Soit $\mathbb{T}^{\text {ord-P }}$ la $\mathbb{Z}_{p}\left[\left[\mathrm{~T}_{\mathrm{Q}}\left(\mathbb{Z}_{p}\right)\right]\right]$-algèbre engendrée par l'image de $\mathscr{H}^{N p}$ dans $\operatorname{End}_{\mathbb{Z}_{p}\left[\left[\mathrm{~T}_{\mathrm{Q}}\left(\mathbb{Z}_{p}\right)\right]\right]}\left(\mathcal{V}_{\text {cusp }}^{\text {ord- }, \star}\right)$. C'est une $\Lambda_{\mathrm{Q}}$-algèbre finie, sans torsion comme $\Lambda_{\mathrm{Q}}$-module.

Pour tout caractère $\kappa \in X\left(\mathrm{~T}_{\mathrm{Q}}\right)$, les $\overline{\mathbb{Q}}_{p}$ points de Spec $\mathbb{T}^{\text {ord-P }} \otimes_{\mathbb{Z}_{p}\left[\left[\mathrm{~T}_{\mathrm{Q}}\left(\mathbb{Z}_{p}\right)\right]\right], \kappa}$ $\mathbb{Z}_{p}$ sont en bijection avec les systèmes de valeurs propres pour l'algèbre $\mathcal{H}^{N p}$ agissant sur $e_{\mathrm{P}} V_{\text {cusp }, \infty}^{S \mathrm{P}}\left[-\kappa^{\prime}\right]$.

Grâce au relèvement de Teichmüller, on a canoniquement $\mathrm{T}_{\mathrm{Q}}\left(\mathbb{Z}_{p}\right)=\Gamma_{\mathrm{Q}} \times$ $\mathrm{T}_{\mathrm{Q}}(\mathbb{Z} / p \mathbb{Z})$. Tout caractère $\chi: \mathrm{T}_{\mathrm{Q}}(\mathbb{Z} / p \mathbb{Z}) \rightarrow \mathbb{Z}_{p}^{\times}$définit donc un morphisme $\chi: \mathbb{Z}_{p}\left[\left[\mathrm{~T}_{\mathrm{Q}}\left(\mathbb{Z}_{p}\right)\right]\right] \rightarrow \Lambda_{\mathrm{Q}}$. Le schéma Spec $\mathbb{Z}_{p}\left[\left[\mathrm{~T}_{\mathrm{Q}}\left(\mathbb{Z}_{p}\right)\right]\right]$ est semi-local. C'est la réunion, indexée par les caractères $\chi: \mathrm{T}_{\mathrm{Q}}(\mathbb{Z} / p \mathbb{Z}) \rightarrow \mathbb{Z}_{p}^{\times}$, des schémas locaux $\operatorname{Spec} \Lambda_{\mathrm{Q}} \stackrel{\chi}{\hookrightarrow} \operatorname{Spec} \mathbb{Z}_{p}\left[\left[\mathrm{~T}_{\mathrm{Q}}\left(\mathbb{Z}_{p}\right)\right]\right]$.

Pour tout caractère $\chi: \mathrm{T}_{\mathrm{Q}}(\mathbb{Z} / p \mathbb{Z}) \rightarrow \mathbb{Z}_{p}^{\times}$, notons

$$
\mathbb{T}_{\chi}^{\text {ord-P }}=\mathbb{T}^{\text {ord-P }} \otimes_{\mathbb{Z}_{p}\left[\left[\mathrm{~T}_{\mathrm{Q}}\left(\mathbb{Z}_{p}\right)\right]\right], \chi} \Lambda_{\mathrm{Q}}
$$

On a donc un carré cartésien :

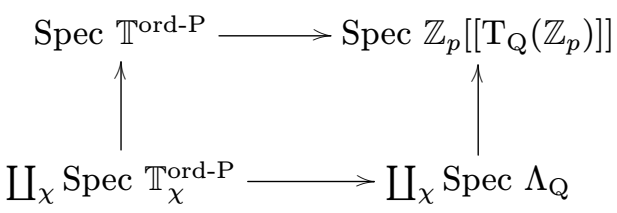

ThÉORÈme 8.1. - Pour tout caractère $\chi: \mathrm{T}_{\mathrm{Q}}(\mathbb{Z} / p \mathbb{Z}) \rightarrow \mathbb{Z}_{p}^{\times}$, le morphisme

$$
f: \operatorname{Spec} \mathbb{T}_{\chi}^{\text {ord-P }} \rightarrow \operatorname{Spec} \Lambda_{\mathrm{Q}}
$$

est fini et toute composante irréductible de Spec $\mathbb{T}_{\chi}^{\text {ord-P }}$ se surjecte sur $\operatorname{Spec} \Lambda_{\mathrm{Q}}$.

Démonstration. - Soit $x \in \operatorname{Spec} \mathbb{T}_{\chi}^{\text {ord-P }}$ tel que $f(x)$ n'est pas le point générique de $\operatorname{Spec} \Lambda_{\mathrm{Q}}$. Soit $y^{\prime}$ une générisation de $f(x)$. On va vérifier que $x$ admet une générisation $y$ tel que $f(y)=y^{\prime}$. En effet, le $\Lambda_{\mathrm{Q}^{-}}$module $\mathbb{T}_{\chi}^{\text {ord-P }}$ est sans torsion. Localisons en $x$ et $f(x)$. Le module ( $\left.\mathbb{T}_{\chi}^{\text {ord-P }}\right)_{x}$ est un $\left(\Lambda_{\mathrm{Q}}\right)_{f(x)^{-m o d u l e}}$ sans torsion. La fibre au dessus de $y^{\prime},\left(\mathbb{T}_{\chi}^{\text {ord-P }}\right)_{k\left(y^{\prime}\right)}$ est non nulle, elle possède donc un point $y$.

Corollaire 8.1. - Soit $\kappa \in X\left(\mathrm{~T}_{\mathrm{Q}}\right)$. Soit $f \in e_{\mathrm{P}} V_{\infty \text {,cusp }}^{S \mathrm{P}}\left[-\kappa^{\prime}\right] \otimes_{\mathbb{Z}_{p}} \overline{\mathbb{Z}}_{p}$ une forme modulaire p-adique cuspidale $\mathrm{P}$-ordinaire et propre pour l'action de $\mathcal{H}^{N p}$.

1. Pour tout poids $\tilde{\kappa} \in X\left(\mathrm{~T}_{\mathrm{Q}}\right)$, congru à $\kappa$ modulo $p-1$, il existe une forme $\tilde{f} \in e_{\mathrm{P}} V_{\infty, \text { cusp }}^{S \mathrm{P}}\left[-\tilde{\kappa}^{\prime}\right] \otimes_{\mathbb{Z}_{p}} \overline{\mathbb{Z}}_{p}$, propre pour $\mathcal{H}^{N p}$, de système de valeurs propres congru à celui de $f$ modulo l'idéal maximal de $\overline{\mathbb{Z}}_{p}$.

TOME $140-2012-\mathrm{N}^{\circ} 3$ 
2. Il existe une suite $\left(\kappa_{n}\right)_{n \in \mathbb{N}} \in X\left(\mathrm{~T}_{\mathrm{Q}}\right)^{\mathbb{N}}$ de poids $\mathrm{Q}$-très réguliers convergeant vers $\kappa$ et des formes $f_{n} \in \mathrm{S}^{\text {ord-P }}\left(\kappa_{n}, \Gamma_{\mathcal{N}}, \overline{\mathbb{Z}}_{p}\right)$, propres pour l'action de $\mathcal{H}^{N p}$, telles que les systèmes de valeurs propres des $f_{n}$ convergent uniformément vers le système des valeurs propres de $f$.

Démonstration. - Il suffit de considérer une composante irréductible $C_{f}$ de Spec $\mathbb{T}^{\text {ord-P }}$ passant par $f$. Elle se surjecte sur une composante irréductible Spec $\Lambda_{\mathrm{Q}}$ de Spec $\mathbb{Z}_{p}\left[\left[\mathrm{~T}_{\mathrm{Q}}\left(\mathbb{Z}_{p}\right)\right]\right]$. Tout $\tilde{\kappa}$ congru modulo $p-1$ à $\kappa$ définit un point sur la même composante Spec $\Lambda_{\mathrm{Q}}$ que $\kappa$. On peut donc prendre $\tilde{f}$ un élément de la fibre de $C_{f}$ au dessus de $\tilde{\kappa}$.

De même, considérons une suite de poids $\left(\kappa_{n}\right)_{n \in \mathbb{N}}$, Q-très réguliers, convergeant vers $\kappa$. On peut alors trouver une suite de points $f_{n}$ au dessus de $\kappa_{n}$ qui converge vers $f$. Ces points correspondent bien à des formes modulaires arithmétiques car $\kappa_{n}$ est Q-très régulier.

REMARQUE 8.1. - Les composantes irréductibles de Spec $\mathbb{T}^{\text {ord-P }}$ sont appelées des familles de Hida de formes propres.

\section{Le théorème de contrôle horizontal}

Les techniques développées ici permettent de démontrer un théorème de contrôle horizontal qui a des applications à la construction des systèmes de Taylor-Wiles (voir [18]). Soit $q$ un nombre premier vérifiant $q=1 \bmod p$ et $q \nmid N$. Soit $A$ l'anneau d'entiers d'une extension finie de $\mathbb{Q}_{p}$. Soit $\pi$ une uniformisante de $A$.

Le groupe $(\mathbb{Z} / q \mathbb{Z})^{\times}$est le produit de son $p$-Sylow $\Delta_{q}$ par un groupe $\Delta_{q}^{\prime}$ d'ordre premier à $p$. Soit $\mathrm{P}$ un parabolique standard de $\mathrm{GL}_{g}, \mathrm{Q}=\omega_{0} \cdot{ }^{t} \mathrm{P} . \omega_{0}$ et $\kappa \in X\left(\mathrm{~T}_{\mathrm{Q}}\right)$. Notons comme précédemment $e_{\mathrm{P}} V_{1, q \text {,cusp }, \infty}^{S \mathrm{P}}\left[-\kappa^{\prime}\right]$ le $A$-module des formes de Siegel $p$-adiques pour le parabolique P, cuspidales, de niveau $\Gamma_{\mathcal{N}}^{1}(q)$, de poids $\kappa$, P-ordinaires. Le groupe $(\mathbb{Z} / q \mathbb{Z})^{\times}$agit sur ce module, pour tout caractère $\chi:(\mathbb{Z} / q \mathbb{Z})^{\times} \rightarrow A^{\times}$, on note $e_{\mathrm{P}} V_{1, q, \text { cusp }, \infty}^{S \mathrm{P}}\left[\chi,-\kappa^{\prime}\right]$ le sous-module constitué des éléments $\chi$-variants.

Soient $\chi, \chi^{\prime}:(\mathbb{Z} / q \mathbb{Z})^{\times} \rightarrow A^{\times}$deux caractères tels que $\left.\chi\right|_{\Delta_{q}^{\prime}}=\left.\chi^{\prime}\right|_{\Delta_{q}^{\prime}}$.

Proposition 9.1. - Les sous-modules $e_{\mathrm{P}} V_{1, q, \text { cusp }, \infty}^{S \mathrm{P}}\left[\chi,-\kappa^{\prime}\right] \otimes_{A} A / \pi \quad$ et $e_{\mathrm{P}} V_{1, q, \text { cusp }, \infty}^{S \mathrm{P}}\left[\chi^{\prime},-\kappa^{\prime}\right] \otimes_{A} A / \pi$ de $e_{\mathrm{P}} V_{1, q, \text { cusp }, \infty}^{S \mathrm{P}}\left[-\kappa^{\prime}\right] \otimes_{A} A / \pi$ sont égaux.

Démonstration. - D'après le théorème 6.4,

$$
\begin{aligned}
e_{\mathrm{P}} V_{1, q, \text { cusp }, \infty}^{S \mathrm{P}}\left[\chi,-\kappa^{\prime}\right] \otimes_{A} A / \pi & =e_{\mathrm{P}} V_{1, q, \text { cusp }, 1}^{S \mathrm{P}}\left[\chi,-\kappa^{\prime}\right] \\
& =e_{\mathrm{P}} V_{1, q, \text { cusp }, 1}^{S \mathrm{P}}\left[\chi^{\prime},-\kappa^{\prime}\right] \\
& =e_{\mathrm{P}} V_{1, q, \text { cusp }, \infty}^{S \mathrm{P}}\left[\chi^{\prime},-\kappa^{\prime}\right] \otimes_{A} A / \pi .
\end{aligned}
$$


Soit $K$ le corps des fractions de $A$. Considérons le module divisible

$$
V_{1, q, \kappa, \text { cusp }}^{\text {ord-P }}=\operatorname{colim}_{m \in \mathbb{N}} e_{\mathrm{P}} V_{1, q, \text { cusp }, m}^{S \mathrm{P}}\left[-\kappa^{\prime}\right]=e_{\mathrm{P}} V_{1, q, \text { cusp }, \infty}^{S \mathrm{P}}\left[-\kappa^{\prime}\right] \otimes_{A} K / A .
$$

Soit $V_{1, q, \kappa, \text { cusp }}^{\text {ord-P }}$ de dual de Pontryagin de $V_{1, q, \kappa, \text { cusp }}^{\text {ord-P }}$.

THÉORÈme 9.1 (contrôle horizontal). — Le $A\left[\Delta_{q}\right]$-module $V_{1, q, \kappa, \text { cusp }}^{\text {ord-P,^ }}$ est libre de rang fini.

De plus, pour tout caractère $\chi:(\mathbb{Z} / q \mathbb{Z})^{\times} \rightarrow A^{\times}$, on a

$$
\bigvee_{1, q, \kappa, \text { cusp }}^{\text {ord-P }} \otimes_{A\left[(\mathbb{Z} / q \mathbb{Z})^{\times}\right], \chi} A=\operatorname{Hom}_{A}\left(e_{\mathrm{P}} V_{1, q, \text { cusp }, \infty}^{S \mathrm{P}}\left[\chi,-\kappa^{\prime}\right], A\right) .
$$

En particulier, si le poids $\kappa$ est Q-très régulier,

$$
\mathcal{V}_{1, q, \kappa, \text { cusp }}^{\text {ord }, \mathrm{P}, \star} \otimes_{A[(\mathbb{Z} / q \mathbb{Z}) \times], \chi} A=\operatorname{Hom}_{A}\left(\mathrm{~S}^{\text {ord-P }}\left(\kappa, \Gamma_{\mathcal{N}}^{0}(q), \chi, A\right), A\right)
$$

Démonstration. — Par dualité on a

$$
V_{1, q, \kappa, \text { cusp }}^{\text {ord-P }} \otimes_{A\left[\mathbb{Z} / q^{\times}\right], \chi} A=\operatorname{Hom}_{A}\left(V_{1, q, \kappa, \text { cusp }}^{\text {ord-P }}[\chi], K / A\right)
$$

et l'identité découle de l'isomorphisme

$$
\operatorname{Hom}_{A}\left(V_{1, q, \kappa, \text { cusp }}^{\text {ord-P }}[\chi], K / A\right)=\operatorname{Hom}_{A}\left(e_{\mathrm{P}} V_{1, q, \text { cusp }, \infty}^{S \mathrm{P}}\left[\chi,-\kappa^{\prime}\right], A\right) .
$$

Reste à montrer la liberté.

Fixons un caractère $\chi_{0}: \Delta_{q}^{\prime} \rightarrow A^{\times}$. Soit $s_{1}, \ldots, s_{r}$ une base du $A$-module libre de rang fini :

$$
V_{1, q, \kappa, \text { cusp }}^{\text {ord-P }} \otimes_{A\left[\mathbb{Z} / q^{\times}\right], 1 \times \chi_{0}} A \text {. }
$$

Considérons le morphisme $A\left[\Delta_{q}\right]$-linéaire

$$
f: A\left[\Delta_{q}\right]^{r} \stackrel{s_{1} \cdots s_{r}}{\rightarrow} V_{1, q, \kappa, \text { cusp }}^{\text {ord } \mathrm{P}, \star} \otimes_{A\left[\Delta_{q}^{\prime}\right], \chi_{0}} A .
$$

Il est surjectif d'après le lemme de Nakayama et la proposition 9.1. Toujours d'après la proposition 9.1, pour tout caractère $\chi: \Delta_{q} \rightarrow A^{\times}$le morphisme induit :

$$
f \otimes 1: A\left[\Delta_{q}\right]^{r} \otimes_{A\left[\Delta_{q}\right], \chi} A \stackrel{s_{1} \cdots s_{r}}{\rightarrow} V_{1, q, \kappa, \text { cusp }}^{\text {ord- }, \star} \otimes_{A\left[\mathbb{Z} / q^{\times}\right], \chi \times \chi_{0}} A
$$

est un isomorphisme.

C'est donc que $f$ est un isomorphisme.

Quitte à faire une extension fidèlement plate de l'anneau $A$, on peut supposer

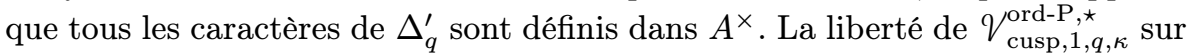
$A\left[\Delta_{q}\right]$ résulte de l'isomorphisme

$$
V_{1, q, \kappa, \text { cusp }}^{\text {ord-P }, \star} \stackrel{\sim}{\rightarrow} \bigoplus_{\chi_{0}: \Delta_{q}^{\prime} \rightarrow A^{\times}} V_{1, q, \kappa, \text { cusp }}^{\text {ord-P }, \star} \otimes_{A\left[\Delta_{q}^{\prime}\right], \chi_{0}} A .
$$

TOME $140-2012-\mathrm{N}^{\mathrm{O}} 3$ 


\section{Appendice A}

\section{Les correspondances $T_{p}$ et $U_{p}$}

Dans cet appendice, nous décrivons certaines propriétés fondamentales des opérateurs $T_{p}$ et $U_{p}$. Ces propriétés sont à la base de la théorie de Hida. Les résultats qui suivent sont bien connus des experts mais il n'y a pas, à notre connaissance, de référence écrite sur le sujet.

Rappelons quelques notations. Soit $p$ un nombre premier et $N \geq 3$ un entier premier à $p$. Soit $X$ la variété de Siegel de genre $g \geq 2$, de niveau $N$ sur Spec $\mathbb{Z}[1 / N]$. Soit $\mathscr{G} \stackrel{f}{\rightarrow} X$ le schéma abélien universel. Soit $\kappa=\left(k_{1}, \ldots, k_{g}\right) \in$ $\mathbb{Z}^{g}$ un $g$-uplet et soit $\omega^{\kappa}$ le faisceau des formes modulaires de poids $\kappa$ (voir 4.1.1).

On rappelle que pour tout $\mathbb{Z}[1 / N]$-module $A$ :

DÉfinition A.1. - Une forme de Siegel de niveau $N$ et de poids $\kappa$, à coefficients dans $A$ est un élément de $\mathrm{H}^{0}\left(X, \omega^{k} \otimes_{\mathbb{Z}} A\right)$. On note $\mathrm{M}(\kappa, N, A)$ ce module. On désigne également par $\mathrm{S}(\kappa, N, A)$ le sous-module des formes cuspidales.

Soit $X_{0}(p)$ le schéma de module dont les $R$-points sont les classes d'isomorphismes de :

- un schéma abélien $\mathscr{C} / R$.

- une polarisation principale $\lambda$.

- une structure de niveau $N$ notée $\psi_{N}$.

- un sous-groupe $H$ de $\mathscr{C}[p]$ lagrangien (c'est à dire totalement isotrope pour l'accouplement défini par la polarisation et l'accouplement de Weil et de rang $g$ ).

Sur $X_{0}(p)$ on dispose d'un schéma abélien universel $\mathscr{C}$ principalement polarisé, d'un sous-groupe lagrangien $H \subset \mathscr{C}[p]$, d'un second schéma abélien $\mathscr{G}^{\prime}=\mathscr{C} / H$ principalement polarisé et d'une isogénie de noyau $H$ :

$$
\pi: \mathscr{G} \rightarrow \mathscr{G}^{\prime}
$$

Notons $p_{1}, p_{2}: X(p) \rightarrow X$ les deux projections telles que $\mathscr{G} \times_{X, p_{1}} X(p)=\mathscr{G}$ et $\mathscr{G} \times_{X, p_{2}} X(p)=\mathscr{G}^{\prime}$. Elles sont finies et étales au dessus de Spec $\mathbb{Z}[1 / N p]$.

Le morphisme $\pi^{\star}: e^{\star} \Omega_{\mathscr{C}^{\prime} / X_{0}(p)}^{1} \rightarrow e^{\star} \Omega_{\mathscr{C} / X_{0}(p)}^{1}$ est un isomorphisme au dessus de $\mathbb{Z}[1 / N p]$. Il induit un isomorphisme de faisceaux :

$$
\pi^{\kappa}: p_{2}^{\star} \omega^{\kappa} \rightarrow p_{1}^{\star} \omega^{\kappa}
$$

pour tout poids $\kappa$. 
Pour tout $\mathbb{Z}[1 / N p]$-module, la correspondance $X_{0}(p)$ définit donc un endomorphisme $\tilde{T}_{p}$ du module $\mathrm{H}^{0}\left(X, \omega^{\kappa} \otimes_{\mathbb{Z}[1 / N p]} A\right)$ de la manière suivante :

$$
\begin{aligned}
\tilde{T}_{p}: \mathrm{H}^{0}\left(X, \omega^{\kappa} \otimes A\right) \rightarrow \mathrm{H}^{0}\left(X_{0}(p), p_{2}^{\star} \omega^{\kappa} \otimes A\right) & \\
& \rightarrow \mathrm{H}^{0}\left(X_{0}(p), p_{1}^{\star} \omega^{\kappa} \otimes A\right) \stackrel{\operatorname{Tr}_{p_{1}}}{\rightarrow} \mathrm{H}^{0}\left(X, \omega^{\kappa} \otimes A\right) .
\end{aligned}
$$

En fait, il faut normaliser cet opérateur pour obtenir l'opérateur de Hecke :

Définition A.2. - On définit l'opérateur de Hecke $T_{p}$ par la formule :

$$
T_{p}=p^{-\frac{g(g+1)}{2}} \tilde{T}_{p}
$$

De façon très concrète, si $f \in \mathrm{H}^{0}\left(X, \omega^{\kappa} \otimes A\right)$ on a

$$
\left.f\right|_{T_{p}}\left(\mathscr{C}, \lambda, \psi_{N}, \omega\right)=p^{-\frac{g(g+1)}{2}} \sum_{H \subset \mathscr{Q}[p]} f\left(\mathscr{C} / H, \lambda^{\prime}, \psi_{N}^{\prime}, \omega^{\prime}\right)
$$

où $H$ parcourt l'ensemble des sous-groupes lagrangiens de $\mathscr{Q}[p], \lambda^{\prime}$ est la polarisation induite par $\lambda^{p}, \psi_{N}^{\prime}$ est la structure de niveau principale image de $\psi_{N}, \omega$ et $\omega^{\prime}$ sont des trivialisations de faisceaux conormaux telles que $\pi^{\star} \omega^{\prime}=\omega$ avec $\pi: \mathscr{Q} \rightarrow \mathscr{Q} / H$ la projection.

On a une inclusion $\mathrm{M}\left(\kappa, N, \mathbb{Z}_{p}\right) \hookrightarrow \mathrm{M}\left(\kappa, N, \mathbb{Q}_{p}\right)$. On vient de définir l'action de $T_{p}$ sur $\mathrm{M}\left(\kappa, N, \mathbb{Q}_{p}\right)$, il est naturel de se demander quand $T_{p}$ préserve le sous-module $\mathrm{M}\left(\kappa, N, \mathbb{Z}_{p}\right)$.

La proposition suivante répond à cette question :

Proposition A.1. - Si le poids $\kappa=\left(k_{1}, \ldots, k_{g}\right)$ est tel que $k_{g} \geq g$, alors $T_{p}$ préserve le module $\mathrm{M}\left(\kappa, N, \mathbb{Z}_{p}\right)$.

La démonstration de cette proposition repose sur une étude géométrique de la correspondance $X_{0}(p)$ sur le lieu ordinaire, menée dans la partie A.1.

Pour tout $m$, soit $\phi_{m}^{\text {nc }} \rightarrow$ Spec $\mathbb{Z} / p^{m} \mathbb{Z}$ le lieu ordinaire de $X_{\mathbb{Z} / p^{m}}$. Soit $\phi_{\infty}^{\text {nc }} \rightarrow \operatorname{Spf} \mathbb{Z}_{p}$ le schéma formel obtenu par complétion de $X$ le long de $\phi_{1}^{\text {nc }}$ (L'abréviation «nc » signifie «non-compactifié ». On l'adjoint pour éviter toute confusion avec le texte principal où $\phi_{m}$ désigne le lieu ordinaire dans une compactification toroïdale).

La restriction au lieu ordinaire de $X_{0}(p)$ définit encore des correspondances que nous notons $\phi_{m}^{\text {nc }}(p)$ et $\phi_{\infty}^{\text {nc }}(p)$. Les deux projections restent étales et finies. Le rang multiplicatif du sous-groupe universel $H$ au dessus de $\phi_{\infty}^{\text {nc }}(p)$ est une fonction localement constante à valeurs entières comprises entre 0 et $g$. Notons $\phi_{\infty}^{\text {nc }}(g)^{=r}$ la réunion des composantes connexes où $H$ est de rang multiplicatif $r$.

Au dessus de $\phi_{\infty}^{\text {nc }}(p)^{=0}$, l'isogénie universelle $: \pi: \mathscr{G} \rightarrow \mathscr{G}^{\prime}$ est étale et induit un isomorphisme :

$$
\pi^{\star}: e^{\star} \Omega_{\mathscr{Q}^{\prime} / \phi_{\infty}^{\mathrm{nc}}(p)=0}^{1} \rightarrow e^{\star} \Omega_{\mathscr{C} / \oint_{\infty}^{\mathrm{nn}}(p)=0}^{1} .
$$

TOME $140-2012-\mathrm{N}^{\circ} 3$ 
Ceci permet de définir à nouveau un isomorphisme $\pi^{\kappa}: p_{2}^{\star} \omega^{\kappa} \rightarrow p_{1}^{\star} \omega^{\kappa}$.

On a un morphisme de trace :

$$
\operatorname{Tr}_{p_{1}}:\left(p_{1}\right)_{\star} \theta_{\phi_{\infty}^{\text {nc }}(p)=0} \rightarrow \theta_{\phi_{\infty}^{\text {nc }}} .
$$

Sa normalisation $p^{-\frac{g(g+1)}{2}} \operatorname{Tr}_{p_{1}}$ est encore bien définie (voir le corollaire A.1). On définit alors l'opérateur $U_{p}$ comme la composée :

$$
\begin{aligned}
U_{p}: \mathrm{H}^{0}\left(\phi_{\infty}^{\mathrm{nc}}, \omega^{\kappa}\right) \rightarrow \mathrm{H}^{0}\left(\phi_{\infty}^{\mathrm{nc}}(p)^{=0}, p_{2}^{\star} \omega^{\kappa}\right) & \\
& \rightarrow \mathrm{H}^{0}\left(\phi_{\infty}^{\mathrm{nc}}(p)^{=0}, p_{1}^{\star} \omega^{\kappa}\right) \stackrel{p^{-\frac{g(g+1)}{2}} \stackrel{\operatorname{Tr}_{p_{1}}}{\longrightarrow} \mathrm{H}^{0}\left(\phi_{\infty}^{\mathrm{nc}}, \omega^{\kappa}\right)}{ }
\end{aligned}
$$

On a une application de restriction :

$$
\mathrm{M}\left(\kappa, N, \mathbb{Z}_{p}\right) \hookrightarrow \mathrm{H}^{0}\left(\phi_{\infty}^{\text {nc }}, \omega^{\kappa}\right)
$$

C'est un problème classique de caractériser l'image de ce morphisme.

Soit $\bar{X}$ une compactification torö̈dale de $X([3])$. Notons $\overline{\mathfrak{X}}$ la complétion formelle de $\bar{X}$ le long de sa fibre rigide et $\bar{X}_{\text {rig }}$ sa fibre générique qui est un espace rigide-analytique quasi-compact. Soit $\phi_{\infty}$ l'ouvert d'ordinarité de $\overline{\mathfrak{X}}$. Notons $\bar{\phi}_{\text {rig }}$ la fibre rigide de $\phi_{\infty}$.

DÉfinition A.3. - L'espace des formes modulaires surconvergentes de niveau $N$ premier à $p$ et de poids $\kappa$ est :

$$
\mathrm{H}^{0}\left(\bar{X}, \omega^{\kappa}\right)^{\dagger}=\operatorname{colim}_{U} \mathrm{H}^{0}\left(U, \omega^{\kappa}\right)
$$

où $U$ parcourt l'ensemble des voisinages stricts de $\phi_{\text {rig }}$ dans $\bar{X}_{\text {rig }}$.

On a alors des inclusions :

$$
\mathrm{M}\left(\kappa, N, \mathbb{Q}_{p}\right) \hookrightarrow \mathrm{H}^{0}\left(\bar{X}, \omega^{\kappa}\right)^{\dagger} \hookrightarrow \mathrm{H}^{0}\left(\phi_{\text {rig }}, \omega^{\kappa}\right) .
$$

D'après le principe de Koecher, $\mathrm{H}^{0}\left(\phi_{\infty}^{\text {nc }}, \omega^{\kappa}\right)=\mathrm{H}^{0}\left(\phi_{\infty}, \omega^{\kappa}\right)$. Par quasicompacité, on a de plus $\mathrm{H}^{0}\left(\phi_{\infty}, \omega^{\kappa}\right) \otimes_{\mathbb{Z}_{p}} \mathbb{Q}_{p}=\mathrm{H}^{0}\left(\phi_{\text {rig }}, \omega^{\kappa}\right)$. On a donc une action de l'opérateur $U_{p}$ sur ce module.

Remarque A.1. - L'opérateur $U_{p}$ n'est autre que l'opérateur $T_{\beta_{0}}^{1}$ du numéro 5.1.3.

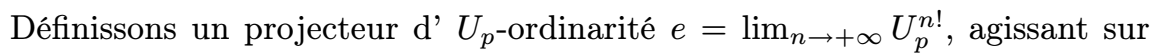
$\mathrm{H}^{0}\left(\phi_{\infty}, \omega^{\kappa}\right), \mathrm{H}^{0}\left(\phi_{\text {rig }}, \omega^{\kappa}\right)$. Notons enfin $\mathrm{H}_{\text {cusp }}^{0}\left(\phi_{\infty}, \omega^{\kappa}\right)$ et $\mathrm{H}_{\text {cusp }}^{0}\left(\phi, \omega^{\kappa}\right)$ les sousmodules des formes cuspidales de ces deux espaces.

ThÉorème A.1. - $\quad$ 1. Pour tout $\kappa$, le module $e \mathrm{H}_{\text {cusp }}^{0}\left(\varnothing_{\infty}, \omega^{\kappa}\right)$ est de rang fini.

2. Pour tout $t \gg 0$, on a des isomorphismes :

$$
e \mathrm{~S}\left(\kappa+\underline{t}, N, \mathbb{Z}_{p}\right) \rightarrow e \mathrm{H}_{\text {cusp }}^{0}\left(\varnothing_{\infty}, \omega^{\kappa+\underline{t}}\right)
$$


3. Toute section du module $e \mathrm{H}_{\text {cusp }}^{0}\left(\phi_{\text {rig }}, \omega^{\kappa}\right)$ surconverge.

4. Lorsque $\kappa$ est parallèle, les mêmes résultats valent sans supposer la cuspidalité.

La démonstration des points 1 et 2 repose sur une étude géométrique, menée au numéro A.2, de l'opérateur $T_{p}$ agissant sur une strate non-ordinaire; elle est achevée au numéro A.3. La démonstration du point 3 utilise la théorie du sousgroupe canonique de [1]. Elle fait l'objet du numéro A.4.

Nous nous sommes restreint au cas $g \geq 2$ pour pouvoir librement utiliser le principe de Koecher. Néanmoins nos arguments sont aussi valable pour $g=$ 1. Ils permettent de donner une nouvelle démonstration du résultat suivant, dû à Jochnowitz. Posons provisoirement $g=1$ et désignons par $X$ la courbe modulaire de niveau $N$ premier à $p$. Notons $\bar{X}$ sa compactification naturelle et $\phi_{\infty}$ le sous-schéma formel ordinaire de $\bar{X}$. Pour tout entier $k, \omega^{k}$ est le faisceau modulaire standard de poids $k$.

ThÉORÈme A.2 ([10]). — Pour $g=1$, le morphisme e $\mathrm{M}\left(k, N, \mathbb{Z}_{p}\right) \rightarrow$ $e \mathrm{H}^{0}\left(\phi_{\infty}, \omega^{k}\right)$ est un isomorphisme si $k \geq 3$.

Nos estimations ne permettent pas pour l'instant d'obtenir un résultat quantitatif de ce type pour $g \geq 2$. Il doit néanmoins être possible de les améliorer et d'obtenir des résultats précis lorsque $g=2$ et 3 et le poids est diagonal. Nous y reviendrons dans un prochain travail.

Soit $\mathcal{J}_{\infty, 1} \rightarrow \phi_{\infty}$ le premier cran de la tour d'Igusa. Pour tout parabolique standard $\mathrm{P}$ de $\mathrm{GL}_{g}$, on peut considérer le quotient $\mathcal{J}_{\infty, 1} / \mathrm{P}$ de la tour d'Igusa par $\mathrm{P}(\mathbb{Z} / p \mathbb{Z})$. On a rencontré dans le texte principal le module $\mathrm{H}^{0}\left(\mathcal{J}_{\infty, 1} / \mathrm{P}, \omega^{\kappa}\right)$ (voir thm. 7.1 par exmple). On dispose d'un plongement naturel $\mathrm{H}^{0}\left(\phi_{\infty}, \omega^{\kappa}\right) \hookrightarrow$ $\mathrm{H}^{0}\left(\mathcal{J}_{\infty, 1} / \mathrm{P}, \omega^{\kappa}\right)$. De plus, l'opérateur $U_{p}$ agit bien sur $\mathrm{H}^{0}\left(\mathcal{J}_{\infty, 1} / \mathrm{P}, \omega^{\kappa}\right)$ (cette action est définie au numéro 5.1 .3 où l'opérateur $U_{p}$ est noté $\left.T_{\beta_{0}}^{1}\right)$.

Supposons le parabolique $\mathrm{P}$ associé à des entiers $\left(n_{i}\right)_{1<i<r}$ strictement positifs tels que $\sum_{i=1}^{r} n_{i}=g$. Au parabolique $\mathrm{P}$, on associe le $X$-schéma $X_{\mathrm{P}}$ (appelé parfois variété de Siegel de niveau parahorique $\mathrm{P}$ ). Il paramètre les schémas abéliens $\mathscr{C}$ de dimension $g$, munis d'une polarisation principale, d'une structure de niveau $N$ et d'un drapeau de sous-groupes $H_{1} \subset \cdots H_{i} \cdots \subset H_{r-1} \subset \mathscr{C}[p]$ où $H_{i}$ est de hauteur $\sum_{j=r-i}^{r} n_{j}$ et est totalement isotrope. D'après [21], il existe une compactification torö̈dale $\bar{X}_{\mathrm{P}}$ de $X_{\mathrm{P}}$. On note $\mathcal{T}_{\infty, 1}^{\prime} / \mathrm{P}$ le schéma formel obtenu par complétion de $\bar{X}_{\mathrm{P}}$ le long de l'ouvert de sa fibre spécial où le schéma semi-abélien $\mathscr{C}$ est ordinaire et où les groupes $H_{i}$ sont de type multiplicatifs.

Si la décomposition polyédrale utilisée pour définir $\bar{X}$ et $\bar{X}_{\mathrm{P}}$ est la même, on a un morphisme d'oubli $\bar{X}_{\mathrm{P}} \rightarrow \bar{X}$ qui est propre et génériquement fini étale. Dans ce cas, on a simplement $\mathcal{T}_{\infty, 1}^{\prime} / \mathrm{P} \simeq \mathcal{J}_{\infty, 1} / \mathrm{P}$. On a de toute façon $\mathrm{H}^{0}\left(\mathcal{J}_{\infty, 1} / \mathrm{P}, \omega^{\kappa}\right)=\mathrm{H}^{0}\left(\mathcal{J}_{\infty, 1}^{\prime} / \mathrm{P}, \omega^{\kappa}\right)$. Nous supposons dans la suite que 
$\mathcal{T}_{\infty, 1}^{\prime} / \mathrm{P} \simeq \mathcal{T}_{\infty, 1} / \mathrm{P}$, cas auquel on peut toujours se ramener en modifiant nos choix combinatoires. Notons $\overline{\mathfrak{X}}_{\mathrm{P}}$ le schéma formel associé à $\bar{X}_{\mathrm{P}}$ et $\bar{X}_{\text {rig, } \mathrm{P}}$ sa fibre générique. Notons $\mathcal{T}_{\text {rig, } 1} / \mathrm{P}$ l'espace rigide associé à $\mathcal{T}_{\infty, 1} / \mathrm{P}$.

DÉFInItion A.4. - L'espace des formes modulaires surconvergentes de niveau N.P et de poids $\kappa$ est :

$$
\mathrm{H}^{0}\left(\bar{X}_{\mathrm{P}}, \omega^{\kappa}\right)^{\dagger}=\operatorname{colim}_{U} \mathrm{H}^{0}\left(U, \omega^{\kappa}\right)
$$

où $U$ parcourt l'ensemble des voisinages stricts de $\mathcal{J}_{\text {rig, } 1} / \mathrm{P}$ dans $\bar{X}_{\text {rig, }, \mathrm{P}}$.

THÉORÈme A.3. - Le module $e \mathrm{H}_{\text {cusp }}^{0}\left(\mathcal{G}_{\text {rig, }, 1} / \mathrm{P}, \omega^{\kappa}\right)$ est de dimension finie et toute section du module $e \mathrm{H}_{\text {cusp }}^{0}\left(\mathcal{G}_{\mathrm{rig}, 1} / \mathrm{P}, \omega^{\kappa}\right)$ surconverge.

\section{A.1. La correspondance $X_{0}(p)$ sur le lieu ordinaire}

A.1.1. Modèles locaux. - Soit $R=W\left(\overline{\mathbb{F}}_{p}\right)\left[\left[T_{1}, \ldots, T_{g(g+1) / 2}\right]\right]$ l'anneau des séries formelles en $g(g+1) / 2$ indéterminées, à coefficient dans l'anneau des vecteurs de Witt de la clôture algébrique de $\mathbb{F}_{p}$. Pour $i=0, \ldots, g$ nous pouvons définir un morphisme d'anneau :

$$
\begin{aligned}
f_{i}: R & \rightarrow R \\
T_{j} & \mapsto\left(1+T_{j}\right)^{p}-1 \text { pour } j=0, \ldots, i(i+1) / 2 \\
T_{j} & \mapsto T_{j} \quad \text { pour } j=i(i+1) / 2+1, \ldots, g(g+1) / 2
\end{aligned}
$$

Nous aurons plus tard à nous servir du lemme suivant :

Lemme A.1. - Soit $\operatorname{Tr}_{f_{i}}: R \rightarrow R$ l'application de trace associée au morphisme fini $f_{i}$.

$$
\operatorname{Im} \operatorname{Tr}_{f_{i}} \subset p^{i(i+1) / 2} \cdot R
$$

A.1.2. Description locale du morphisme $p_{1}$. - Soit $x \in X\left(\overline{\mathbb{F}}_{p}\right)$ un point géométrique situé dans le lieu ordinaire de la fibre spéciale en $p$ de $X$.

Il correspond au point $x$ un schéma abélien principalement polarisé ordinaire $\mathscr{G}_{x}$ sur $\overline{\mathbb{F}}_{p}$. La polarisation induit un accouplement symplectique :

$$
\mathscr{G}_{x}[p] \times \mathscr{G}_{x}[p] \rightarrow \mu_{p}
$$

et comme $\mathscr{G}_{x}$ est ordinaire, il existe un isomorphisme symplectique :

$$
\mathscr{G}_{x}[p] \simeq \mu_{p}^{g} \times \mathbb{Z} / p \mathbb{Z}^{g} .
$$

Notons $Y$ l'ensemble des points $y \in X_{0}(p)\left(\overline{\mathbb{F}}_{p}\right)$ rendant commutatif le diagramme suivant :

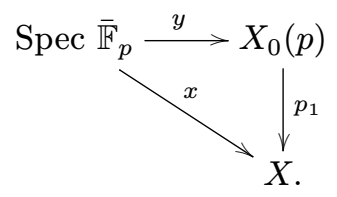


Il s'identifie à l'ensemble des sous-groupes $H \subset \mathscr{G}_{x}[p]$ lagrangiens.

Chaque tel sous-groupe est isomorphe à $\mu_{p}^{g-a} \times \mathbb{Z} / p^{a}$. Définissons l' entier $i(H)=a$, c'est le rang étale de $H$. Si à $y \in \mathcal{Y}$ correspond $H$, on écrira aussi $i(y)=i(H)$.

Pour tout schéma $S$ et point géométrique $s$ on note $S_{(s)}$ l'hensélisé strict de $S$ au point $s$ et $\hat{S}_{(s)}$ la complétion de $S_{(s)}$ au point $s$.

Proposition A.2. - On a

$$
X_{0}(p) \times_{X, p_{1}} X_{(x)}=\coprod_{y \in \mathcal{Y}} X_{0}(p)_{(y)} .
$$

Démonstration. - Comme le morphisme $p_{1}$ est fini au voisinage du point $x$ la proposition résulte des propriétés classiques des anneaux henséliens.

Proposition A.3. - 1. Il existe des isomorphismes $\hat{X}_{(x)} \simeq \operatorname{Spec} R$ et pour tout $y \in \mathcal{Y}, \hat{X}_{0}(p)_{(y)} \simeq \operatorname{Spec} R$.

2. Soit $y \in \mathcal{Y}$. On peut de plus choisir les isomorphismes de manière à faire commuter le diagramme :

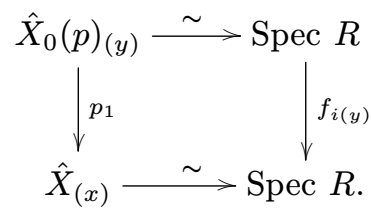

Démonstration. - Le premier point résulte de la lissité des espaces $X$ et $X_{0}(p)$ au voisinage des points considérés. Le second point résulte de la théorie de Serre-Tate ([11], théorème 2.1).

Corollaire A.1. - L'opérateur $U_{p}$ agissant sur $\mathrm{H}^{0}\left(\phi_{\infty}^{\mathrm{nc}}, \omega^{\kappa}\right)$ est bien défini.

Démonstration. - En effet, la normalisation de la trace est licite par le lemme A.1.

A.1.3. Intégralité de l'opérateur $T_{p}$. - Soit $y \in \mathcal{Y}$ et $\kappa=\left(k_{1}, \ldots, k_{g}\right)$ un poids. Commençons par décrire, au voisinage de $y$, l'image du morphisme $\pi^{\kappa}$ :

Lemme A.2. - On a

$$
\left.\left.\pi^{\kappa} p_{2}^{\star} \omega^{\kappa}\right|_{\hat{X}_{0}(p)_{(y)}} \subset p^{k_{g}(g-i(y))} \cdot p_{1}^{\star} \omega^{\kappa}\right|_{\hat{X}_{0}(p)_{(y)}} .
$$

TOME $140-2012-\mathrm{N}^{\mathrm{O}} 3$ 
Démonstration. - Fixons un isomorphisme de Spec $R \simeq \hat{X}_{0}(p)_{(y)}$. Soit la restriction de l'isogénie universelle $\left.\pi\right|_{\hat{X}_{0}(p)_{(y)}}: \mathscr{C}_{\hat{X}_{0}(p)_{(y)}} \rightarrow \mathscr{C}_{\hat{X}_{0}(p)_{(y)}}^{\prime}$. Elle induit une isogénie des groupes $p$-divisibles connexes. En choisissant des isomorphismes de ceux-ci avec $\mu_{p^{\infty}}^{g} / R$ on peut associer à $\pi$ une matrice dans $\mathrm{M}_{g}\left(\mathbb{Z}_{p}\right)$. Cette matrice est équivalente à la matrice :

$$
M=\left(\begin{array}{cc}
p .1_{g-i(y)} & 0 \\
0 & 1_{i(y)}
\end{array}\right) .
$$

Comme

$$
\left.\mathscr{L} i e \mathscr{C}\right|_{\hat{X}_{0}(p)_{(y)}}=\left.\mathscr{L} i e \mathscr{C}\left[p^{\infty}\right]^{0}\right|_{\hat{X}_{0}(p)_{(y)}}
$$

on en déduit l'existence d'un diagramme commutatif,

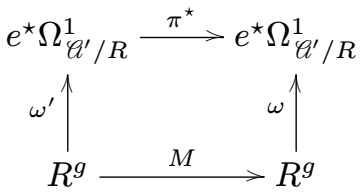

pour des trivialisations convenables $\omega$ et $\omega^{\prime}$. Soit $\left.f \in p_{2}^{\star} \omega^{\kappa}\right|_{\hat{X}_{0}(p)_{(y)}}$. Pour toute matrice $h \in \mathrm{GL}_{g}(R)$, on a $\left(\pi^{\kappa} f\right)(\omega \circ h)=f\left(\omega^{\prime} \circ M^{-1} \circ h\right)$. Par la décomposition d'Iwasawa, on a $M^{-1} . h=k . a . n$ avec $k \in \mathrm{GL}_{g}(R), a \in \mathrm{T}(R[1 / p]), n \in$ $\mathrm{U}^{0}(R[1 / p])$ et $v(\operatorname{det} a)=g-i(y)$. Comme $f\left(\omega^{\prime} \circ M^{-1} \circ g\right)=f(\omega \circ k) \kappa^{\prime}(a)$, on en déduit l'énoncé.

Concernant l'application $\operatorname{Tr}_{p_{1}}: H^{0}\left(\hat{X}_{0}(p)_{(y)}, p_{1}^{\star} \omega^{\kappa}\right) \rightarrow H^{0}\left(\hat{X}_{(x)}, \omega^{\kappa}\right)$, on montre :

LEMme A.3. - On a

$$
\operatorname{Im} \operatorname{Tr}_{p_{1}} \subset p^{i(i+1) / 2} \cdot H^{0}\left(\hat{X}_{(x)}, \omega^{\kappa}\right) .
$$

Démonstration. - Choisissant une trivialisation du faisceau $\omega^{\kappa}$ on se ramène au lemme A.1.

Proposition A.4. - Si le poids $\kappa$ est tel que $k_{g} \geq g$ alors $T_{p}$ préserve les structures entières.

Démonstration. - Soit $y \in \mathcal{Y}$. D'après les lemmes A.2 et A.3 on trouve que le composé :

$$
H^{0}\left(\hat{X}_{0}(p)_{(y)}, p_{2}^{\star} \omega^{\kappa}\right) \rightarrow H^{0}\left(\hat{X}_{0}(p)_{(y)}, p_{1}^{\star} \omega^{\kappa} \otimes_{Z} A\right) \stackrel{\operatorname{Tr}_{p_{1}}}{\rightarrow} H^{0}\left(\hat{X}_{(x)}, \omega^{\kappa}\right)
$$

est divisible par $p^{k_{g}(g-i(y))+i(y)(i(y)+1) / 2}$. Comme $\tilde{T}_{p}$ est au voisinage de $x$ la somme sur $\mathcal{Y}$ des opérateurs considérés et que $g(g+1) / 2 \leq(g-i(y)) g+$ $i(y)(i(y)+1) / 2$, on en déduit l'existence d'un opérateur bien défini $T_{p}$ agissant $\operatorname{sur} \mathrm{H}^{0}\left(\phi_{\infty}, \omega^{\kappa}\right)$. 
Soit $f \in \mathrm{M}\left(\kappa, N, \mathbb{Z}_{p}\right)$. Alors d'une part, $T_{p} . f \in \mathrm{M}\left(\kappa, N, \mathbb{Q}_{p}\right)$ et d'autre part $T_{p} .\left.f\right|_{\phi_{\infty}} \in \mathrm{H}^{0}\left(\phi_{\infty}, \omega^{\kappa}\right)$. Par densité du lieu ordinaire, on en déduit que $T_{p} . f \in$ $\mathrm{M}\left(\kappa, N, \mathbb{Z}_{p}\right)$.

On en déduit du même coup :

Corollaire A.2. - Si $k_{g}>g$ alors on a la congruence $U_{p}=T_{p} \bmod p$ comme opérateurs sur $\mathrm{H}^{0}\left(\phi_{\infty}, \omega^{\kappa}\right)$.

A.2. La correspondance $X_{0}(p)$ sur une strate non ordinaire. - Nous allons montrer la proposition suivante :

Proposition A.5. - Soit $\kappa$ un poids tel que $k_{g}>\frac{(p+1) g(g+1)}{2}$ et $f \in$ $\mathrm{M}\left(\kappa, N, \mathbb{Z}_{p}\right)$. Alors $T_{p} f \bmod p \in \mathrm{M}\left(\kappa, N, \mathbb{F}_{p}\right)$ est nulle sur le lieu supersingulier.

Remarque A.2. - Lorsque $g=1$, la proposition est exactement le lemme 3.2 de [10].

Soit $X^{1 / 2}$ le sous-schéma localement fermé de $X_{\mathbb{F}_{p}}$ des schémas abéliens ayant un polygone de Newton de pente 0 et 1 avec multiplicité $g-1,1 / 2$ avec multiplicité 2 . Il est dense dans le fermé non ordinaire d'après un résultat de F. Oort ([16], th. 3.1).

Soit $x \in X^{1 / 2}\left(\overline{\mathbb{F}}_{p}\right)$ et $\tilde{x} \in X\left(W\left(\overline{\mathbb{F}}_{p}\right)\right)$ un relèvement de $x$ (il existe bien par lissité de l'espace X). Soit $f \in \mathrm{M}\left(\kappa, N, \mathbb{Z}_{p}\right)$. Soit $\omega: W\left(\overline{\mathbb{F}}_{p}\right)^{g} \rightarrow e^{\star} \Omega_{\mathscr{C}_{\tilde{x}} / W\left(\overline{\mathbb{F}}_{p}\right)}^{1}$ une trivialisation.

On a

$$
\left.f\right|_{T_{p}}\left(\mathscr{G}_{\tilde{x}}, \omega\right)=p^{-\frac{g(g+1)}{2}} \sum_{H \subset \mathscr{C}_{\tilde{x}}[p]} f\left(\mathscr{G} / H, \omega^{\prime}\right)
$$

où la somme va sur les sous-groupes lagrangiens de $\mathscr{G}_{\tilde{x}}[p]\left(\overline{\mathbb{Q}}_{p}\right)$.

Rappelons dans ce cas la structure de $\mathscr{G}_{\tilde{x}}[p]$. Ce groupe est filtré :

$$
\mathscr{G}_{\tilde{x}}[p]^{\text {mult }} \subset \mathscr{G}_{\tilde{x}}[p]^{0} \subset \mathscr{G}_{\tilde{x}}[p] .
$$

Le premier cran est multiplicatif, le gradué $\mathscr{G}_{\tilde{x}}[p]^{0} / \mathscr{G}_{\tilde{x}}[p]^{m u l t}$ est un groupe bi-infinitésimal, isomorphe à la $p$-torsion d'une courbe elliptique à réduction supersingulière sur $W\left(\overline{\mathbb{F}}_{p}\right)$. Le gradué $\mathscr{G}_{\tilde{x}}[p] / \mathscr{G}_{\tilde{x}}[p]^{0}$ est étale.

La filtration est auto-duale pour la polarisation. En particulier, tout sous-groupe lagrangien $H \subset \mathscr{G}_{\tilde{x}}[p]\left(\overline{\mathbb{Q}}_{p}\right)$ a une image de rang 1 dans le gradué $\mathscr{G}_{\tilde{x}}[p]^{0} / \mathscr{G}_{\tilde{x}}[p]^{\text {mult }}\left(\overline{\mathbb{Q}}_{p}\right)$. L'adhérence schématique de cette image dans $\left(\mathscr{G}_{\tilde{x}}^{0} / \mathscr{G}_{\tilde{x}}^{m u l t}\right)_{\overline{\mathbb{Z}}_{p}}$ est alors un groupe de degré $\frac{1}{p+1}$ (au sens de [4]); autrement dit, il est isomorphe au schéma de Oort-Tate $G_{a, b}=\operatorname{Spec} \overline{\mathbb{Z}}_{p}[X] /\left(X^{p}-a X\right)$ avec $v(a)=\frac{1}{p+1}([22])$. 
Soit donc $H \subset \mathscr{G}_{\tilde{x}}[p]\left(\overline{\mathbb{Q}}_{p}\right)$ un sous-groupe lagrangien, de rang étale $i(H)$, de rang multiplicatif $g-1-i(H)$.

L'isogénie $\mathscr{C}_{\tilde{x}} \rightarrow \mathscr{C}_{\tilde{x}} / H$ est définie sur Spec $\overline{\mathbb{Z}}_{p}$.

Pour alléger les notations, posons simplement $\left(\mathscr{G}_{\tilde{x}}\right)_{\overline{\mathbb{Z}}_{p}}=\mathscr{G}$ et $\left(\mathscr{G}_{\tilde{x}}\right)_{\overline{\mathbb{Z}}_{p}} / H=\mathscr{G}^{\prime}$

Soit la matrice

$$
M=\left(\begin{array}{ccc}
p \cdot 1_{g-1-i(H)} & 0 & 0 \\
0 & p^{\frac{1}{p+1}} & 0 \\
0 & 0 & 1_{i(H)}
\end{array}\right) \in \mathrm{GL}_{g}\left(\overline{\mathbb{Q}}_{p}\right) .
$$

Il existe alors un diagramme commutatif

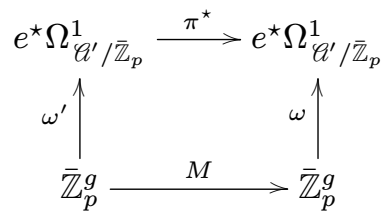

pour des trivialisations convenables $\omega$ et $\omega^{\prime}$. Un calcul analogue à celui de la démonstration du lemme A.2 montre alors :

LEMME A.4. - On a $\pi^{\kappa} p_{2}^{\star} \omega^{\kappa} \subset p^{k_{g}\left(g-1-i(H)+\frac{1}{p+1}\right)} p_{1}^{\star} \omega^{\kappa}$ au point $\left(\mathscr{G}, \mathscr{C}^{\prime}\right) d e$ $X_{0}(p)$.

Sous l'hypothèse $k_{g}>\frac{(p+1) g(g+1)}{2}$, on déduit de la formule définissant $T_{p}$ que pour toute trivialisation $\omega$,

$$
T_{p} f\left(\mathscr{G}_{\tilde{x}}, \omega\right) \in p W\left(\overline{\mathbb{F}}_{p}\right) .
$$

Il en résulte que $T_{p} f \bmod p$ est nulle sur le lieu $X^{1 / 2}$. Elle est alors nulle sur le lieu non ordinaire par densité de $X^{1 / 2}$ dans ce dernier.

A.3. Applications. - Le résultat suivant est dû à Igusa lorsque $g=1$. On peut le démontrer en général à l'aide de la théorie de Grothendieck-Messing et du théorème de densité de Oort.

ThÉORÈme A.4. - L'invariant de Hasse définit un fermé $V(H)$ de $X_{\mathbb{F}_{p}}$, d'ensemble sous-jacent le lieu non-ordinaire. Ce fermé est réduit.

Démonstration. - Soit $k$ un corps séparablement clos et $x \in X^{1 / 2}(k)$. On va montrer que l'invariant de Hasse $H$ définit une forme linéaire non nulle dans l'espace tangent de $X_{\mathbb{F}_{p}}$ au point $x$. Ceci montrera que $H$ est un paramètre régulier au point $x$. Par densité de $X^{1 / 2}$ dans le fermé de non-ordinarité (voir [16], th. 3.1), ceci montrera bien que $V(H)$ est réduit. 
Soit $k[\epsilon]$ l'algèbre des nombres duaux. Soit $\mathscr{G}_{x}$ le schéma abélien principalement polarisé associé à $x$. Comme $k$ est séparablement clos, le groupe $p$-divisible associé est le produit de ses parties multiplicatives, bi-infinitésimales et étales :

$$
\mathscr{G}_{x}\left[p^{\infty}\right]=\mathscr{G}_{x}\left[p^{\infty}\right]^{m} \times \mathscr{G}_{x}\left[p^{\infty}\right]^{00} \times \mathscr{G}_{x}\left[p^{\infty}\right]^{e t} .
$$

À partir de là, il est facile de décrire le cristal de Dieudonné $(\mathbb{D}, F)$ de $x$ évalué en $k[\epsilon] \rightarrow k$.

$\grave{A}$ isomorphisme près, on a $\mathbb{D}=\oplus_{i=1}^{2 g} k[\epsilon] e_{i}$ et la polarisation est donnée par la forme symplectique de matrice $J$. La matrice du Frobenius vaut

$$
\left(\begin{array}{ccc}
0_{g-1} & 0 & 0 \\
0 & A & 0 \\
0 & 0 & 1_{g-1}
\end{array}\right)
$$

avec $A$ la matrice $2 \times 2$,

$$
A=\left(\begin{array}{ll}
0 & 1 \\
0 & 0
\end{array}\right) .
$$

De plus sur $\mathbb{D} \otimes_{k[\epsilon]} k$, la filtration de Hodge est donné par : $\mathrm{Fil}^{1} \mathbb{D} \otimes_{k[\epsilon]} k=$ $\oplus_{i=1}^{g} k e_{i}$.

D'après la théorie de Grothendieck-Messing (voir [13]), le $k$-espace vectoriel $X_{x}(k[\epsilon])$ des relèvements de $x$ en un $k[\epsilon]$-point s'identifie à l'ensemble des modules facteurs directs, totalement isotropes $L \subset \mathbb{D}$ tels que $L \otimes_{k[\epsilon]} k=$ $\mathrm{Fil}^{1} \mathbb{D} \otimes_{k[\epsilon]} k$. À toute matrice $A \in \Sigma_{g}(k)\left(\Sigma_{g}\right.$ est le groupe des matrices symétriques de taille $g$ ), on associe l'espace $L_{A}$ engendré par les vecteurs colonnes :

$$
\operatorname{Col}(A)=\left(\begin{array}{c}
1_{g} \\
\omega_{0} A
\end{array}\right) .
$$

L'application $\Psi: A \mapsto L_{A}$ est un isomorphisme $\Psi: \Sigma_{g}(k) \simeq X_{x}(k[\epsilon])$. Pour tout $A \in \Sigma_{g}(k)$, l'application de Hasse-Witt du relèvement $\Psi(A)$ est l'application déduite de $F$,

$$
H W(A)=F: \mathbb{D} / L_{A} \rightarrow \mathbb{D} / L_{A} .
$$

Notons $\left(c_{i, j}\right)_{1 \leq i \leq 2 g, 1 \leq j \leq g}$ les coefficients de la matrice $\operatorname{Col}(A)$. L'image des vecteurs $e_{g+1}, \ldots, e_{2 g}$ dans $\mathbb{D} / L_{A}$ forme une base, notée $\left(\bar{e}_{i}\right)_{g+1 \leq i \leq 2 g}$ de ce module. Dans cette base, on a

$$
\begin{aligned}
F\left(\bar{e}_{g+1}\right) & =-\sum_{i=g+1}^{2 g} \epsilon \cdot c_{i, g} \cdot \bar{e}_{i} \\
F\left(\bar{e}_{j}\right) & =F\left(\bar{e}_{j}\right) \quad \text { si } j \geq g+2 .
\end{aligned}
$$

TOME $140-2012-\mathrm{N}^{\circ} 3$ 
Le déterminant de $H W(A)$ dans cette base vaut donc $H(A)=-\epsilon \cdot c_{g+1, g}$. La nullité de $H(A)$ est donnée par l'équation $c_{g+1, g}=0$ qui définit un hyperplan de $X_{x}(k[\epsilon])$.

Soit $\kappa$ un poids. D'après le théorème 6.2 , il existe $N(\kappa) \in \mathbb{N}$ tel que pour tout $t \geq N(\kappa)$, l'application de réduction :

$$
\mathrm{S}\left(\kappa+\underline{t}, N, \mathbb{Z}_{p}\right) \rightarrow \mathrm{S}\left(\kappa+\underline{t}, N, \mathbb{F}_{p}\right)
$$

est surjective.

Remarque A.3. - Lorsque le poids $\kappa$ est diagonal, on a de même la surjectivité de l'application de réduction :

$$
\mathrm{M}\left(\kappa+\underline{t}, N, \mathbb{Z}_{p}\right) \rightarrow \mathrm{M}\left(\kappa+\underline{t}, N, \mathbb{F}_{p}\right)
$$

ce qui permet dans ce cas d'étendre toute la discussion qui suit aux formes non cuspidales.

Si $t \geq \max \{g+1, N(\kappa)\}$, on a donc une action (définie par réduction modulo $p)$ de $T_{p}=U_{p}$ sur l'espace des formes modulaires cuspidales $\mathrm{S}\left(\kappa+\underline{t}, N, \mathbb{F}_{p}\right)$. On démontre aussi, par exemple par un calcul sur les schémas abéliens ordinaires, que la multiplication par l'invariant de Hasse,

$$
H: \mathrm{S}\left(\kappa+\underline{t}, N, \mathbb{F}_{p}\right) \rightarrow \mathrm{S}\left(\kappa+\underline{t+p-1}, N, \mathbb{F}_{p}\right)
$$

commute avec l'action de $U_{p}$.

Proposition A.6. - Si $t \geq \max \left\{\frac{g+1}{2}, N(\kappa), \frac{(p+1) g(g+1)}{2}-p+1\right\}$, la multiplication par l'invariant de Hasse :

$$
H: \mathrm{S}\left(\kappa+\underline{t}, N, \mathbb{F}_{p}\right) \rightarrow \mathrm{S}\left(\kappa+\underline{t+p-1}, N, \mathbb{F}_{p}\right)
$$

induit un isomorphisme :

$$
e \mathrm{~S}\left(\kappa+\underline{t}, N, \mathbb{F}_{p}\right) \stackrel{\sim}{\rightarrow} e \mathrm{~S}\left(\kappa+\underline{t+p-1}, N, \mathbb{F}_{p}\right)
$$

Démonstration. - En effet, tout élément de $e \mathrm{~S}\left(\kappa+t+p-1, N, \mathbb{F}_{p}\right)$ est nul sur le lieu supersingulier d'après la proposition A.5, donc divisible par l'invariant de Hasse d'après le théorème A.4.

Corollaire A.3. - Le module $e \mathrm{H}_{\text {cusp }}^{0}\left(\phi_{1}, \omega^{\kappa}\right)$ est de dimension finie, de plus pour $t \gg 0$, on a les égalités:

$$
e \mathrm{H}_{\text {cusp }}^{0}\left(\phi_{1}, \omega^{\kappa+t} \underline{t(p-1)}\right)=e \mathrm{~S}\left(\kappa+\underline{t(p-1)}, N, \mathbb{F}_{p}\right)
$$

et

$$
e \mathrm{H}_{\text {cusp }}^{0}\left(\phi_{\infty}, \omega^{\kappa+t \underline{t(p-1)}}\right)=e \mathrm{~S}\left(\kappa+\underline{t(p-1)}, N, \mathbb{Z}_{p}\right)
$$


Démonstration. - On a

$$
\mathrm{H}_{\text {cusp }}^{0}\left(\phi_{1}, \omega^{\kappa}\right)=\bigcup_{r \in \mathbb{Z}} \frac{\mathrm{S}\left(\kappa+\frac{r(p-1)}{H^{r}}, N, \mathbb{F}_{p}\right)}{H^{r}} .
$$

Comme la multiplication par l'invariant de Hasse,

$$
\mathrm{H}_{\text {cusp }}^{0}\left(\phi_{1}, \omega^{\kappa}\right) \rightarrow \mathrm{H}_{\text {cusp }}^{0}\left(\phi_{1}, \omega^{\kappa+\underline{p-1}}\right)
$$

est injective, on peut toujours remplacer $\kappa$ par $\kappa+t(p-1)$ pour $t \gg 0$. On a alors le droit d'écrire :

$$
e \mathrm{H}_{\text {cusp }}^{0}\left(\phi_{1}, \omega^{\kappa}\right)=\bigcup_{r \in \mathbb{Z}} \frac{e \mathrm{~S}\left(\kappa+\frac{r(p-1)}{H^{r}}, N, \mathbb{F}_{p}\right)}{H^{r}}=e \mathrm{~S}\left(\kappa, N, \mathbb{F}_{p}\right) .
$$

La dernière égalité vient de la proposition précédente.

On sait de plus que pour $t \gg 0$, les applications de réduction :

$$
\mathrm{H}_{\text {cusp }}^{0}\left(\phi_{\infty}, \omega^{\kappa+\underline{t}}\right) \rightarrow \mathrm{H}_{\text {cusp }}^{0}\left(\phi_{1}, \omega^{\kappa+\underline{t}}\right)
$$

et

$$
\mathrm{S}\left(\kappa+\underline{t}, N, \mathbb{Z}_{p}\right) \rightarrow \mathrm{S}\left(\kappa+\underline{t}, N, \mathbb{F}_{p}\right)
$$

sont surjectives. On en déduit alors le résultat final.

A.4. Complète continuité de $U_{p}$. - Notons $E \in \mathrm{H}^{0}\left(\bar{X}, \omega^{\underline{t}}\right)$ le relèvement de l'invariant de Hasse élevé à la puissance $t$. Soit $\overline{\mathfrak{X}}$ le schéma formel obtenu en complétant $\bar{X}$ le long de sa fibre spéciale. Notons $\bar{X}_{\text {rig }}$ sa fibre rigide. Soit $\phi_{\infty}$ l'ouvert formel ordinaire de $\overline{\mathfrak{X}}$. On note $\phi_{\text {rig }}$ sa fibre rigide. Pour tout $r \in \mathbb{Q}$, on définit les ouverts rigides $\bar{X}(r)=\left\{x \in X_{\text {rig }}, v(E(x)) \leq r\right\}$. On a $\bar{X}(0)=\phi_{\text {rig }}$ et l'ensemble $\left\{\bar{X}_{\text {rig }}(r), r \rightarrow 0^{+}\right\}$forme un système fondamental de voisinages stricts de $\phi_{\text {rig }}$ dans $\bar{X}_{\text {rig }}$.

D'après [12], prop. 2.4.1, les inclusions évidentes :

$$
\mathrm{H}^{0}\left(\bar{X}_{\text {rig }}(r), \omega^{\kappa}\right) \hookrightarrow \mathrm{H}^{0}\left(\bar{X}_{\text {rig }}(s), \omega^{\kappa}\right)
$$

pour $r>s \geq 0$ sont complètement continues si $r$ est suffisamment proche de 0 .

On a défini l'espace des formes surconvergentes :

$$
\mathrm{H}^{0}\left(\bar{X}, \omega^{\kappa}\right)^{\dagger}=\cup_{r>0} \mathrm{H}^{0}\left(\bar{X}_{\text {rig }}(r), \omega^{\kappa}\right) .
$$

On dispose d'un opérateur $U_{p}$ agissant sur $\mathrm{H}^{0}\left(\bar{X}(0), \omega^{\kappa}\right)$. La théorie du sousgroupe canonique de [1] (voir aussi [12]) nous permet d'étendre l'action de l'opérateur $U_{p}$ au module $\mathrm{H}^{0}\left(\bar{X}, \omega^{\kappa}\right)^{\dagger}$. Précisément, lorsque $r>0$ est suffisamment pretit, on a une application complètement continue :

$$
U_{p}: \mathrm{H}^{0}\left(\bar{X}_{\text {rig }}(r), \omega^{\kappa}\right) \rightarrow \mathrm{H}^{0}\left(\bar{X}_{\text {rig }}(p r), \omega^{\kappa}\right) \hookrightarrow \mathrm{H}^{0}\left(\bar{X}_{\text {rig }}(r), \omega^{\kappa}\right)
$$


Remarque A.4. - Soit $X_{\text {an }}$ l'analytifé de $X_{\mathbb{Q}_{p}}$ qui est un ouvert Zariskidense de $\bar{X}_{\text {rig. Notons }} X_{\text {an }}(r)=\bar{X}_{\text {rig }}(r) \cap X_{\text {an }}$. Dans [1] section 8, les auteurs définissent un relèvement surconvergent du Frobenius, $\Phi: X_{\text {an }}(r) \rightarrow X_{\text {an }}(p r)$ qui induirait donc plutôt une application :

$$
U_{p}: \mathrm{H}^{0}\left(X_{\text {an }}(r), \omega^{\kappa}\right) \rightarrow \mathrm{H}^{0}\left(X_{\text {an }}(p r), \omega^{\kappa}\right) \hookrightarrow \mathrm{H}^{0}\left(X_{\text {an }}(r), \omega^{\kappa}\right) .
$$

En effet, ils souhaitent utiliser la propriété modulaires de $X_{\text {an }}$ pour définit $\Phi$. Par contre dans [12] qui traite le cas des variétés de Hilbert, les auteurs définissent bien un relèvement surconvergent du Frobenius sur la compactification toroïdale (voir lem. 3.1.1 de loc. cit). Le point est que l'application sur la partie torique induite par le sous-groupe canonique est la multiplication par $p$ qui respecte les décompositions polyédrales. Dans le cas des variétés de Siegel, on peut montrer de la même manière que le relèvement surconvergent du Frobenius s'étend à la compactification toroïdale. Nous espérons revenir sur cette question prochainement. Par ailleurs, nous ignorons si l'application naturelle de restriction

$$
\mathrm{H}^{0}\left(\bar{X}_{\text {rig }}(r), \omega^{\kappa}\right) \rightarrow \mathrm{H}^{0}\left(X_{\text {an }}(r), \omega^{\kappa}\right)
$$

est un isomorphisme lorsque $r$ est proche de 0 . Elle est un isomorphisme lorsque $r=0$ par le principe de Koecher. On peut démontrer également qu'elle induit un isomorphisme pour les formes cuspidales, sur la partie ordinaire pour $U_{p}$, lorsque $r$ est proche de 0 .

D'après la théorie de Fredholm-Serre [20], on a, pour tout $r>0$ suffisamment petit, une décomposition topologique :

$$
\mathrm{H}^{0}\left(\bar{X}_{\text {rig }}(r), \omega^{\kappa}\right)=\bigoplus_{a \in \mathbb{R} \cup\{+\infty\}} \mathrm{H}^{0}\left(\bar{X}_{\text {rig }}(r), \omega^{\kappa}\right)[a]
$$

où $\mathrm{H}^{0}\left(\bar{X}_{\text {rig }}(r), \omega^{\kappa}\right)[a]$ est le lieu où l'opérateur $U_{p}-\lambda$ est topologiquement nilpotent pour $\lambda \in \overline{\mathbb{Q}}_{p}$ vérifiant $v(\lambda)=a$. Lorsque $a \in \mathbb{R}$, les espaces $\mathrm{H}^{0}\left(\bar{X}_{\text {rig }}(r), \omega^{\kappa}\right)[a]$ sont de dimension finie. De plus, il résulte du corollaire A.1 que $\mathrm{H}^{0}\left(\bar{X}_{\text {rig }}(r), \omega^{\kappa}\right)[a]$ est nulle si $a<0$. On dispose donc d'un idempotent bien défini, $e=\lim _{n \rightarrow \infty} U_{p}^{n !}$ agissant sur $\mathrm{H}^{0}\left(\bar{X}_{\text {rig }}(r), \omega^{\kappa}\right)$ et projetant sur $\mathrm{H}^{0}\left(\bar{X}_{\text {rig }}(r), \omega^{\kappa}\right)^{\dagger}[0]$.

Remarque A.5. - On peut montrer, par des techniques de prolongement analytique, que pour tout $a \in \mathbb{R}$, les espaces $\mathrm{H}^{0}\left(\bar{X}_{\text {rig }}(r), \omega^{\kappa}\right)[a]$ pour $r>0$ et proche de 0 , sont canoniquement isomorphes entre eux.

D'après le théorème 6.2 , on a

$$
\mathrm{H}_{\text {cusp }}^{0}\left(\phi_{\text {rig }}, \omega^{\kappa}\right)=\left\{\sum_{n=0}^{\infty} a_{n} E^{-n}, a_{n} \in \mathrm{H}^{0}\left(\bar{X}, \omega^{\kappa+\underline{t(p-1) n}}\right), \lim _{n \rightarrow \infty}\left|a_{n}\right|_{\phi_{\text {rig }}}=0\right\}
$$


où ||$_{\phi_{\text {rig }}}$ désigne la norme supremum sur $\phi_{\text {rig }}$.

On en déduit aussitôt que pour tout $r \in\left[0,+\infty\left[\right.\right.$, le module $\mathrm{H}_{\text {cusp }}^{0}\left(\bar{X}_{\text {rig }}(r), \omega^{\kappa}\right)$ est dense dans $\mathrm{H}_{\text {cusp }}^{0}\left(\phi_{\text {rig }}, \omega^{\kappa}\right)$. Fixons $r$ suffisamment petit tel que la théorie de Fredhom-Serre s'applique et le projecteur $e$ soit défini. Il en résulte que $e \mathrm{H}_{\text {cusp }}^{0}\left(\bar{X}_{\text {rig }}(r), \omega^{\kappa}\right)$ est dense dans $e \mathrm{H}_{\text {cusp }}^{0}\left(\phi_{\text {rig }}, \omega^{\kappa}\right)$. Comme $e \mathrm{H}_{\text {cusp }}^{0}\left(\bar{X}_{\text {rig }}(r), \omega^{\kappa}\right)^{\dagger}$ est un espace de dimension finie, on en déduit la proposition :

Proposition A.7. - Pour tout $\kappa$, on a

$$
e \mathrm{H}_{\text {cusp }}^{0}\left(\bar{X}, \omega^{\kappa}\right)^{\dagger}=e \mathrm{H}_{\text {cusp }}^{0}\left(\phi_{\text {rig }}, \omega^{\kappa}\right) \text {. }
$$

Pour tout parabolique standard $\mathrm{P}$ de $\mathrm{GL}_{g}$, on va raisonner de la même manière au niveau de la variété de Siegel parahorique $\bar{X}_{\mathrm{P}}$. On note $\overline{\mathfrak{X}}_{\mathrm{P}}$ le schéma formel obtenu par complétion de $\bar{X}_{\mathrm{P}}$ le long de sa fibre spéciale et $\bar{X}_{\text {rig,P }}$ sa fibre générique. On note $\mathcal{J}_{\infty, 1} / \mathrm{P}$ l'ouvert formel qui paramètre les schéma semi-abéliens ordinaires muni d'un drapeau de type multiplicatif. C'est le quotient par $\mathrm{P}(\mathbb{Z} / p \mathbb{Z})$ du premier cran d'une « compactification » de la tour d'Igusa. Sa fibre rigide est notée $\mathcal{J}_{\text {rig, }, 1} / \mathrm{P}$. On a définie le module des formes surconvergentes de niveau parahorique $\mathrm{P}$,

$$
\mathrm{H}^{0}\left(\bar{X}_{\mathrm{P}}, \omega^{\kappa}\right)^{\dagger}=\cup_{U} \mathrm{H}^{0}\left(U, \omega^{\kappa}\right)
$$

où $U$ parcourt l'ensemble des voisinages stricts de $\mathcal{J}_{\text {rig }, 1} / \mathrm{P}$ dans $\bar{X}_{\text {rig,P. }}$ La théorie du sous-groupe canonique de [1] (voir aussi [12]), permet d'étendre l'opérateur $U_{p}$ en un opérateur agissant sur le module $\mathrm{H}^{0}\left(\bar{X}_{\mathrm{P}}, \omega^{\kappa}\right)^{\dagger}$. On vérifie comme précédemment qu'il existe un projecteur $e=\lim _{n \rightarrow \infty} U_{p}^{n !}$ sur ce module, projetant vers un espace de dimension fini. Nous voulons à présent vérifier la densité des formes surconvergentes, cuspidales, de niveau parahorique $\mathrm{P}$ dans $\mathrm{H}_{\text {cusp }}^{0}\left(\mathcal{T}_{\infty, 1} / \mathrm{P}, \omega^{\kappa}\right)$.

D'après le chapitre 4 de [21], il existe une compactification minimale $X_{\mathrm{P}}^{\star}$ de $X_{\mathrm{P}}$ et une projection $\bar{X}_{\mathrm{P}} \rightarrow X_{\mathrm{P}}^{\star}$. Voici quelques faits importants. Soit $\mathscr{G} \rightarrow \bar{X}_{\mathrm{P}}$ le schéma semi-abélien étendant le schéma abélien universel et $H_{1} \subset$ $\cdots H_{i} \cdots \subset H_{r-1} \subset \mathscr{C}[p]$ les sous-groupes quasi-finis et plats étendant les sousgroupes universels. Posons $\mathscr{G}_{0}=\mathscr{G}$ et $\mathscr{G}_{i}=\mathscr{G} / H_{i}$. Soit pour tout $i$ le faisceau inversible $\omega_{i}=\Lambda^{g} e^{\star} \Omega_{\mathscr{G}_{i} / \bar{X}_{\mathrm{P}}}^{1}$. Formons $\omega=\otimes_{i=0}^{r-1} \omega_{i}$. C'est ce faisceau qui est utilisé dans [21], chap. 4, 4.2, pour définir la compactification minimale. En particulier, l'auteur montre que $\omega$ descend à la compactification minimale $X_{\mathrm{P}}^{\star}$ et définit un faisceau ample. Pour chaque $i$, on peut considérer l'invariant de Hasse $H_{i}$ du schéma semi-abélien $\left.\mathscr{C}_{i}\right|_{\mathbb{F}_{p}}$ qui définit une section de $\mathrm{H}^{0}\left(\bar{X}_{\mathrm{P}}, \mathbb{F}_{p} \otimes \omega_{i}\right)$. Le produit des $H_{i}$ est une section notée $H \in \mathrm{H}^{0}\left(\bar{X}_{\mathrm{P}}, \mathbb{F}_{p} \otimes \omega\right)$. Pour $t \in \mathbb{Z}$ suffisamment grand, la section $H^{t}$ se relève en une section $E$ de $\mathrm{H}^{0}\left(\bar{X}_{\mathrm{P}}, \omega^{t}\right)$. 
Le lieu de non annulation de la section $E$ est un ouvert d'image affine dans la compactification minimale. Voici le théorème dont nous avons besoin :

THÉORÈme A.5. - Le morphisme de changement de base :

$$
\mathrm{H}_{\text {cusp }}^{0}\left(\bar{X}_{\mathrm{P}}[1 / E], \omega^{\kappa}\right) \rightarrow \mathrm{H}_{\text {cusp }}^{0}\left(\bar{X}_{\mathrm{P}}[1 / E], \omega^{\kappa} \otimes_{\mathbb{Z}_{p}} \mathbb{Z} / p^{m} \mathbb{Z}\right)
$$

est surjectif.

La démonstration est mots pour mots celle du théorème 6.2.

Considérons $\bar{X}_{\mathrm{P}}^{\text {or }}$ le schéma formel obtenu en complétant $\bar{X}_{\mathrm{P}}$ le long du lieu ordinaire. Ce schéma formel est la réunion d'un nombre fini de composantes connexes. Le schéma formel $\mathcal{T}_{\infty, 1} / \mathrm{P}$ est la réunion de certaines de ces composantes. On a une suite d'applications :

$$
\mathrm{H}_{\text {cusp }}^{0}\left(\bar{X}_{\mathrm{P}}[1 / E], \omega^{\kappa}\right) \rightarrow \mathrm{H}_{\text {cusp }}^{0}\left(\bar{X}_{\mathrm{P}}^{o r}, \omega^{\kappa}\right) \rightarrow \mathrm{H}^{0}\left(\mathcal{J}_{\infty, 1} / \mathrm{P}, \omega^{\kappa}\right) .
$$

Il résulte du théorème précédent que la composé est d'image dense. On en déduit le corollaire :

Corollaire A.4. - Le module $\mathrm{H}_{\text {cusp }}^{0}\left(\bar{X}_{\mathrm{P}}, \omega^{\kappa}\right)^{\dagger}$ est dense dans le module $\mathrm{H}_{\text {cusp }}^{0}\left(\mathcal{J}_{\text {rig }, 1} / \mathrm{P}, \omega^{\kappa}\right)$.

On a de plus un isomorphisme

$$
e \mathrm{H}_{\text {cusp }}^{0}\left(\bar{X}_{\mathrm{P}}, \omega^{\kappa}\right)^{\dagger} \simeq e \mathrm{H}_{\text {cusp }}^{0}\left(\mathcal{T}_{\text {rig }, 1} / \mathrm{P}, \omega^{\kappa}\right) .
$$

\section{BIBLIOGRAPHIE}

[1] A. Abbès \& A. Mokrane - «Sous-groupes canoniques et cycles évanescents $p$-adiques pour les variétés abéliennes », Publ. Math. I.H.É.S. 99 (2004), p. 117-162.

[2] S. Bosch - «Lectures on formal and rigid geometry », prépublication http://wwwmath.uni-muenster.de/sfb/about/publ/heft378. pdf, 2005.

[3] G. Faltings \& C.-L. Chai - Degeneration of abelian varieties, Ergebn. Math. Grenzg., vol. 22, Springer, 1990.

[4] L. FARgues - «La filtration de Harder-Narasimhan des schémas en groupes finis et plats », J. reine angew. Math. 645 (2010), p. 1-39.

[5] A. Grothendieck \& J. Dieudonné - «Éléments de géométrie algébrique I, II, III, IV », Publ. Math. I.H.É.S. 4, 8, 11, 17, 20, 24, 28, 32 (19611967).

[6] H. Hida - « Iwasawa modules attached to congruences of cusp forms », Ann. Sci. École Norm. Sup. 19 (1986), p. 231-273. 
[7] _ , « Control theorems of coherent sheaves on Shimura varieties of PEL type », J. Inst. Math. Jussieu 1 (2002), p. 1-76.

[8] _ « $p$-adic automorphic forms on reductive groups », Astérisque 298 (2005), p. $147-254$.

[9] J. C. JANTZEn - Representations of algebraic groups, Pure and Applied Mathematics, vol. 131, Academic Press Inc., 1987.

[10] N. Jochnowitz - «A study of the local components of the Hecke algebra $\bmod l »$, Trans. Amer. Math. Soc. 270 (1982), p. 253-267.

[11] N. M. KATZ - « Serre-Tate local moduli», in Algebraic surfaces (Orsay, 1976-78), Lecture Notes in Math., vol. 868, Springer, 1981, p. 138-202.

[12] M. KISIN \& K. F. LAI - «Overconvergent Hilbert modular forms », Amer. J. Math. 127 (2005), p. 735-783.

[13] W. Messing - The crystals associated to Barsotti-Tate groups : with applications to abelian schemes, Lecture Notes in Math., vol. 264, Springer, 1972 .

[14] A. Mokrane \& J. Tilouine - « Cohomology of Siegel varieties with $p$-adic integral coefficients and applications », Astérisque 280 (2002), p. 195.

[15] D. Mumford - Abelian varieties, Tata Institute of Fundamental Research Studies in Mathematics, No. 5, Published for the Tata Institute of Fundamental Research, Bombay, 1970.

[16] F. OoRT - « Newton polygons and $p$-divisible groups : a conjecture by Grothendieck », Astérisque 298 (2005), p. 255-269.

[17] V. Pilloni - «Prolongement analytique sur les variétés de Siegel », Duke math. J. 157 (2011), p. 167-222.

[18] _ _ "Modularité, formes de Siegel et surfaces abéliennes », Journal für die reine und angewandte Mathematik 666 (2012), p. 35-82.

[19] I. SATAKE - « Theory of spherical functions on reductive algebraic groups over p-adic fields », Publ. Math. I.H.É.S. 18 (1963), p. 5-69.

[20] J-P. Serre - «Endomorphismes complètement continus des espaces de Banach p-adiques », Publ. Math. I.H.E.S. 12 (1962), p. 69-85.

[21] B. Stroh - « Compactifications des variétés de Siegel aux places de mauvaise réduction », thèse de doctorat, Université Henri Poincaré, Nancy, 2008.

[22] J. Tate \& F. OoRT - «Group schemes of prime order », Ann. Sci. École Norm. Sup. 3 (1970), p. 1-21. 